\title{
Method to Develop a Control System for a Stable and Guidable Hybrid Projectile
}

\author{
Joseph Close \\ West Virginia University
}

Follow this and additional works at: https://researchrepository.wvu.edu/etd

\section{Recommended Citation}

Close, Joseph, "Method to Develop a Control System for a Stable and Guidable Hybrid Projectile" (2014). Graduate Theses, Dissertations, and Problem Reports. 292.

https://researchrepository.wvu.edu/etd/292

This Thesis is protected by copyright and/or related rights. It has been brought to you by the The Research Repository @ WVU with permission from the rights-holder(s). You are free to use this Thesis in any way that is permitted by the copyright and related rights legislation that applies to your use. For other uses you must obtain permission from the rights-holder(s) directly, unless additional rights are indicated by a Creative Commons license in the record and/ or on the work itself. This Thesis has been accepted for inclusion in WVU Graduate Theses, Dissertations, and Problem Reports collection by an authorized administrator of The Research Repository @ WVU. For more information, please contact researchrepository@mail.wvu.edu. 


\title{
Method to Develop a Control System for a Stable and Guidable Hybrid Projectile
}

\author{
Joseph Close \\ Thesis submitted to the \\ College of Engineering and Mineral Resources \\ at West Virginia University \\ in partial fulfillment of the requirements for the degree of \\ Masters of Science \\ In \\ Mechanical Engineering
}

Jay Wilhelm, Ph.D., Chair

Marvin Cheng, Ph.D.

Gary Morris, Ph.D.

Department of Mechanical and Aerospace Engineering

Morgantown, West Virginia

2014

Keywords: Hybrid Projectile, guidance, stability

Copyright 2014 


\title{
Abstract \\ Method to Develop a Control System for a Stable and Guidable Hybrid Projectile Joseph Close
}

\begin{abstract}
A Hybrid Projectile (HP) is a munition that transforms into an unmanned aerial vehicle (UAV) after being launched from a tube. In many situations it is desirable for this type of projectile to change its point of impact and depart from its current ballistic trajectory similar to a UAV following a path. A method was created to utilize deflectable control surfaces in conjunction with a guidance system to ensure the HP was statically and dynamically stable and to maneuver the HP to a desired point of impact. Methods were devised to control heading and pitch using vertical and horizontal tail surfaces. Testing and tuning these control methods were done using the Six Degree of Freedom (6DoF) system in Simulink. A cruciform tail section was utilized so that the HP could be statically and dynamically stable. The simulation showed that the method devised was able to guide a $40 \mathrm{~mm}$ HP up to 6250 projectile diameters off of the line of fire and increase range by $25.8 \%$ while landing within 125 projectile diameters of the desired impact point.
\end{abstract}




\section{Acknowledgements}

I would like to thank my advisor Dr. Wilhelm for his guidance and knowledge through the process of finishing my Master's degree. I would also like to thank my committee members, Dr. Morris and Dr. Cheng, for providing suggestions to ensure my thesis was of highest quality. Also those who provided edits during early drafts of my thesis including Steve, Chris G., Chris M., Pat, Sheldon, Stephen and Celeste deserve acknowledgement for their contributions.

I could not have achieved my goal of a Master's degree without the support of my family. Without their encouragement and support I would have not succeeded.

Sincerely,

Joseph S. Close 


\section{Table of Contents}

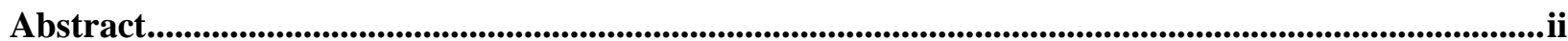

Acknowledgements ....................................................................................................................................iii

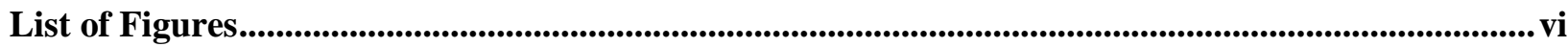

List of Tables ..............................................................................................................................viii

List of Symbols and Nomenclature.................................................................................................................ix

Chapter 1 Problem Statement............................................................................................. 1

$1.1 \quad$ Problem Description ................................................................................................. 1

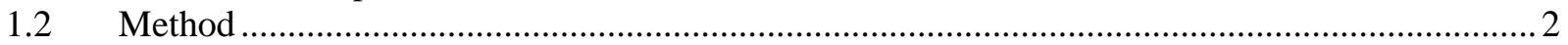

Chapter 2 Literature Review ................................................................................................................... 3

$2.1 \quad$ Specifications of $40 \mathrm{~mm}$ M781 ................................................................................... 3

2.2 Stability Analysis for Spinning and Non-spinning Projectiles.................................................... 5

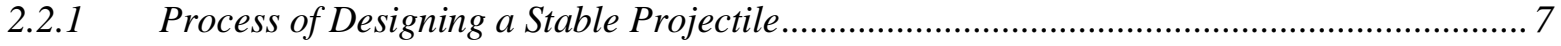

2.3 Modeling Flight using Equations of Motion.........................................................................

2.4 Six Degree of Freedom Simulation of M781 ........................................................................ 12

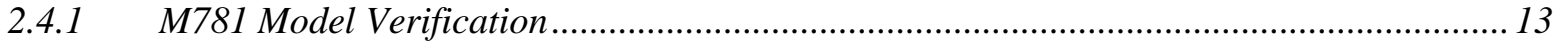

2.5 Investigate other HP's for Control System Methods ................................................................ 16

2.6 Control Methods for Aircraft Guidance................................................................................... 18

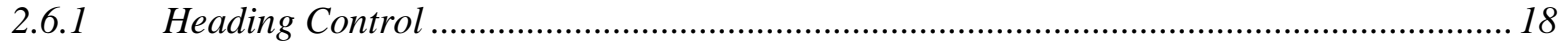

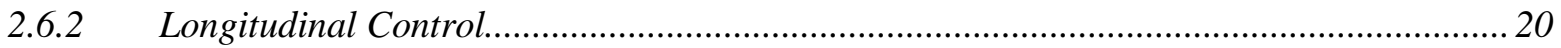

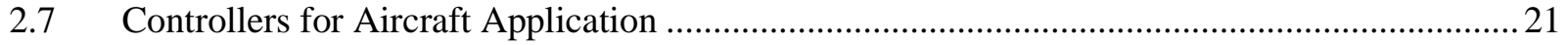

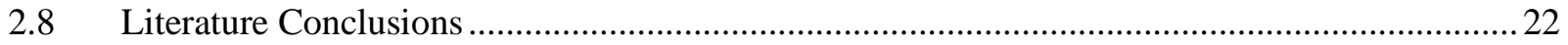

Chapter 3 Control and Stability for a 40 mm HP ................................................................................. 23

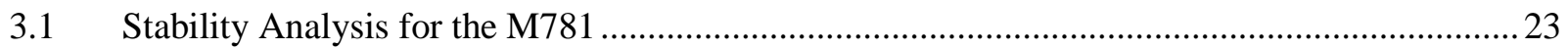

3.2 Adaptations Required for Stability of M781 to a $40 \mathrm{~mm} \mathrm{HP} \mathrm{...................................................2} 23$

3.2.1 Aerodynamic Coefficients Contribution of Tail Surfaces ................................................... 24

3.3 Verification of 6DoF model of a HP with Tails.........................................................................29

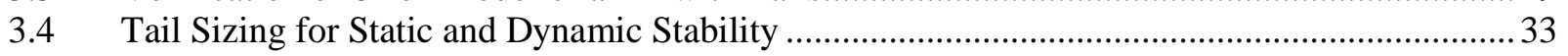

Chapter 4 Tail Control for Guidance................................................................................................ 37

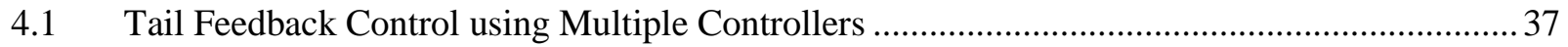

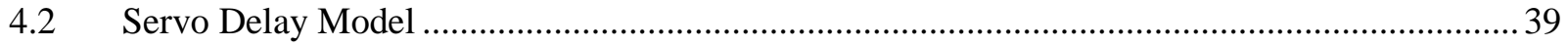

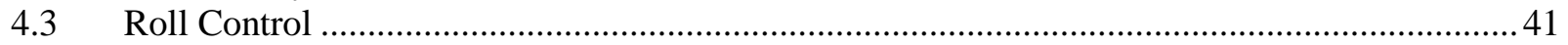

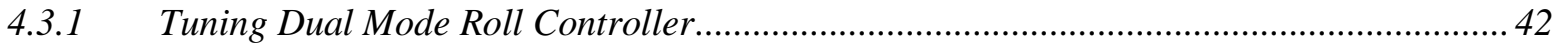

4.3.2 Determine Transition Point between Two Controllers ....................................................... 45

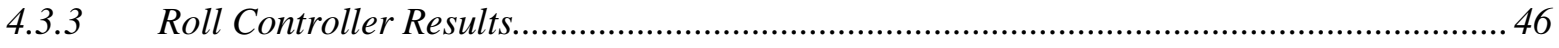

$4.4 \quad$ Heading Control....................................................................................................... 49

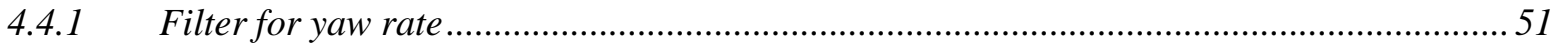

4.4.2 Tuning Heading Controller (Inner and Outer Loop).................................................... 52

4.4.3 Heading Controller Testing .............................................................................. 58

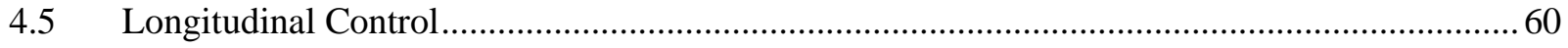

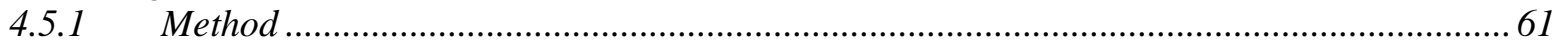


4.5.2 Find deflection magnitude, launch angle and pitch for tail deflection for max range........61

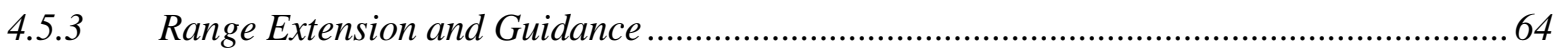

4.5.4 $\quad C_{L}, C_{D}$ and AoA when tails are deflected to zero degrees ............................................... 65

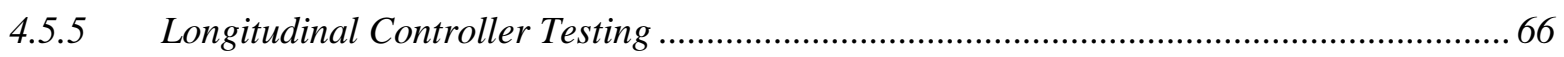

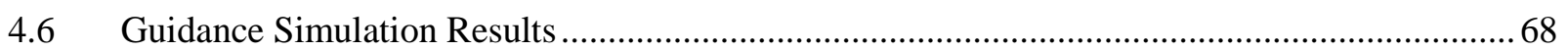

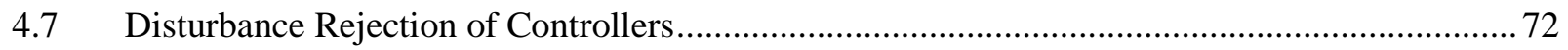

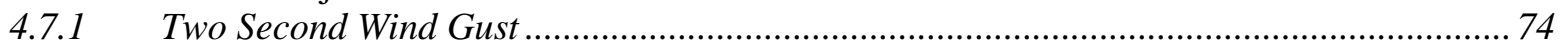

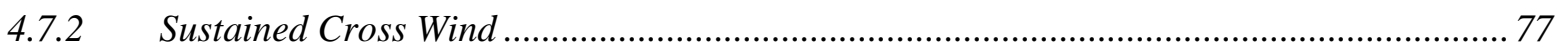

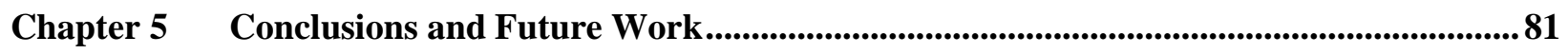

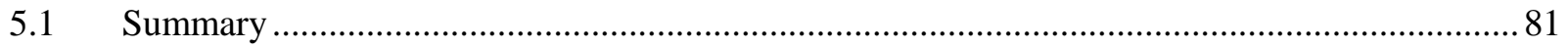

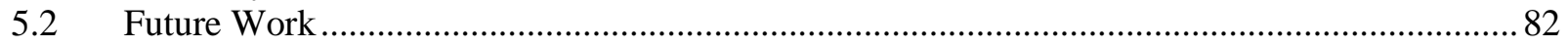

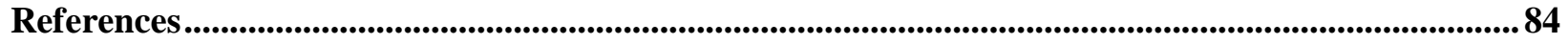

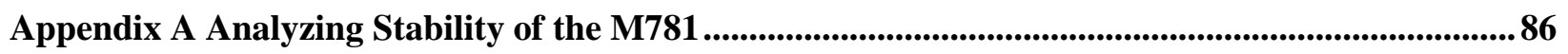

Appendix B Hand Calculations for Assumption of Tails Not Affecting Mass Properties................... 87

Appendix C CG and AC Calculation of Verification Model....................................................................... 89

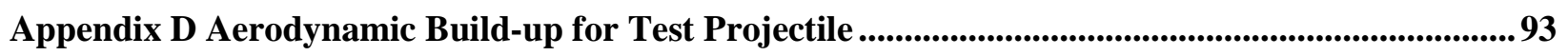

Appendix E Px4 Autopilot Setup.....................................................................................................................94

Appendix F Calculate Estimated Delta Distance Sub-system..................................................................95 


\section{List of Figures}

Figure 2-1: Body-fixed Coordinate System (Vogel 2012)...................................................................... 5

Figure 2-2: Flowchart to Check and Correct Stability of a Projectile...................................................... 8

Figure 2-3: Earth-Fixed and Body-Fixed Coordinate System ............................................................. 9

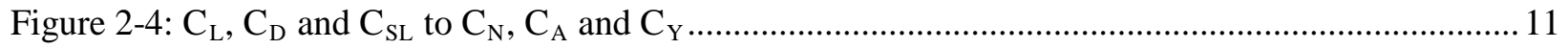

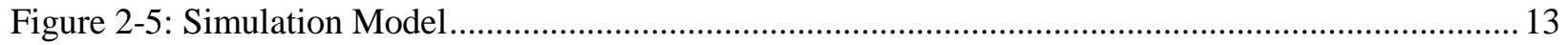

Figure 2-6: Simulation to Determine Max Range Launch Angle for M781 ............................................ 14

Figure 2-7: M781 Trajectory for 42 Deg. Launch Angle....................................................................... 14

Figure 2-8: Flight Path trajectories for 40 mm projectile from (Gkritzapis, et al. 2008).......................... 15

Figure 2-9: Flight Path Trajectories from Created Model .................................................................... 15

Figure 2-10: Stellar Exploration 40 mm Guided Munition (Exploration 2011) ......................................... 16

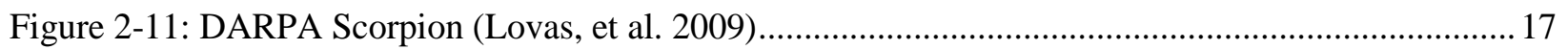

Figure 2-12: 155mm Puck Control Projectile (Fresconi, et al. 2011) ...................................................... 18

Figure 2-13: Outer Lateral Heading Angle Controller (Christiansen 2004) ............................................ 19

Figure 2-14: Inner Lateral Roll and Roll Rate Control (Christiansen 2004) ............................................ 19

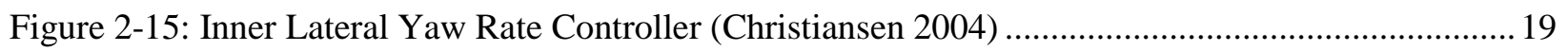

Figure 2-16: Outer Longitudinal Altitude Controller (Christiansen 2004) ..............................................20

Figure 2-17: Inner Longitudinal Airspeed Controller (Christiansen 2004) ............................................. 20

Figure 2-18: Inner Longitudinal Airspeed Controller (Christiansen 2004) ............................................ 21

Figure 3-1: Tail Deflections With Respect to Freestream Velocity .........................................................24

Figure 3-2: Full Projectile with Batteries (Front of Body) and Chip (Rear of Body)...............................29

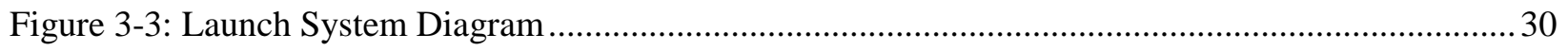

Figure 3-4: Test Projectile Launch, Zeroed from Starting Location.......................................................... 31

Figure 3-5: Simulation Results of Experimental Test.......................................................................... 32

Figure 3-6: Static Stability Examination with respect to Tail Sizing..................................................... 33

Figure 3-7: Dynamic Stability Examination with respect to Tail Sizing ................................................... 34

Figure 3-8: Effective Angle of Attack on tails from Roll Rate ............................................................ 35

Figure 3-9: Modified M781 with Four Tails....................................................................................... 36

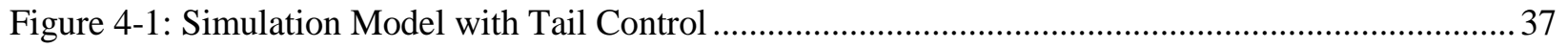

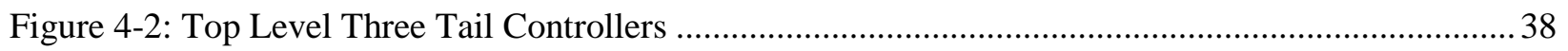

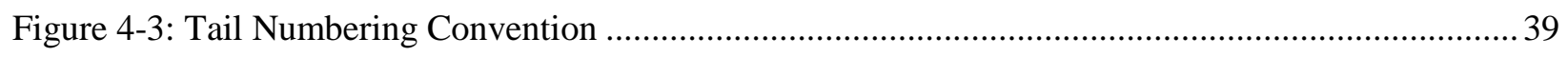

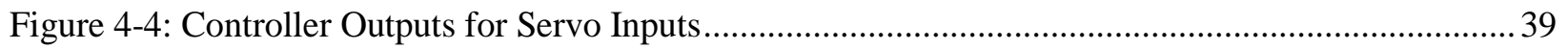

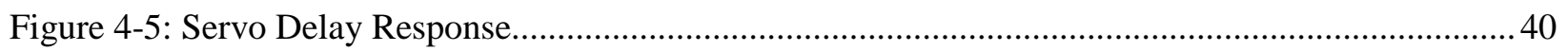

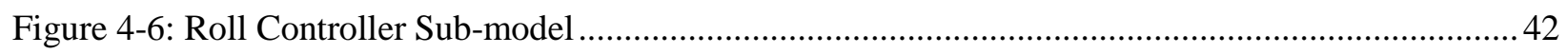

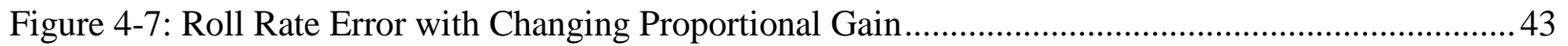

Figure 4-8: Position Error with Changing Derivative Gain .................................................................... 44

Figure 4-9: Roll Position Error with Changing Proportional Gain ......................................................... 44

Figure 4-10: Roll Position Error with Fine-tuning Derivative Gain ....................................................... 45

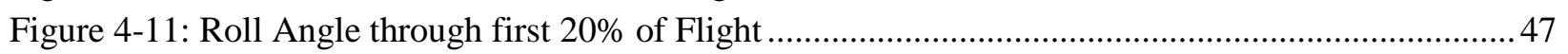

Figure 4-12: Roll Rate through first 20\% of Flight......................................................................... 47

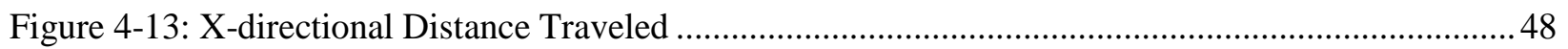




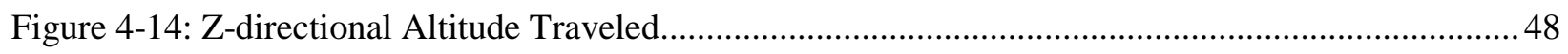

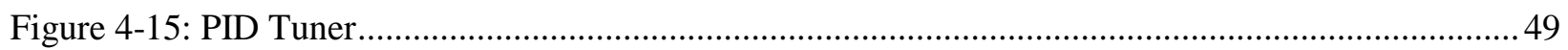

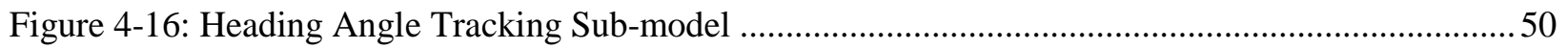

Figure 4-17: Desired Location Heading Tracking Sub-model................................................................. 50

Figure 4-18: Inner and Outer Loops of Heading Controller .................................................................. 51

Figure 4-19: Yaw Rate for Multiple First Order Filters........................................................................ 52

Figure 4-20: Setting Variables for Gain Values................................................................................... 53

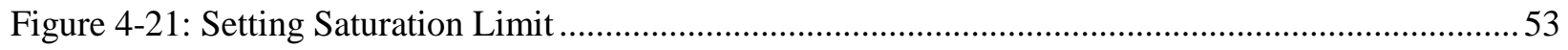

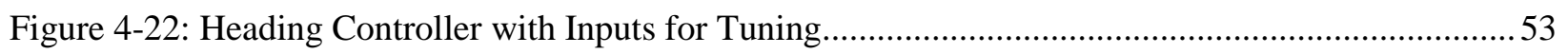

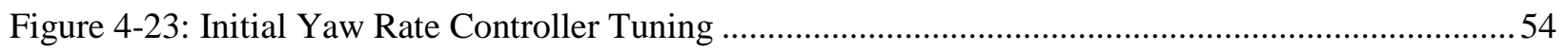

Figure 4-24: Yaw Rate Error and Vertical Tail Deflection Response from PID tune .............................. 55

Figure 4-25: Yaw Rate Response from 10 deg. Vertical Tail Deflection .................................................5

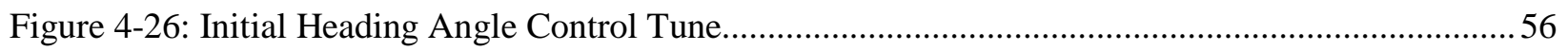

Figure 4-27: Heading Error and Yaw Rate Required from PID Tune .................................................... 57

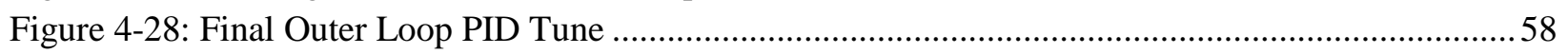

Figure 4-29: Simulation Test of Multiple Heading Angles ....................................................................59

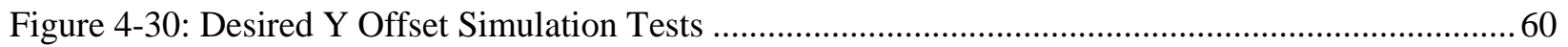

Figure 4-31: Distance/Altitude Longitudinal Controller Sub-system ......................................................6 61

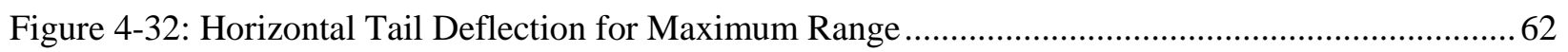

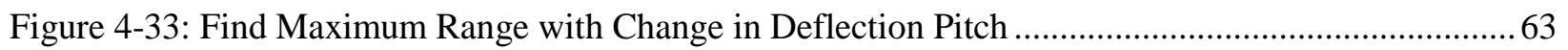

Figure 4-34: Find Maximum Range with Change in Deflection Pitch (Zoomed to Max Range)...............63

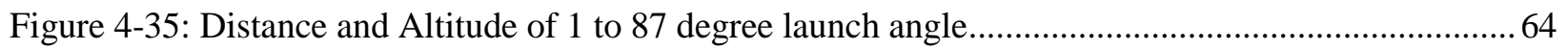

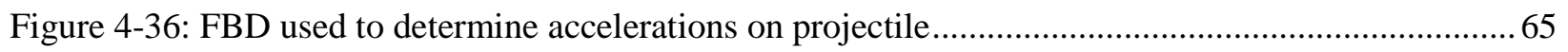

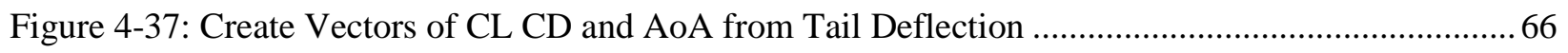

Figure 4-38: Longitudinal Target Distance $=122.5 \%$ Max Unguided Altitude=0\% Max Unguided .......... 67

Figure 4-39: Longitudinal Target Distance $=100 \%$ Max Unguided Altitude=50\% Max Unguided ...........67

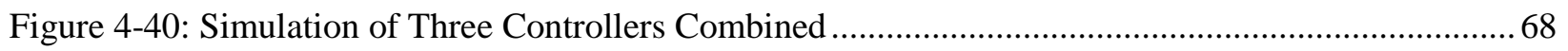

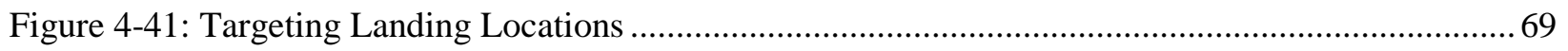

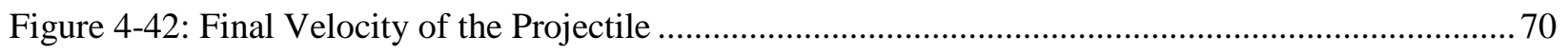

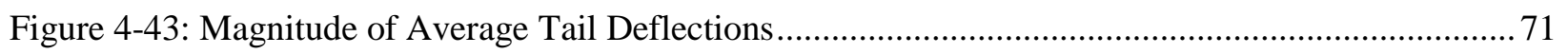

Figure 4-44: Ground Track of HP with Wind Gust to Desired Location 1............................................... 74

Figure 4-45: Ground Track of HP with Wind Gust to Desired Location 2............................................. 75

Figure 4-46: Ground Track of HP with Wind Gust to Desired Location 3.............................................. 76

Figure 4-47: Ground Track of HP with Sustained Wind to Desired Location 1....................................... 77

Figure 4-48: Ground Track of HP with Sustained Wind to Desired Location 2...................................... 78

Figure 4-49: Ground Track of HP with Sustained Wind to Desired Location 3........................................ 79

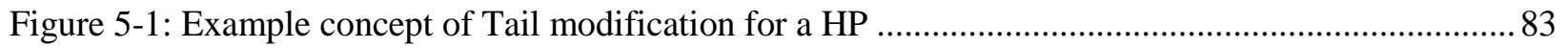

Figure C-1: Sketch to Determine Tail Dimensions (Barrowman n.d.) .................................................. 91 


\section{List of Tables}

Table 2-1: Standard M781 40 mm Practice Round Specifications ............................................................. 4

Table 2-2: Maximum Range and Altitude from Simulation and Literature Results ................................. 15

Table 3-1: Simulation Constants for the Experimental Projectile ........................................................... 32

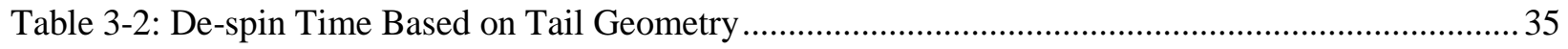

Table 4-1: Compilation of Gain Values from De-spin and Level Controller ............................................ 46

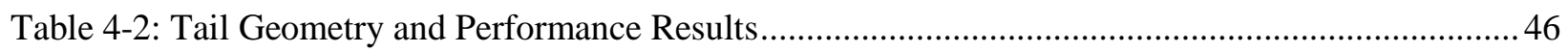

Table 4-3: Yaw Rate Response Characteristics from Multiple Cut-off Filters .......................................... 52

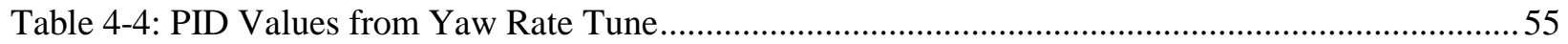

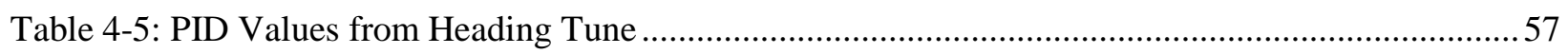

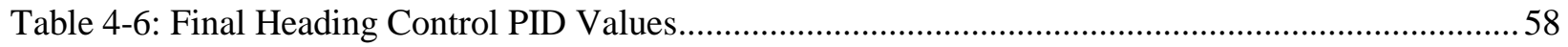

Table 4-7: Desired Target Locations for Y-Offset Control ..................................................................59

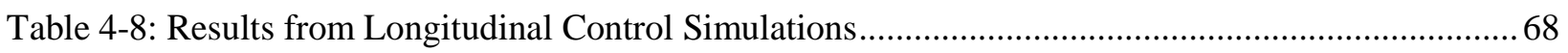

Table 4-9: Desired Impact Locations for Disturbance Rejection.............................................................. 73

Table 4-10: Impact offset due to Wind Gust to Desired Location 1 ........................................................ 74

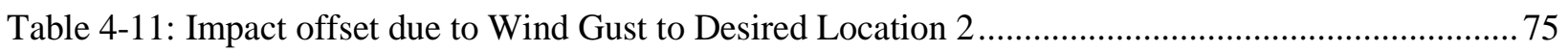

Table 4-12: Impact offset due to Wind Gust to Desired Location 3 ........................................................ 76

Table 4-13: Impact offset due to Sustained Wind to Desired Location 1 ................................................. 77

Table 4-14: Impact offset due to Sustained Wind to Desired Location 2 ............................................... 78

Table 4-15: Impact offset due to Sustained Wind to Desired Location 3.............................................. 79 


\section{List of Symbols and Nomenclature}

\begin{tabular}{|c|c|}
\hline Symbol & Description \\
\hline $6 D o F$ & Six Degrees of Freedom \\
\hline$A C$ & Aerodynamic center \\
\hline$A_{\text {ref }}$ & Reference area for the projectile \\
\hline$A_{\text {tails }}$ & Tail surface area \\
\hline$A R_{\text {tail }}$ & Tail aspect ratio \\
\hline$C_{A}$ & Axial force coefficient \\
\hline$C_{D}$ & Drag coefficient \\
\hline$C_{D_{\alpha}}$ & Change in drag coefficient with angle of attack \\
\hline$C_{D_{\text {Body }}}$ & Drag coefficient of the projectile body \\
\hline$C_{D_{i_{\text {Tails }}}}$ & Induced drag coefficient from the tails \\
\hline$C_{D_{o_{\text {Tails }}}}$ & Zero lift drag coefficient \\
\hline$C_{D_{\text {Tails }}}$ & Total drag coefficient from the tails \\
\hline$C F D$ & Computational fluid dynamics \\
\hline$C G$ & Center of gravity \\
\hline$C_{L}$ & Lift coefficient \\
\hline$C_{L_{o}}$ & Lift coefficient with no zero incidence \\
\hline$C_{L_{\alpha}}$ & Lift coefficient due to angle of attack \\
\hline$C_{L_{\alpha_{B o d y}}}$ & Lift coefficient due to angle of attack for the projectile body \\
\hline$C_{L_{\dot{\alpha}_{B o d y}}}$ & Lift coefficient due to rate of angle of attack for the projectile body \\
\hline$C_{L_{\dot{\alpha}_{H T}}}$ & Lift coefficient due to rate of angle of attack for the horizontal tails \\
\hline$C_{L_{\delta H T}}$ & Lift coefficient due to horizontal tail deflection \\
\hline$C_{\ell_{\text {Total }}}$ & Rolling moment coefficient \\
\hline$C_{\ell_{\text {deflected tails }}}$ & Rolling moment coefficient due to tails being deflected \\
\hline$C_{\ell_{\text {non-deflected tails }}}$ & Rolling moment coefficient due to rotational drag of tails \\
\hline$C_{\ell_{0}}$ & Rolling moment coefficient due to zero incidence and no deflections \\
\hline$C_{\ell_{p}}$ & Roll damping coefficient \\
\hline$C_{\ell_{\text {Tails }}}$ & Rolling moment coefficient due to tails \\
\hline$C_{\ell_{\alpha}}$ & Rolling moment coefficient due to change in angle of attack \\
\hline
\end{tabular}

Units

$m^{2}$

$m^{2}$

$-$ 


\begin{tabular}{|c|c|}
\hline$C_{\ell_{\beta}}$ & Rolling moment coefficient due to change of $\beta$ angle \\
\hline$C_{m_{\text {Total }}}$ & Pitching moment coefficient \\
\hline$C_{m_{H T}}$ & Pitching moment coefficient from the horizontal tails \\
\hline$C_{m_{o}}$ & Pitching moment coefficient change with zero incidence \\
\hline$C_{m_{p_{T a i ; s}}}$ & Pitching moment coefficient due to roll rate from the tails \\
\hline$C_{m_{q}}$ & Pitch damping coefficient \\
\hline$C_{m_{q_{H T}}}$ & Pitching moment coefficient from the horizontal tails \\
\hline$C_{m_{\alpha}}$ & Pitching moment coefficient due to angle of attack \\
\hline$C_{m_{\alpha_{B o d y}}}$ & Pitching moment coefficient due to angle of attack for the projectile body \\
\hline$C_{m_{\alpha_{c g}}}$ & Pitching moment coefficient due to angle of attack about the CG \\
\hline$C_{m_{\dot{\alpha}_{H T}}}$ & Pitching moment coefficient due to angle of attack for the horizontal tails \\
\hline$C_{m_{\delta H T}}$ & Pitching moment coefficient due to deflection of the horizontal tails \\
\hline$C_{N}$ & Normal force coefficient \\
\hline$C_{N_{\alpha}}$ & Normal force coefficient due to angle of attack \\
\hline$C_{n_{\text {Total }}}$ & Yawing moment coefficient \\
\hline$C_{n_{V T}}$ & Yawing moment coefficient from the vertical tails \\
\hline$C_{n_{o}}$ & Yawing moment coefficient change with zero incidence \\
\hline$C_{n_{p_{T a i ; s}}}$ & Yawing moment coefficient due to roll rate from the tails \\
\hline$C_{n_{r}}$ & Yaw damping coefficient \\
\hline$C_{n_{r_{V T}}}$ & Yawing moment coefficient from the vertical tails \\
\hline$C_{n_{\beta}}$ & Yawing moment coefficient due to $\beta$ angle \\
\hline$C_{n_{\beta_{B o d y}}}$ & Yawing moment coefficient due to $\beta$ angle for the projectile body \\
\hline$C_{n_{\dot{\beta}_{V T}}}$ & Yawing moment coefficient due to $\beta$ angle for the vertical tails \\
\hline$C_{n_{\delta V T}}$ & Yawing moment coefficient due to deflection of the vertical tails \\
\hline$c_{r}$ & Root chord length for the tails \\
\hline$C_{S L}$ & Side lift coefficient \\
\hline$C_{S L_{o}}$ & Side lift coefficient with no zero incidence \\
\hline$C_{S L_{\beta}}$ & Side lift coefficient due to $\beta$ angle \\
\hline$C_{S L_{\beta_{B o d y}}}$ & Side lift coefficient due to $\beta$ angle for the projectile body \\
\hline$C_{S L_{\dot{\beta}_{B o d y}}}$ & Side lift coefficient due to rate of $\beta$ angle for the projectile body \\
\hline
\end{tabular}




\begin{tabular}{|c|c|c|}
\hline$C_{S L_{\dot{\beta}_{V T}}}$ & Side lift coefficient due to rate of $\beta$ angle for the vertical tails & - \\
\hline$C_{S L_{\delta V T}}$ & Side lift coefficient due to vertical tail deflection & - \\
\hline$c_{t}$ & Tip chord length for the tails & $m$ \\
\hline$C_{Y}$ & Side force coefficient & - \\
\hline$d$ & Projectile diameter & $m$ \\
\hline$D$ & Drag force & $N$ \\
\hline$D_{\text {inner }}$ heading & Inner loop heading PID controller derivative gain & - \\
\hline$D_{\text {outer }_{\text {heading }}}$ & Outer loop heading PID controller derivative gain & - \\
\hline$d_{p o s}$ & Roll-leveling PID controller derivative gain & - \\
\hline$d_{\text {roll }}$ & De-spin PID controller derivative gain & - \\
\hline$F$ & Force & $N$ \\
\hline$F_{x}$ & Force in the $\mathrm{x}$-direction & $N$ \\
\hline$F_{y}$ & Force in the y-direction & $N$ \\
\hline$F_{z}$ & Force in the z-direction & $N$ \\
\hline$g$ & Gravity & $\mathrm{m} / \mathrm{s}^{2}$ \\
\hline$H$ & Non-dimensional stability term & - \\
\hline$H P$ & Hybrid projectile & - \\
\hline$I_{\text {inner }}$ heading & Inner loop heading PID controller integral gain & - \\
\hline$I_{\text {outer }}$ heading & Outer loop heading PID controller integral gain & - \\
\hline$I_{x x}$ & Moment of inertia about the $\mathrm{x}$-axis & $k g m^{2}$ \\
\hline$I_{x z}$ & Product of inertia about the xz-axis & $k g m^{2}$ \\
\hline$I_{y y}$ & Moment of inertia about the $y$-axis & $k g m^{2}$ \\
\hline$I_{z z}$ & Moment of inertia about the z-axis & $k g m^{2}$ \\
\hline$i_{\text {pos }}$ & Roll-leveling PID controller integral gain & - \\
\hline$i_{\text {roll }}$ & De-spin PID controller integral gain & - \\
\hline$L$ & Lift force & $N$ \\
\hline$\ell$ & Rolling moment & $N m$ \\
\hline$\ell_{r e f}$ & Projectiles reference length & $m$ \\
\hline$\ell_{\text {Tail }}$ & Length from reference point to the $\mathrm{AC}$ of the tails & $m$ \\
\hline$M$ & Static stability factor & - \\
\hline$M_{p}$ & Mass of the Projectile & $\mathrm{kg}$ \\
\hline$m$ & Pitching moment & $N m$ \\
\hline
\end{tabular}




\begin{tabular}{|c|c|}
\hline$N$ & Number of tails \\
\hline$N$ & PID filter coefficient \\
\hline$N_{\text {inner }}$ heading & Inner loop heading PID controller filter coefficient \\
\hline$N_{\text {outer }}$ heading & Outer loop heading PID controller filter coefficient \\
\hline$n$ & Yawing Moment \\
\hline off $f_{\text {NoWind }}$ & Impact offset from desired with no wind \\
\hline Of $f_{\text {Wind }}$ & Impact offset from desired with wind \\
\hline$P$ & Roll rate factor for stability \\
\hline$P_{\text {inner }} r_{\text {heading }}$ & Inner loop heading PID controller proportional gain \\
\hline$P_{\text {outer }}$ heading & Outer loop heading PID controller proportional gain \\
\hline$P I D$ & Proportional, derivative and integral control \\
\hline$p$ & Roll rate \\
\hline$\dot{p}$ & Rolling acceleration \\
\hline$p_{\text {min }}$ & Minimum roll rate for stability \\
\hline$p_{\text {pos }}$ & Roll-leveling PID controller proportional gain \\
\hline$p_{\text {roll }}$ & De-spin PID controller proportional gain \\
\hline$q$ & Pitch rate \\
\hline$\dot{p}$ & Pitching acceleration \\
\hline $\bar{q}$ & Dynamic pressure \\
\hline$R e$ & Reynolds number \\
\hline$r$ & Yaw rate \\
\hline$\dot{r}$ & Yawing acceleration \\
\hline$r_{t}$ & Moment arm from the $\mathrm{x}$-axis for the tail \\
\hline $\bar{r}_{x}$ & Radius of gyration about the $\mathrm{x}$-axis \\
\hline $\bar{r}_{y}$ & Radius of gyration about the $y$-axis \\
\hline$S_{d}$ & Dynamic stability criteria \\
\hline$S_{g}$ & Gyroscopic stability criteria \\
\hline$S L$ & Side lift force \\
\hline$S M$ & Static margin \\
\hline$s$ & Span of each tail \\
\hline$T$ & Non-dimensional stability term \\
\hline$t$ & Time \\
\hline$U A V$ & Unmanned Aerial Vehicle \\
\hline
\end{tabular}

$\mathrm{deg} / \mathrm{s}$

$\operatorname{deg} / \mathrm{s}^{2}$

$\mathrm{deg} / \mathrm{s}$

$\mathrm{deg} / \mathrm{s}$

$\operatorname{deg} / \mathrm{s}^{2}$

$\mathrm{N} / \mathrm{m}^{2}$

$\mathrm{deg} / \mathrm{s}$

$\operatorname{deg} / \mathrm{s}^{2}$

m

m

$m$

N

$m$

s 


\begin{tabular}{|c|c|c|}
\hline$u$ & Velocity in the $\mathrm{x}$-direction for the body & $\mathrm{m} / \mathrm{s}$ \\
\hline$\dot{u}$ & Acceleration in the x-direction for the body & $\mathrm{m} / \mathrm{s}^{2}$ \\
\hline$V$ & Projectile total velocity & $\mathrm{m} / \mathrm{s}$ \\
\hline$V_{e}$ & Projectile's velocity with respect to the ground & $\mathrm{m} / \mathrm{s}$ \\
\hline$V_{\infty}$ & Freestream velocity & $\mathrm{m} / \mathrm{s}$ \\
\hline$v$ & Velocity in the y-direction for the body & $\mathrm{m} / \mathrm{s}$ \\
\hline$\dot{v}$ & Acceleration in the y-direction for the body & $m / s^{2}$ \\
\hline$W V U$ & West Virginia University & - \\
\hline$w$ & Velocity in the z-direction for the body & $\mathrm{m} / \mathrm{s}$ \\
\hline$\dot{w}$ & Acceleration in the z-direction for the body & $\mathrm{m} / \mathrm{s}^{2}$ \\
\hline$X_{A C}$ & Length from nose of projectile to its AC & $m$ \\
\hline$X_{b}$ & Body oriented $\mathrm{x}$-axis & - \\
\hline$X_{C G}$ & Length from nose of projectile to its CG & $m$ \\
\hline$X_{e}$ & Earth oriented $\mathrm{x}$-axis & - \\
\hline$X_{R e f}$ & Length from nose of projectile to its reference location & $m$ \\
\hline$X_{S M}$ & Length of static margin & $m$ \\
\hline$x$ & Downrange position of the projectile & $m$ \\
\hline$\dot{x}_{e}$ & Downrange velocity of the projectile with respect to the earth & $\mathrm{m} / \mathrm{s}$ \\
\hline$Y_{b}$ & Body oriented y-axis & - \\
\hline$Y_{e}$ & Earth oriented y-axis & - \\
\hline$y$ & Off line of fire position of the projectile & $m$ \\
\hline$\dot{y}_{e}$ & Off line of fire velocity of the projectile with respect to the earth & $m / s$ \\
\hline$Z_{b}$ & Body oriented z-axis & - \\
\hline$Z_{e}$ & Earth oriented z-axis & - \\
\hline$z$ & Altitude of the projectile & $m$ \\
\hline$\dot{z}_{e}$ & Change in altitude of the projectile with respect to the earth & $\mathrm{m} / \mathrm{s}$ \\
\hline$\alpha$ & Angle of attack & $\operatorname{deg}$ \\
\hline$\dot{a}$ & Rate of change in angle of attack & $\mathrm{deg} / \mathrm{s}$ \\
\hline$\beta$ & Projectiles y-direction deviation from freestream & $\operatorname{deg}$ \\
\hline$\dot{\beta}$ & Rate of change in projectiles y-direction deviation from freestream & $\mathrm{deg} / \mathrm{s}$ \\
\hline$\Delta \varphi$ & Difference in roll angle from roll level & $\operatorname{deg}$ \\
\hline$\delta$ & Deflections used for roll & $\operatorname{deg}$ \\
\hline$\delta_{H T}$ & Deflection of the horizontal tails & $\operatorname{deg}$ \\
\hline$\delta_{V T}$ & Deflection of the vertical tails & deg \\
\hline
\end{tabular}


$\eta \quad$ Tail efficiency

$\theta \quad$ Pitch angle

deg

Rate of change in pitch angle

deg

Yaw of repose

Air density

$\mathrm{kg} / \mathrm{m}^{3}$

Roll angle

deg

Rate of change in roll angle

deg

Yaw angle

deg

Rate of change in yaw angle

deg

Cutoff frequency for filter

$\mathrm{Hz}$ 


\section{Problem Statement}

\subsection{Problem Description}

A projectile is an object put into motion by an external force then continues on a path by its own inertia, affected only by aerodynamic forces and gravity. Traditionally, projectiles have not had the ability to deviate from their ballistic trajectory after being launched. If a projectile could transform and be controlled, it could act like an Unmanned Aerial Vehicle (UAV) yet still be able to be launched ballistically. A Hybrid Projectile (HP) was investigated at West Virginia University (WVU) to develop a design to alter a projectile into a UAV after being tube launched. The HP developed in this study considered range extension, guidance, and maneuvering along a flight path or to a point of impact.

Guidance and range extension could be important additions to any currently designed spinstabilized projectile. Most spin-stabilized projectiles must remain spinning after being launched because they are statically unstable since the projectile's Aerodynamic Center (AC) is forward of the Center of Gravity (CG). However, it was desired that a HP launched from a rifled barrel be non-spinning during the guidance portion of the flight to utilize body lift for range extension and simplify control laws. When a

projectile is not spinning, it must be statically and dynamically stable to be maneuvered. Being statically stable means that when the projectile is disturbed from a steady state orientation, it naturally returns to its equilibrium state. The HP considered also needed be dynamically stable such that the oscillations back to its equilibrium state were properly damped. Adequately sized control surfaces were needed to move the AC aft of the CG, to provide stability. Problem Statement: A design methodology was sought, which utilizes deflectable control surfaces in conjunction with a guidance system to ensure the HP remains statically and dynamically stable once de-spun and to maneuver the HP to a desired point of impact while using the body lift to extend range.

Guiding a projectile to a desired point of impact requires modifications to a traditionally designed round for control and stability. Due to these goals, a control system must be designed to de-spin and roll level a statically and dynamically stable projectile while maneuvering it to a desired point of impact. A method consisting of proportional, integral and derivative (PID) feedback control was considered and applied to an existing projectile, the M781. The M781 is a $40 \mathrm{~mm}$ projectile that is used as a practice round. This projectile is unguidable and the trajectory can only be changed, pre-launch, by moving the tube elevation and azimuth. Because of its availability and small size the M781 was chosen to be adapted. 


\subsection{Method}

A method was created to size and locate control surfaces and develop a control system for a 40 mm HP based on a M781. The first step in the process of adapting a $40 \mathrm{~mm}$ muntion into a HP was to conduct a literature review for the aerodynamic data and specifications for the baseline $40 \mathrm{~mm}$ projectile. Stability was then analyzed to determine if control surfaces would be required if the HP was not spinning. From the stability analysis, the control surface type was determined and surface area was calculated due to de-spin and stability specifications. In this case, tails were used because of their tendency to pitch the projectile down as angle of attack increases to add static stability. A build-up of aerodynamic coefficients was then created to add the contribution of the tails to the body so that the forces and moments could be calculated in the equations of motion. Aerodynamic coefficients and the projectile's geometry were used to model the flight of the projectile in Simulink. With control surfaces applied to the projectile, a roll controller was created to deflect the local angle of attack of the tail surfaces to de-spin and roll-level the projectile. Control methods were devised for a roll-leveled projectile to change its heading and pitch, maneuvering the HP to a desired point of impact. Vertical tail surfaces were used to change the HP's yaw angle to make left and right maneuvers off the line of fire. The horizontal tail surfaces were used to increase the angle of attack in order to use the body as a lifting surface, extending range. Horizontal tails were then deflected back to zero degrees so that its estimated ballistic trajectory would reach the desired distance and altitude. Testing and tuning of control system were done using the aerospace and controls toolbox of MATLAB's Simulink (Matlab 2014). An impact grid of achievable impact locations was created to show the area in which guidance was achievable to within 125 projectile diameters of a desired location. Disturbance rejection of the controllers was predicted by simulating guidance results to examine if the HP's impact point would be affected due to a two second side gust or sustained cross wind. 


\section{Chapter 2 Literature Review}

Designing a guidable hybrid projectile required literature research before the process was commenced. First, a literature review was required to determine the aerodynamic coefficients, geometry, mass properties and launch conditions for the M781 $40 \mathrm{~mm}$ projectile. Equations to analyze stability were found so that it could be determined if the M781 was gyroscopically, statically and dynamically stable. Equations of motions were then researched and used to model the flight of the projectile using the specifications collected for the M781. The projectile was modeled using the aerospace toolbox in Simulink and results were compared to literature data to verify accuracy. Other Hybrid Projectiles were then investigated for information on types of control surfaces which could be used for maneuvering the HP. Controller method and types also needed to be researched to determine feedback control methods that were commonly used for guiding UAVs.

\subsection{Specifications of $40 \mathrm{~mm} M 781$}

The projectile in this study that was chosen to be adapted to a HP was the M781 $40 \mathrm{~mm}$ practice round. This projectile was chosen because of its well-known performance. Aerodynamic coefficients, geometry and launch conditions for the basic M781 $40 \mathrm{~mm}$ projectile used in this study were found in literature and compiled in Table 2-1. The launch velocity of the $40 \mathrm{~mm}$ projectile was found to be 76 meters per second and the initial roll rate was to be $62.5 \mathrm{~Hz}$ and the mass $\left(\mathrm{M}_{\mathrm{p}}\right)$ of the $\mathrm{M} 781$ was found to be $0.205 \mathrm{~kg}$ (Cooke 2010). The moment of inertia around each of the three axes for the M781 being investigated was found through literature and compiled in Table 2-1, as well (Martinez, Bruno and Kelly 2013).

The drag coefficient was determined as an average of two separate literature sources to be 0.2775 (Fresoni and Plostins 2011) (Gkritzapis, et al. 2008). Similarly for the lift coefficient due to change in angle of attack $\left(C_{L_{\alpha}}\right)$, the average of both sources was used and calculated to be 0.0378 per degree of angle of attack change (Fresoni and Plostins 2011) (Gkritzapis, et al. 2008). Between the two sources, there was a $1 \%$ difference in $C_{D}$ and $10 \%$ difference in $C_{L_{\alpha}}$. Due to the M781 being an axisymmetric projectile the side lift coefficient due change in $\beta\left(C_{S L_{\beta}}\right)$ was the same as $C_{L_{\alpha}}$.

From Computational Fluid Dynamics (CFD), the pitching moment coefficient due to change in angle of attack $\left(C_{m_{\alpha}}\right)$ was found as -0.0273 (Fresoni and Plostins 2011). However $C_{m_{\alpha}}$ used in the current study was 0.0273 . The sign was reversed for $C_{m_{\alpha}}$ in this study because the static margin (SM), distance from the aerodynamic center (AC) to center of gravity (CG), being used in their study was positive since the CG was aft of the AC. The reference location $\left(\mathrm{X}_{\text {ref }}\right)$ in this study was at the nose so the calculated the static margin, $\mathrm{X}_{\mathrm{SM}}=\mathrm{X}_{\mathrm{ref}}-\mathrm{X}_{\mathrm{AC}}$ was determined to be negaitive. Static margin was used to calculate the 
pitching moment coefficient as $\mathrm{C}_{\mathrm{m}_{\alpha}}=\mathrm{C}_{\mathrm{L}_{\alpha}}\left(\mathrm{X}_{\mathrm{SM}}\right)$, where the pitching moment was found to be negative. Due to symmetry of the body the yawing moment coefficient due to the change in $\beta\left(\mathrm{C}_{\mathrm{n}_{\beta}}\right)$ was also determined to be -0.0273 . Pitch and yaw damping coefficient $\left(C_{m_{q}}\right.$ and $C_{n_{r}}$, respectively) due to pitch rate (q) and yaw rate (r) were found to be -0.1 (Dupuis 2007). In (Lyon 1997) the Magnus force coefficient $\left(C_{m_{p_{\alpha}}}\right.$ ) for the M781 was found to be 0.1325 .

Table 2-1: Standard M781 40 mm Practice Round Specifications

\begin{tabular}{|c|c|c|}
\hline Constant & Value & Source \\
\hline$C_{D}$ & 0.2775 & $\begin{array}{l}\text { (Fresoni and Plostins 2011), } \\
\text { (Gkritzapis, et al. 2008) }\end{array}$ \\
\hline$C_{L_{\alpha}}$ and $C_{S L_{\beta}}$ & $\begin{array}{c}2.166 \text { (per rad) } \\
0.0378 \text { (per deg.) }\end{array}$ & $\begin{array}{l}\text { (Fresoni and Plostins 2011), } \\
\text { (Gkritzapis, et al. 2008) }\end{array}$ \\
\hline$C_{m_{\alpha_{C G}}}$ and $C_{n_{\beta_{C G}}}$ & $\begin{array}{c}1.564 \text { (per rad) } \\
0.0273 \text { (per deg.) }\end{array}$ & (Fresoni and Plostins 2011) \\
\hline$C_{m_{p_{\alpha}}}$ & $\begin{array}{l}0.1325 \text { (per rad) } \\
0.0023 \text { (per deg.) }\end{array}$ & (Lyon 1997) \\
\hline$C_{m_{q}}$ and $C_{n_{r}}$ & $\begin{array}{l}-5.73 \text { (per rad) } \\
-0.1 \text { (per deg.) }\end{array}$ & (Dupuis 2007) \\
\hline$d$ & $0.04 \mathrm{~m}$ & \\
\hline$I_{x x}$ & $5.4412 \times 10^{-5} \mathrm{~kg} \mathrm{~m}^{2}$ & (Martinez, Bruno and Kelly 2013) \\
\hline$I_{y y}$ and $I_{z z}$ & $1.19215 \times 10^{-4} \mathrm{~kg} \mathrm{~m}^{2}$ & (Martinez, Bruno and Kelly 2013) \\
\hline$M_{p}$ & $0.205 \mathrm{~kg}$ & (Cooke 2010) \\
\hline$p$ & $62.5 \mathrm{~Hz}$ & (Cooke 2010) \\
\hline$A_{\text {ref }}$ & $0.00126 \mathrm{~m}^{2}$ & \\
\hline$V$ & $76 \mathrm{~m} / \mathrm{s}$ & (Cooke 2010) \\
\hline$X_{A C}$ & $0.0142 \mathrm{~m}$ & (Martinez, Bruno and Kelly 2013) \\
\hline$X_{C G}$ & $0.0864 \mathrm{~m}$ & (Dupuis 2007) \\
\hline
\end{tabular}




\begin{tabular}{|l|l|l|}
\hline$\rho$ & $1.2 \mathrm{~kg} / \mathrm{m}^{3}$ & \\
\hline
\end{tabular}

\subsection{Stability Analysis for Spinning and Non-spinning Projectiles}

Research was conducted to determine if stability of a projectile could be analytically calculated. Equations were found in (Milinovic, et al. 2012), (Wilson 2007) and (Carlucci and Jacobson 2008) that used the projectile's geometry and aerodynamic coefficients to analyze the stability of a non-spinning or spinning projectile. The stability criterion for a projectile differs depending if the projectile is spinning or not. A spinning projectile must be gyroscopically and dynamically stable, and if the projectile is not spinning, it must be statically and dynamically stable. Criteria found in literature were used to estimate stability of the projectile. Figure 2-1 shows the orientation of the projectile and was necessary in order to determine the moments of inertia about each axis (Vogel 2012). In Figure 2-1 (Vogel 2012) used the axis labels 1, 2 and 3 to describe the earth referenced coordinate system, x, $\mathrm{y}$ and $\mathrm{z}$, respectively.

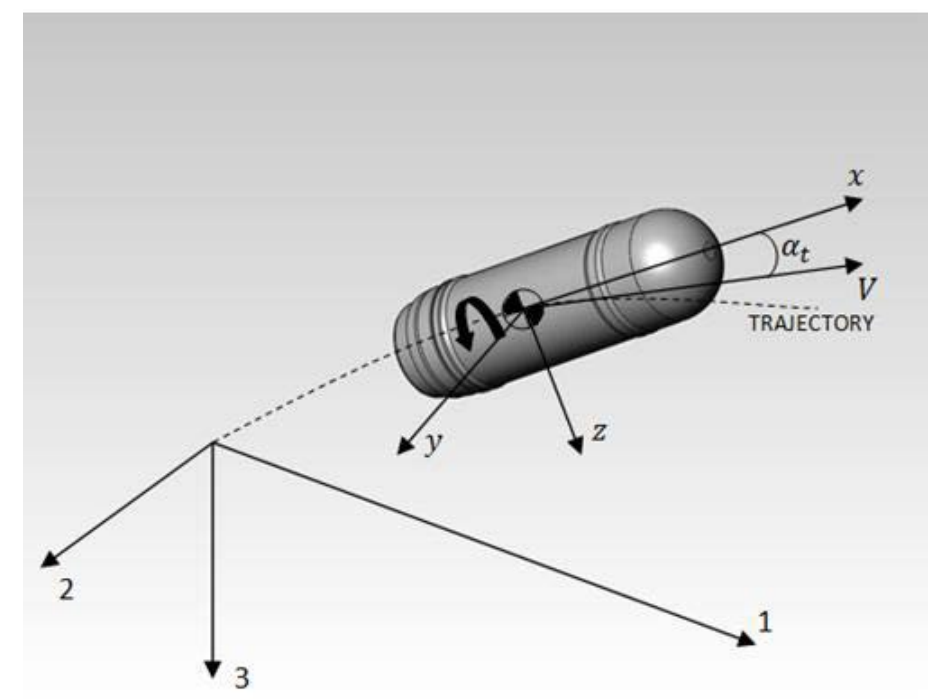

Figure 2-1: Body-fixed Coordinate System (Vogel 2012)

The gyroscopic stability criterion $\left(\mathrm{S}_{\mathrm{g}}\right)$, defined in equation (2-1), was used to calculate if a projectile would be gyroscopically stable due do its mass properties, motion and aerodynamics. If $\mathrm{S}_{\mathrm{g}}$ was greater than ' 1 ' then the projectile was estimated to be gyroscopically stable and unstable if below one (Milinovic, et al. 2012) (Wilson 2007) (Carlucci and Jacobson 2008). Similar to a spinning top, if the spin rate is high enough the projectile will remain oriented with the freestream velocity. After looking at equation (2-1) there is a minimum roll rate $\left(p_{\min }\right)$ where $S_{g}$ falls below ' 1 '. The value of $p_{\min }$ could be considered the minimum roll rate for gyroscopic stability for a particular projectile with known moments of inertia, aerodynamics and dimensions. This roll rate of the projectile could be adjusted by adding canted fins to the projectile or changing the rifling in the barrel. A projectile with a constant moment of inertia about the $x$-axis $\left(I_{x x}\right)$, moment of inertia about the $y$-axis $\left(I_{y y}\right)$, reference area $\left(A_{\text {ref }}\right)$, pitching 
moment coefficient with respect to $C G\left(\mathrm{C}_{\mathrm{maCG}}\right)$ and diameter $(\mathrm{d})$ at a specific flight air density $(\rho)$ and velocity (V) could only have its roll rate (p) adjusted to ensure a projectile was gyroscopically stable.

$$
S_{g}=\frac{I_{x x}^{2} p^{2}}{2 \rho I_{y y} A_{r e f} d V^{2} C_{m_{\alpha}}}
$$

A projectile may be gyroscopically stable according to the $\mathrm{S}_{\mathrm{g}}$ calculated, but it is also important that the projectile was dynamically stable. Dynamically stable means that the oscillatory motion of the projectile naturally returning to steady state is properly damped. In (Raymer 1999), the dynamic stability for an aircraft refers to the balance of inertial and damping forces resisting the change in body angle rates. The damping forces are aerodynamic forces that are proportional to the pitch, roll and yaw rates (Raymer 1999). A dynamically stable projectile is similar to an overdamped or critically damped mechanical systems where the pitch, roll and yaw angles oscillate to a steady state equilibrium value. A projectile is determined to be dynamically stable if the inequality of equation (2-7) was true. Equation (2-7) required that the dynamic stability criteria $\left(S_{d}\right)$, equation (2-6) and $S_{g}$, equation (2-1) be known.

Calculating those two values was done by solving equations (2-2) through (2-5). Where equation (2-2) was used to solve for the non-dimensional radius of gyration about the $\mathrm{x}$-axis $\left(\overline{\mathrm{r}}_{\mathrm{x}}\right)$ and equation (2-3) solves the non-dimensional radius of gyration about the y-axis ( $\left.\overline{\mathrm{r}}_{\mathrm{y}}\right)$ (Wilson 2007). In equations (2-4) and (2-5) the values $T$ and $H$ were used as a non-dimensional terms to consolidate equation (2-6) where $C_{D}$ was the drag coefficient and $\mathrm{C}_{\mathrm{mq}}$ was the pitch damping coefficient. Equation (2-6) was used to calculate $\mathrm{S}_{\mathrm{d}} \cdot \mathrm{C}_{\mathrm{L}_{\alpha}}$ was the lift coefficient with change in angle of attack and $\mathrm{C}_{\mathrm{m}_{\mathrm{p}_{\alpha}}}$ was the pitching moment coefficient due the projectile rolling and being at an angle of attack.

$$
\begin{gathered}
\bar{r}_{x}=\sqrt{\frac{I_{x x}}{M_{p} d^{2}}} \\
\bar{r}_{y}=\sqrt{\frac{I_{y y}}{M_{p} d^{2}}} \\
T=\left(\frac{\rho A_{r e f} d^{3}}{2 I_{y y}}\right)\left(C_{L_{\alpha}}+\bar{r}_{x}^{-2} C_{m_{p_{\alpha}}}\right) \\
H=\left(\frac{\rho A_{r e f} d}{2 M_{p}}\right)\left(C_{L_{\alpha}}-C_{D}-\bar{r}_{y}^{-2} C_{m_{q}}\right) \\
S_{d}=\frac{2 T}{H} \\
\frac{1}{S_{g}}>S_{d}\left(2-S_{d}\right)
\end{gathered}
$$


For a spinning projectile, the Magnus force component also causes the projectile to move left or right off the line of fire, called yaw of repose $\left(\xi_{g}\right)$ (Milinovic, et al. 2012) (Wilson 2007) (Jie, et al. 2013). In equation (2-8), the Magnus direction of drift is estimated due to the sign of the value inside the parenthesis. If the static stability criterion (M) was much greater than the non-dimensional term (PT) then the drift in the imaginary, out of axis, direction could be ignored. Where $T$ was defined in equation (2-4) and $\mathrm{P}$ was a non-dimensional roll rate factor, calculated by equation (2-9). Then, if the value inside the parenthesis was positive, the pitching moment coefficient was negative, then the projectile would tend to drift left. However, if the value inside the parenthesis was negative, the pitching moment coefficient was positive, then the projectile would tend to drift right (Wilson 2007).

$$
\begin{gathered}
\xi_{g}=\left(\frac{-P}{M+j P T}\right) g d V_{0}^{-2} \\
P=\frac{I_{x x}}{I_{y y}} \frac{p d}{V}
\end{gathered}
$$

When a projectile is not spinning, there is no gyroscopic stability force to create a restoring moment for the projectile's angle of attack. For a non-spinning projectile to be stable, it must be statically stable and dynamically stable. Being statically stabile means when the projectile was disrupted from its initial trajectory it will return to its steady state orientation. For the M781 to be statically stable, M of equation (2-10) must be less than zero. Also, from equation (2-10), it was found that $C_{m \alpha}$ must be negative for the projectile to be statically stable since all other variables were constant.

$$
M=\left(\frac{\rho A_{r e f} d^{3}}{2 I_{y y}}\right) C_{m_{\alpha}}
$$

A non-spinning projectile must also be dynamically stable so that perturbations in flight will not cause the angle of attack or beta angle to uncontrollably oscillate. The dynamic stability criterion for a non-spinning projectile must satisfy that equation (2-5) is greater than zero.

\subsubsection{Process of Designing a Stable Projectile}

A flow chart was created to determine the stability of a projectile, shown as Figure 2-2. When designing a stable projectile, a designer must first decide if the projectile will be launched from a rifled barrel, smooth bore tube or de-spun and leveled with control surfaces. Meaning the projectile was spinning or not-spinning. For a spinning projectile, gyroscopic stability must be checked and corrected if gyroscopically unstable. Static stability must be met if the projectile was launched from a smooth bore barrel or if the projectile was de-spun after launch. If not statically stable a non-spinning projectile must be aerodynamically redesigned so that the pitching moment coefficient was negative. Next, spinning and non-spinning projectiles need to have their dynamic stability analyzed and if unstable must have 
aerodynamic changes made to meet dynamic stability requirements. Projectiles must have their components placed so that the center of gravity of the projectile lies on the longitudinal-axis to avoid the projectile being laterally unstable. After the stability criteria for each type of projectile have been analyzed, the projectile may then be able to be constructed.

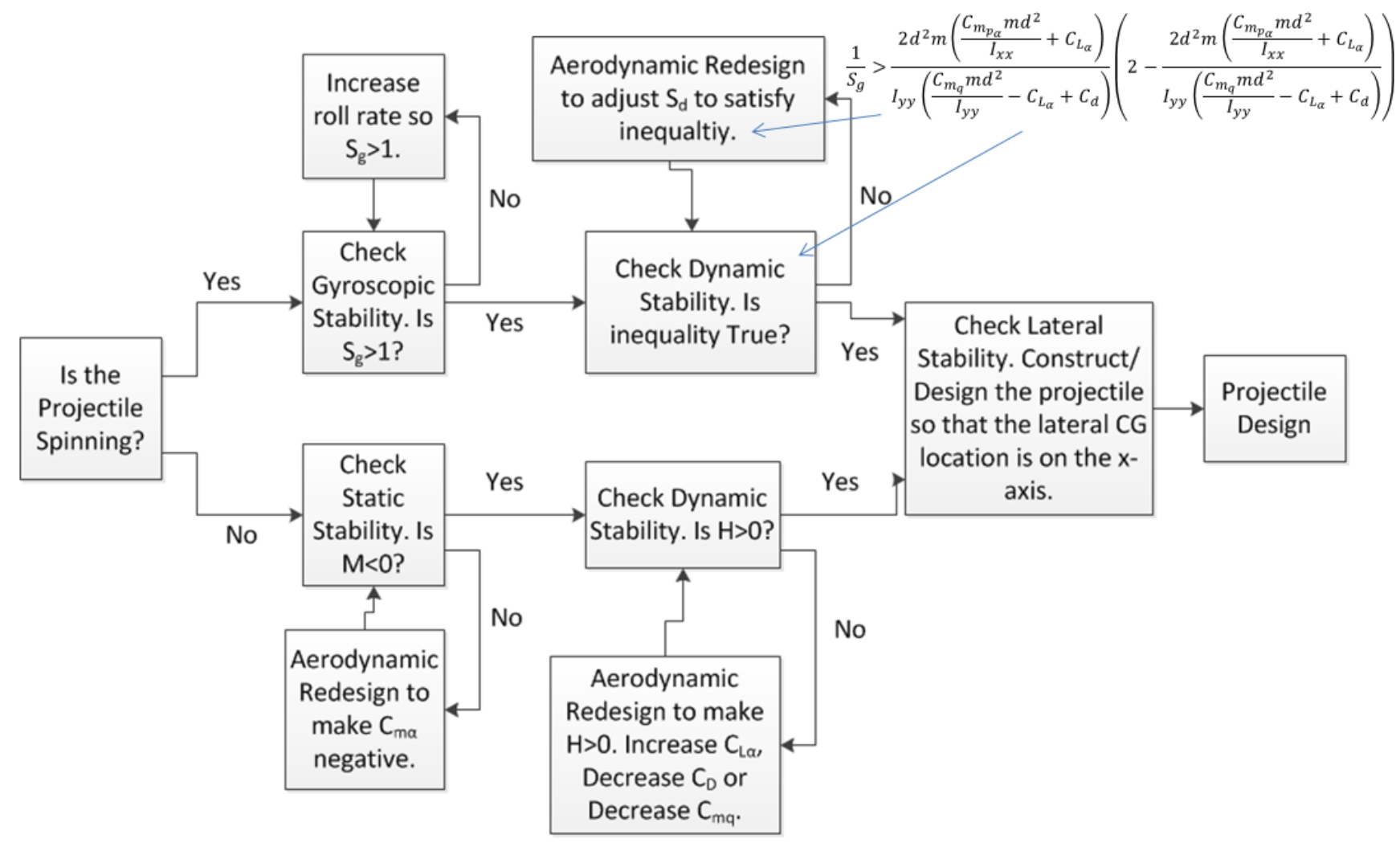

Figure 2-2: Flowchart to Check and Correct Stability of a Projectile 


\subsection{Modeling Flight using Equations of Motion}

Predicting the flight of a projectile required a Six Degree of Freedom (6DoF) simulation. A 6DoF simulation was used to numerically calculate the orientation and position for the projectile. Inside the simulator equations of motion were used to solve Newton's second law to balance inertial with external forces of the projectile. The earth fixed coordinate system used in flight applications was oriented from a specified ground location with the z-axis normal to the gravity vector, in the direction of gravity (Phillips 2010). The angles between the respective axes of Figure 2-3, (Matlab 2014) gave the roll, pitch and yaw angles ( $\varphi, \theta$ and $\psi$ respectively). In Figure 2-3 (X) and $(\mathrm{Y})$ represent the coordinate system axes. The (b) subscript means body referenced system and the (e) subscript means earth referenced system. The roll angle $(\varphi)$ was measured as the angle between $\mathrm{Y}_{\mathrm{b}}$ and $\mathrm{Y}_{\mathrm{e}}$ in the $\mathrm{Y}-\mathrm{Z}$ plane, the pitch angle $(\theta)$ was measured as the angle between $\mathrm{X}_{\mathrm{b}}$ and $\mathrm{X}_{\mathrm{e}}$ and the yaw angle $(\psi)$ was measured as the angle between $\mathrm{Y}_{\mathrm{b}}$ and $\mathrm{Y}_{\mathrm{e}}$ in the $\mathrm{X}-\mathrm{Y}$ plane.

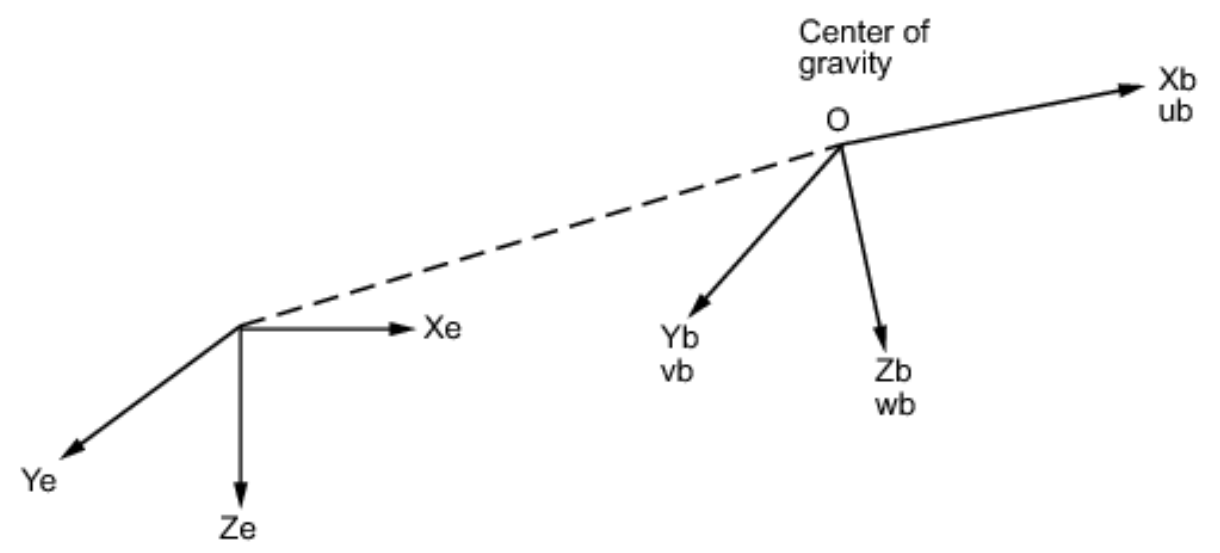

Flat Earth reference frame

Figure 2-3: Earth-Fixed and Body-Fixed Coordinate System

Time derivatives of linear and angular momentum were equal to the externally applied forces and moments, respectively. These balances were equated by integrating about the projectile. In equation (211), the left side of the equation was the linear momentum and the right side of the equation was the applied forces (Roskam 2003). Similarly, the left side of the equation (2-12) consisted of the angular momentum terms and the right side consisted of the applied moment terms.

$$
\begin{gathered}
\frac{d}{d t} \int_{V} \rho_{A} \frac{d \vec{r}^{\prime}}{d t} d V=\int_{V} \rho_{A} \vec{g} d V+\int_{S} \vec{F} d s \\
\frac{d}{d t} \int_{V} \vec{r}^{\prime} \times \rho_{A} \frac{d \vec{r}^{\prime}}{d t} d V=\int_{V} \vec{r}^{\prime} \times \rho_{A} \vec{g} d V+\int_{S} \vec{r}^{\prime} \times \vec{F} d s
\end{gathered}
$$


Where: $\rho_{A}$ was the projectile mass density,

$$
\begin{gathered}
\text { So, } m=\int_{V} \rho_{A} d V, \\
\text { And, } \frac{d m}{d t}=0
\end{gathered}
$$

Using the integral forms of Newton's second law in equations (2-11) and (2-12) and the body orientation of the projectile, the linear momentum and force terms along with the angular momentum and moment terms could be separated into their three directions and rotations. In equations (2-13) through (215), the earth referenced velocity vector and the orientation were known, and used to compile the linear force equations (Roskam 2003). The roll, pitch and yaw rate (p, q and r, respectively) were determined from equations (2-16) through (2-18), (Roskam 2003). Similar to the force equations, angular momentum and moments of inertia were able to be separated into equations (2-19) through (2-21), (Roskam 2003). Equations (2-13) through (2-15) and (2-19) through (2-21) were modified to remove forces and moments due to thrust, since no thrust force was applied for the projectile studied in this case.

$$
\begin{gathered}
M_{p}(\dot{u}-v r+w q)=-M_{p} g \sin \theta+F_{x} \\
M_{p}(\dot{v}+u r-w p)=M_{p} g \sin \varphi \cos \theta+F_{y} \\
M_{p}(\dot{w}-u q+v p)=M_{p} g \cos \varphi \sin \theta+F_{z} \\
p=\dot{\varphi}-\dot{\psi} \sin \theta \\
q=\dot{\theta} \cos \varphi+\dot{\varphi} \cos \theta \sin \varphi \\
r=\dot{\psi} \cos \theta \cos \varphi-\dot{\theta} \sin \varphi \\
I_{x x} \dot{p}-I_{x z} \dot{r}-I_{x z} p q+\left(I_{z z}-I_{y y}\right) r q=\ell \\
I_{y y} \dot{q}+\left(I_{x x}-I_{z z}\right) p r+I_{x z}\left(p^{2}-r^{2}\right)=m \\
I_{z z} \dot{r}-I_{x z} \dot{p}+\left(I_{y y}-I_{x x}\right) p q+I_{x z} q r=n
\end{gathered}
$$

Equations (2-13) through (2-15) and (2-19) through (2-20) then needed to be modified individually to determine the linear acceleration in the $\mathrm{x}, \mathrm{y}$ and $\mathrm{z}$ direction ( $\dot{\mathrm{u}}, \dot{\mathrm{v}}$ and $\dot{\mathrm{w}}$ respectfully) and the angular acceleration about the $\mathrm{x}, \mathrm{y}$ and $\mathrm{z}$ axis ( $\dot{\mathrm{p}}, \dot{\mathrm{q}}$ and $\dot{\mathrm{r}}$ respectfully). Those equations were algebraically manipulated so that the linear and angular accelerations could be calculated, using equations (2-22) through (2-27).

$$
\begin{gathered}
\dot{u}=\frac{F_{x}}{M_{p}}-g \sin \theta+r v-q w \\
\dot{v}=\frac{F_{y}}{M_{p}}+g \sin \varphi \cos \theta+p w-r u
\end{gathered}
$$




$$
\begin{gathered}
\dot{w}=\frac{F_{z}}{M_{p}}+g \cos \varphi \cos \theta+q u-p v \\
\dot{p}=\frac{\ell I_{z z}+n I_{x z}+p q\left(I_{x x} I_{x z}-I_{y y} I_{x z}+I_{z z} I_{x z}\right)+q r\left(I_{y y} I_{z z}-I_{x z}^{2}-I_{z z}^{2}\right)}{I_{x x} I_{z z}-I_{x z}^{2}} \\
\dot{q}=\frac{m+p r\left(I_{z z}-I_{x x}\right)+\left(r^{2}-p^{2}\right) I_{x z}}{I_{y y}} \\
\dot{r}=\frac{\ell I_{z x}+n I_{x x}+r q\left(I_{y y} I_{x z}-I_{x x} I_{x z}-I_{z z} I_{x z}\right)+q p\left(I_{x x}^{2}-I_{x x} I_{y y}+I_{x z}^{2}\right)}{I_{x x} I_{z z}-I_{x z}^{2}}
\end{gathered}
$$

In equations (2-22) through (2-27), initial conditions (mass, g, moments of inertia) were given. However the three directional forces and moments $\left(F_{x}, F_{y}, F_{z}, \ell, m\right.$ and $\left.n\right)$ were still needed to be calculated at each time step (Phillips 2010). These forces and moments were calculated by adding up all the external aerodynamic forces and moments by using an aerodynamic build-up of coefficients. Coefficients were used to solve for the total external forces and moments on the projectile. The external forces on the projectile (axial, side and normal forces) were calculated using the transformation in equations (2-28) through (2-30) and the orientation of the projectile to the freestream shown in Figure 2-4. Where the drag, lift and side lift coefficients $\left(C_{D}, C_{L}\right.$ and $\left.C_{S L}\right)$ were known from literature data or estimated through a build-up of aerodynamic coefficients.
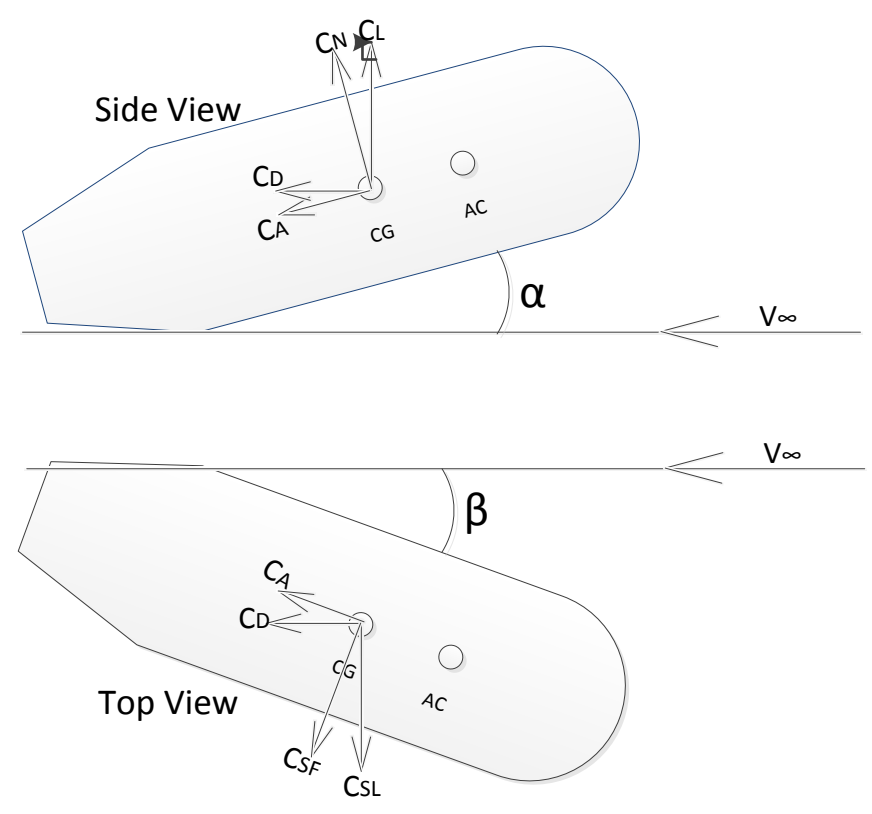

Figure 2-4: $C_{L}, C_{D}$ and $C_{S L}$ to $C_{N}, C_{A}$ and $C_{Y}$ 


$$
\begin{gathered}
C_{N}=C_{L} \cos (\alpha)+C_{D} \sin (\alpha) \\
C_{A}=-C_{L} \sin (\alpha)+C_{D} \cos (|\alpha|+|\beta|)-C_{S L} \sin (\beta) \\
C_{Y}=C_{S L} \cos (\beta)+C_{D} \sin (\beta)
\end{gathered}
$$

The total force and moment equations required to solve the equations of motion were compiled as equations (2-31) through (2-36). Where the rolling, pitching and moment coefficients $\left(C_{\ell}, C_{m}\right.$ and $\left.C_{n}\right)$ were also solved using literature data or an aerodynamic build-up. The pitching and yawing moments were multiplied by the reference length $\left(\ell_{\text {ref }}\right)$ which was assumed to be 0.1 meters.

$$
\begin{gathered}
F_{x}=.5 \rho A_{r e f} V^{2} C_{A} \\
F_{Y}=.5 \rho A_{r e f} V^{2} C_{Y} \\
F_{Z}=.5 \rho A_{r e f} V^{2} C_{N} \\
\ell=.5 \rho A_{r e f} \ell_{r e f} V^{2} C_{\ell} \\
m=.5 \rho A_{r e f} \ell_{r e f} V^{2} C_{m} \\
n=.5 \rho A_{r e f} \ell_{r e f} V^{2} C_{n}
\end{gathered}
$$

\subsection{Six Degree of Freedom Simulation of M781}

Using the coefficients and specifications found in literature for the M781, equations of motion were solved using Simulink's 6 DoF simulation. The simulation was used to estimate the trajectory of the M781 and examine control methods. A projectile flight simulator was used as the base of the model that will be used to determine the flight characteristics of this projectile (Wilhelm, et al. 2012). The highest level block of the model is shown in Figure 2-5. The 'Calc Forces' block was used to calculate the aerodynamic coefficients due to the projectile's orientation (States). These coefficients were then multiplied by the reference area, dynamic pressure and reference length to be converted to forces and moments. The equations of motion were then solved using the forces and moments described in Section 2.3. The $6 \mathrm{DoF}$ simulation then determined the earth-fixed velocity, earth-fixed position, earth-fixed orientation, body-fixed orientation, body-fixed velocity, body-fixed angular rate, body-fixed angular acceleration and body-fixed accelerations for the M781. 


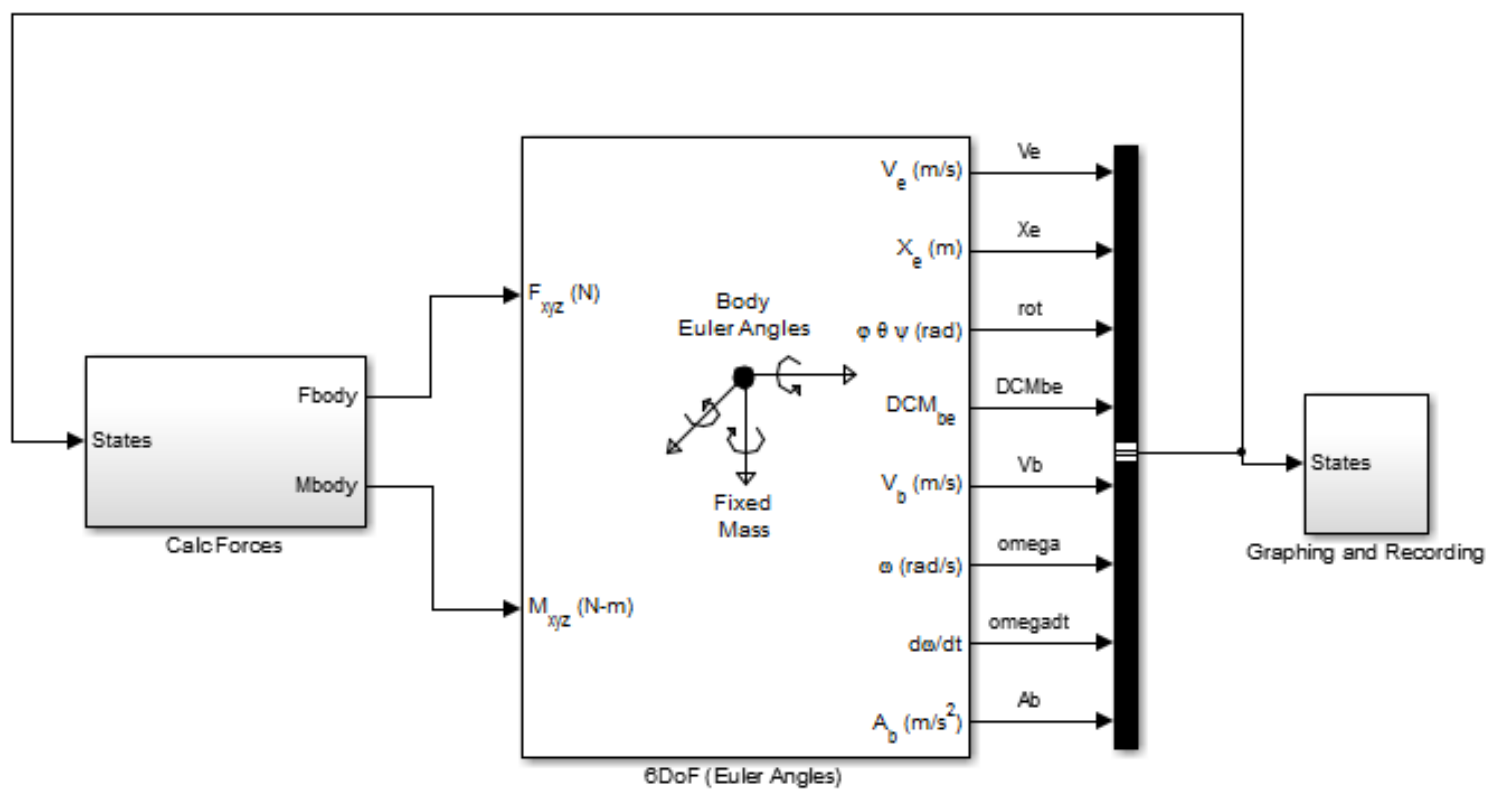

Figure 2-5: Simulation Model

\subsubsection{M781 Model Verification}

The 6DoF model that was constructed for this work was verified for accuracy by matching its simulation results with two different cases for the M781. The cases that the model was compared against were literature data for maximum range and literature simulation results for the M781. Using the coefficients and initial conditions compiled in Table 2-1 the M781 was simulated and compared with results from literature.

In multiple literature sources the maximum range for the M781 $40 \mathrm{~mm}$ projectile was found to be 400 meters when landing at the same altitude that it was launched (Cooke 2010) (MLM International 2005) (ALS Technologies 2009). However, in these literature sources the launch elevation angle was not given for the maximum range condition. So the launch angle that yielded the farthest range was determined by simulating all launch angles with the specifications found in Table 2-1. From Figure 2-6 the launch angle for the maximum range condition was found to be 42 degrees. This launch angle was then simulated and its trajectory was shown in Figure 2-7. Maximum range for the simulated M781 was found to be 408.1 meters. Meaning the range found using this 6 DoF simulation was $2 \%$ different than maximum range of 400 meters found in literature for the M781. 


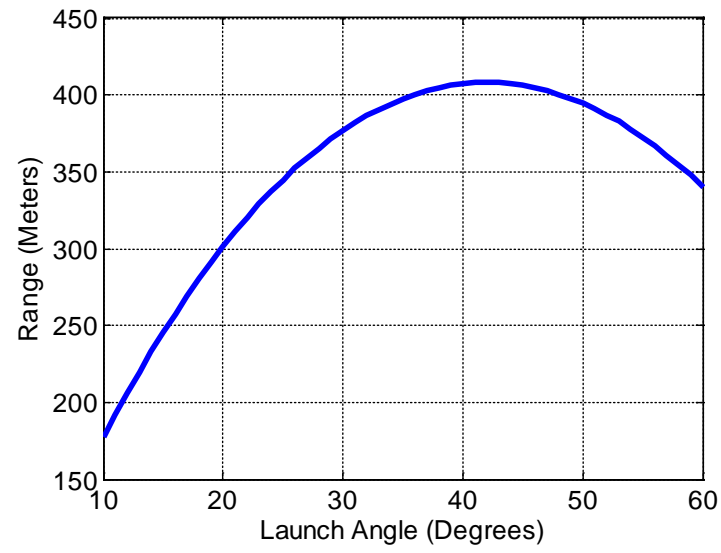

Figure 2-6: Simulation to Determine Max Range Launch Angle for M781

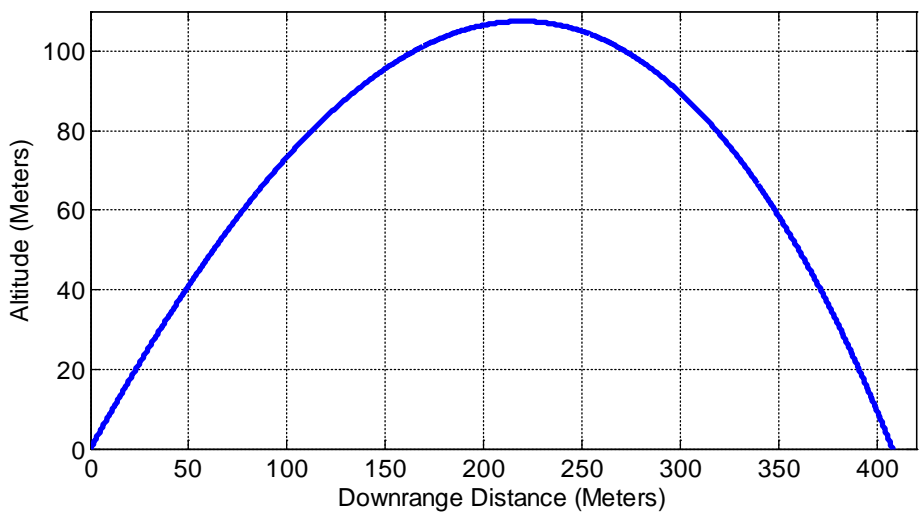

Figure 2-7: M781 Trajectory for 42 Deg. Launch Angle

The trajectory for the M781 from the 6DoF model was also compared with simulation results in (Gkritzapis, et al. 2008). Figure 2-8 shows the simulation results from their simulation for launch angle of 14.7, 30 and 40 degrees. The model created in this work was used to simulate the trajectories for the same launch angles, shown as Figure 2-9. Results from the two simulations are compared for the two sets of trajectories in Table 2-2. In Table 2-2 the results of each set of simulations were compiled and it was shown that each set of simulation results varied by a maximum of $0.21 \%$ in final distance and apex altitude. 


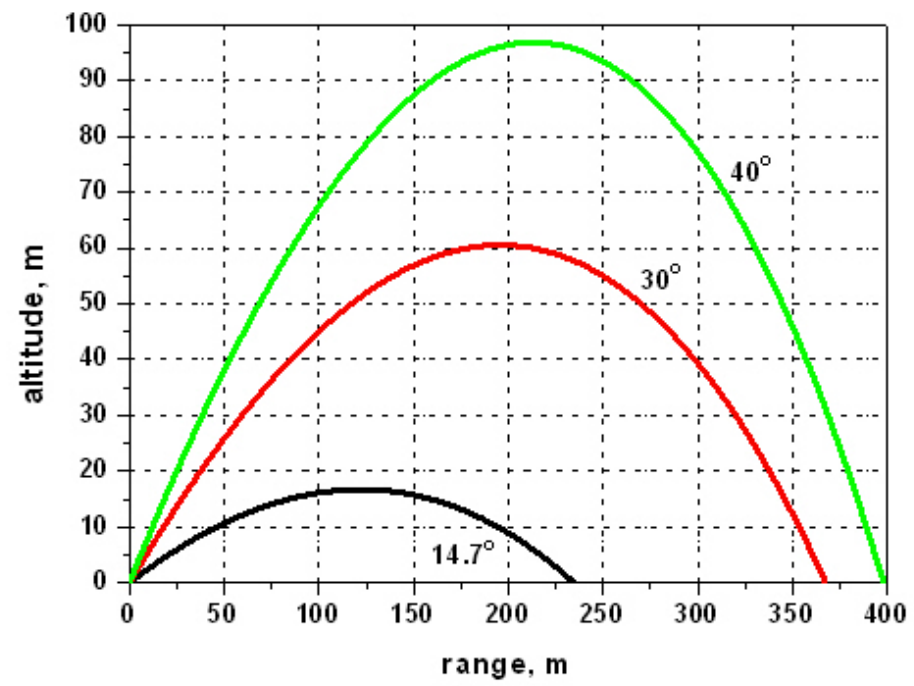

Figure 2-8: Flight Path trajectories for $40 \mathrm{~mm}$ projectile from (Gkritzapis, et al. 2008)

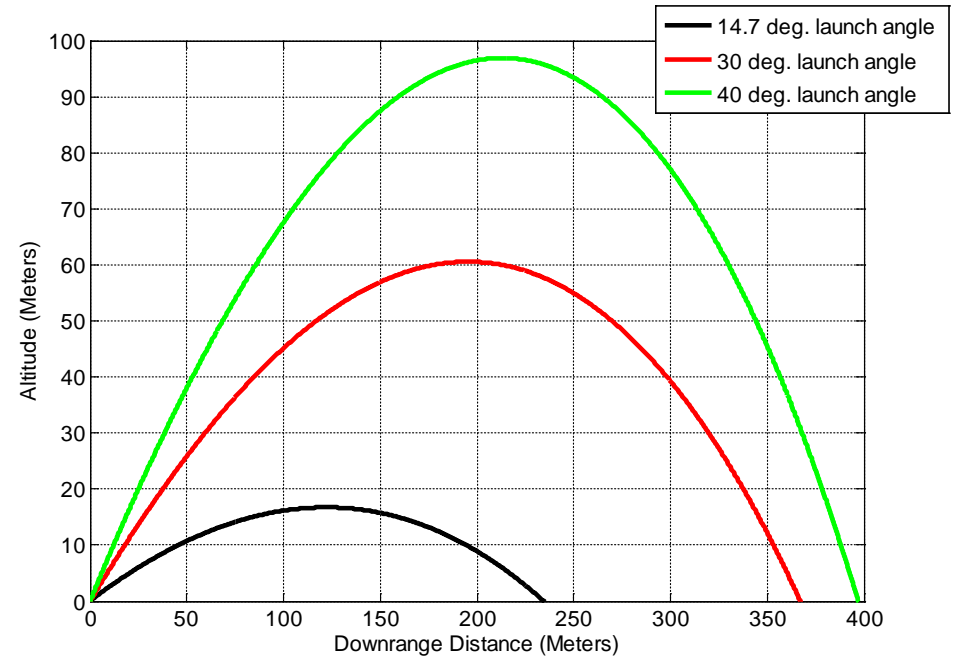

Figure 2-9: Flight Path Trajectories from Created Model

Table 2-2: Maximum Range and Altitude from Simulation and Literature Results

\begin{tabular}{|r|c|c|c|c|c|c|}
\hline & \multicolumn{2}{|c|}{$\begin{array}{c}\text { Source (Gkritzapis, et al. } \\
\text { 2008) }\end{array}$} & \multicolumn{2}{c|}{ Model created in this Study } & \multicolumn{2}{c|}{ Percent Difference } \\
\hline & Distance (m) & Altitude(m) & Distance(m) & Altitude(m) & Distance(m) & Altitude(m) \\
\hline 14.7 degrees & 235 & 16.7 & 234.86 & 16.74 & $0.05 \%$ & $0.2 \%$ \\
\hline 30 degrees & 367 & 60.5 & 367.13 & 60.57 & $0.04 \%$ & $0.1 \%$ \\
\hline 40 degrees & 398 & 97.0 & 397.17 & 96.90 & $0.21 \%$ & $0.1 \%$ \\
\hline
\end{tabular}


Results from these simulations show that the model accurately predicts the flight of the M781 compared to other literature sources. Also, the coefficients found in literature were accurate for the projectile and continued to be used as the coefficients of body during the HP build-up. A control system of surfaces and a controller could now be added to the model and as long as the control model was meticulously designed then the model should remain accurate.

\subsection{Investigate other HP's for Control System Methods}

Three different hybrid projectile designs were investigated to evaluate modifications that were made to adapt a standard projectile to a HP. The upgrades investigated were added to the projectiles to increase range and add guidance capabilities. Altercations include control surfaces and controllers added to maneuver the HPs. All three of the literature designs used a symmetric spin-stabilized projectile, similar to the M781, which remained spinning during flight.

The first projectile investigated was a $40 \mathrm{~mm}$ guided round designed by Stellar Exploration, Figure 2-10 (Exploration 2011). Range extension was achieved for this HP by adding a solid rocket motor inside which added thrust during flight. The Stellar Exploration $40 \mathrm{~mm}$ has an advertised maximum range of 800 meters with a circular error probability of one meter. The propulsion system and wings allowed this projectile to achieve a 100\% range extension over a standard $40 \mathrm{~mm}$ projectile. Control surfaces on the Stellar Exploration HP were wings that deploy from the side of the HP used to make a force normal to its roll angle. These wings are set at a specified incidence angle and were only effective for the portion of the flight in which they were deployed. Since this projectile remained spinning, it required a flight control system to stow and deploy the wings at the correct angle in its rotation to make a desired maneuver.

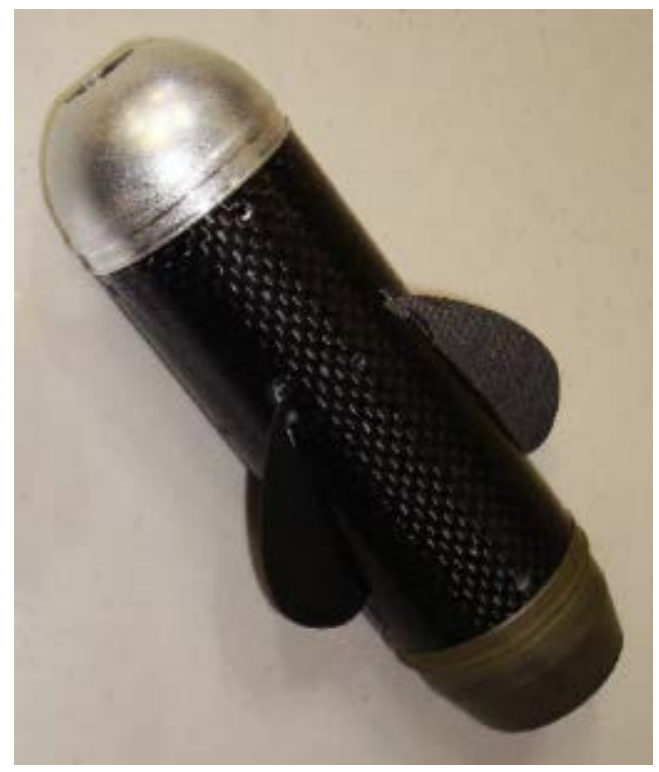

Figure 2-10: Stellar Exploration 40 mm Guided Munition (Exploration 2011) 
DARPA also modified a standard $40 \mathrm{~mm}$ munition to a HP called the scorpion, in Figure 2-11 (Lovas, et al. 2009). The Scorpion used synthetic jet actuator as its control surface for maneuvering. The synthetic jet actuator was used to keep the projectile on course (McMichael, et al. 2004). This projectile had guidance capability only to be held on its ballistic trajectory because flow control was only used to correct the Magnus drift of the projectile. This control type was used to reduce drift to its desired impact location, increasing accuracy.
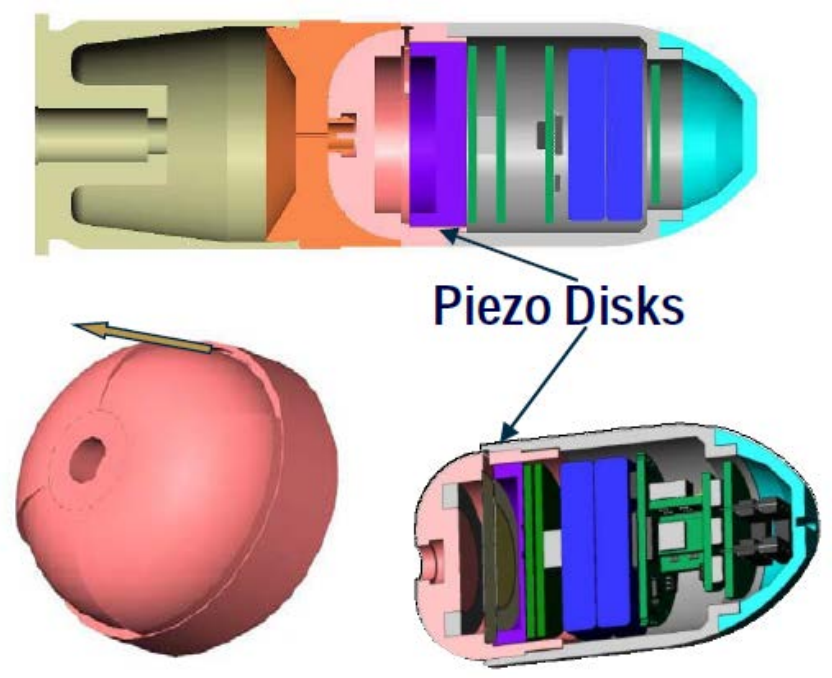

Figure 2-11: DARPA Scorpion (Lovas, et al. 2009)

The final design examined was a $155 \mathrm{~mm}$ round where the baseline projectile, a Howletzer, was modified with a rotating puck used as a control surface, Figure 2-12 (Fresconi, et al. 2011). This puck was able to add 700 meters of control authority for the 22,000 meter flight of their specific 155 mm projectile (Fresconi, et al. 2011). If this control authority ratio was applied to a $40 \mathrm{~mm}$ projectile range of 400 meters, there would be 12 meters of control authority. Since there was only one control surface, heading and longitudinal control were coupled and control authority was measured in range extension and side movement combined. 


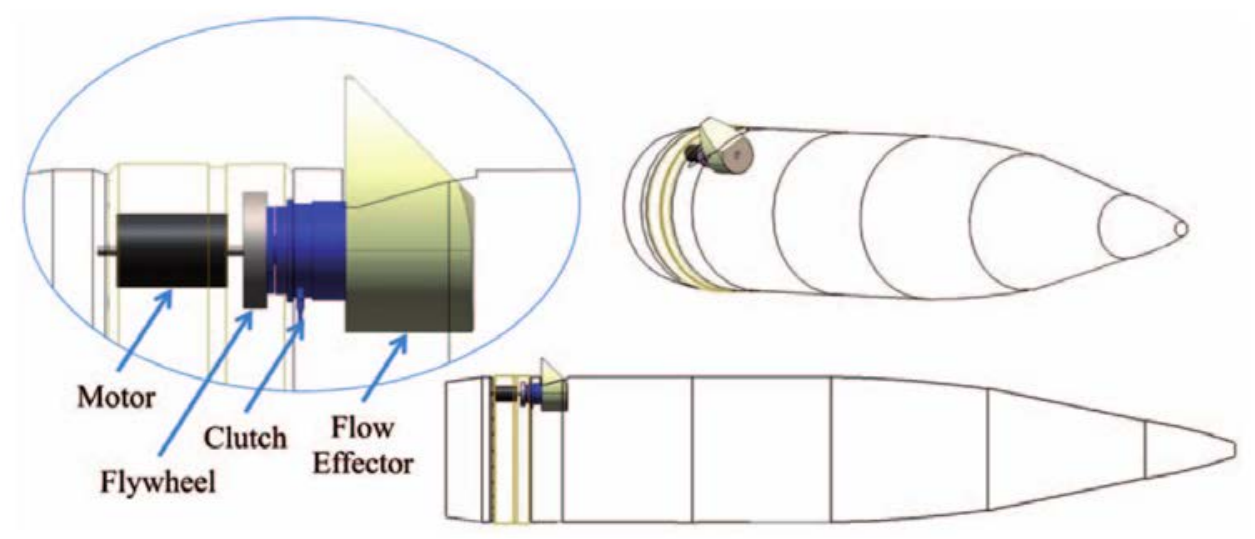

Figure 2-12: 155mm Puck Control Projectile (Fresconi, et al. 2011)

The previously designed guidable projectiles found in literature remained spinning while being controlled. First, spinning projectiles have a Magnus force that must be counteracted as well as a yaw of repose component which pulls them off their original line of fire (Fresconi, et al. 2011) (Wilson 2007). Second, a spinning projectile would require that the control surfaces only be used at a specific roll angle, reducing total control authority. Finally, since the body remained spinning the control surfaces could not hold the projectile at a constant positive angle of attack.

\subsection{Control Methods for Aircraft Guidance}

Aircraft autopilot control methods were investigated to create basic knowledge of concepts that could be modified for a HP. Traditional manned aircraft and UAV's have autopilot systems to achieve specific guidance goals of the aircraft. Autopilots are used to do tasks from altitude holding, velocity holding, heading tracking and many others. The methods that were used for the HP being adapted in thesis were heading tracking and altitude holding. However, these methods would need to be modified to be useful on a projectile with no main wing, no propulsion and a cruciform tail section.

\subsubsection{Heading Control}

A roll controller for a manned or unmanned aircraft was used to deflect ailerons (on main wing) and the rudder (on vertical tail) to create a rolling torque to keep the aircraft wings-level in flight (Christiansen 2004). When an aircraft is at a roll angle, other than zero, the wing creates a lifting force in the horizontal direction, causing horizontal movement. Meaning that roll and yaw control for a winged aircraft must be coupled, known as lateral-directional control (Roskam 2003). 
In (Christiansen 2004), lateral-direction control was shown as inner and outer loop that was used to maneuver the aircraft to a desired heading. The outer loop of the lateral-directional controller, shown as Figure 2-13, was used to create an output of desired roll angle to drive the heading error to zero. In this case, a controller used to drive the heading error to zero was a proportional, integral and derivative (PID) controller. This roll angle was used as the input to Figure 2-14, in which a proportional and integral (PI) controller drove the roll angle error to zero by creating aileron deflections. The ailerons on a manned aircraft are also used to damp the roll rate created during a roll maneuver as the upper summation path of Figure 2-14 (Christiansen 2004). Roll damping is necessary so harsh roll maneuvers were not felt by passengers. In Figure 2-15, rudder deflection is used to cause the aircraft to yaw at particular rate.

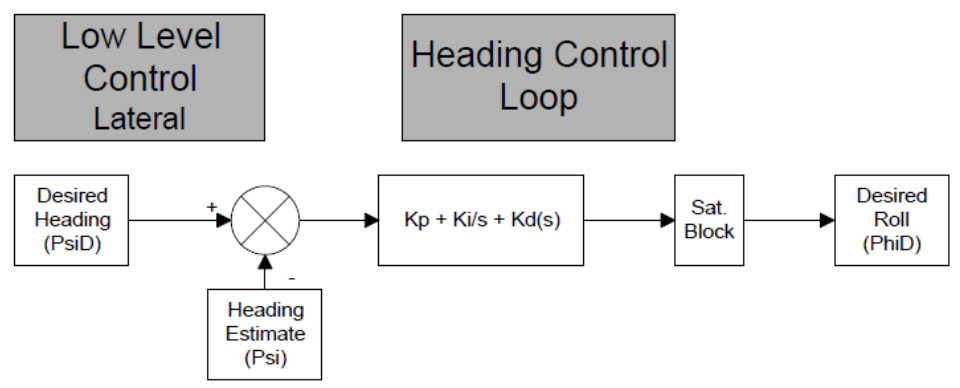

Figure 2-13: Outer Lateral Heading Angle Controller (Christiansen 2004)

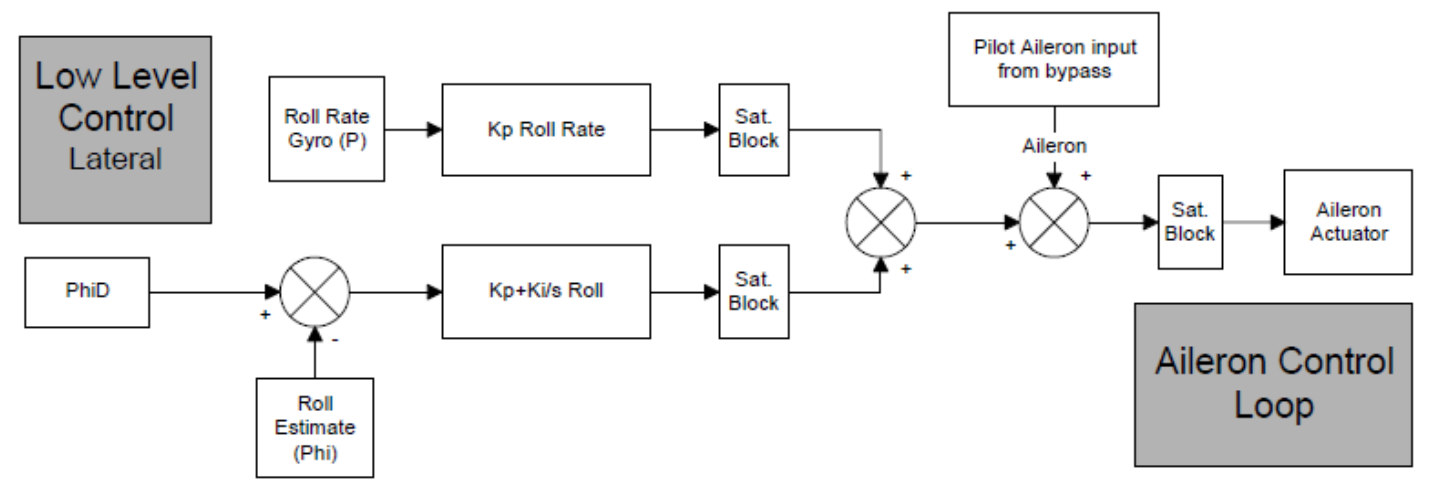

Figure 2-14: Inner Lateral Roll and Roll Rate Control (Christiansen 2004)

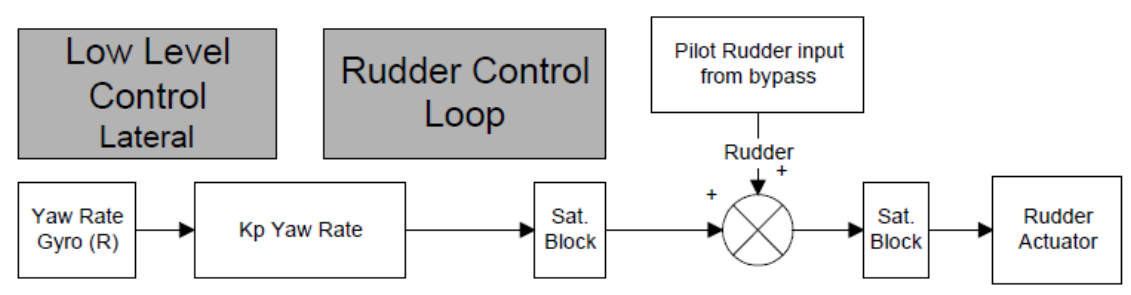

Figure 2-15: Inner Lateral Yaw Rate Controller (Christiansen 2004) 
The basic control system found, led to an initial design envisioned for the heading controller used in this work. The HP in this study only has vertical tails for heading maneuvering which act like the rudder on the aircraft in the example.

\subsubsection{Longitudinal Control}

Longitudinal control of a manned or unmanned aircraft normally contains two separate controllers. The first, is an altitude controller which uses horizontal tail deflections to change the pitch angle of the aircraft until it reaches a desired altitude. Second, an airspeed controller that adjusted the throttle position to increase thrust until the aircraft reaches a desired velocity (Ramezani 2012). The outer loop of the altitude controller uses a PID controller to drive the altitude error to zero by determining the desired pitch, Figure 2-16. The inner loop of the altitude controller determines the required elevator deflection to drive the pitch angle to the desired value, determined in the outer loop. This controller compensates for roll and limits the pitch rate, Figure 2-17 (Christiansen 2004). The airspeed controller, Figure 2-17, uses a PID controller the drive the airspeed error to zero by changing throttle position to reduce or increase thrust.

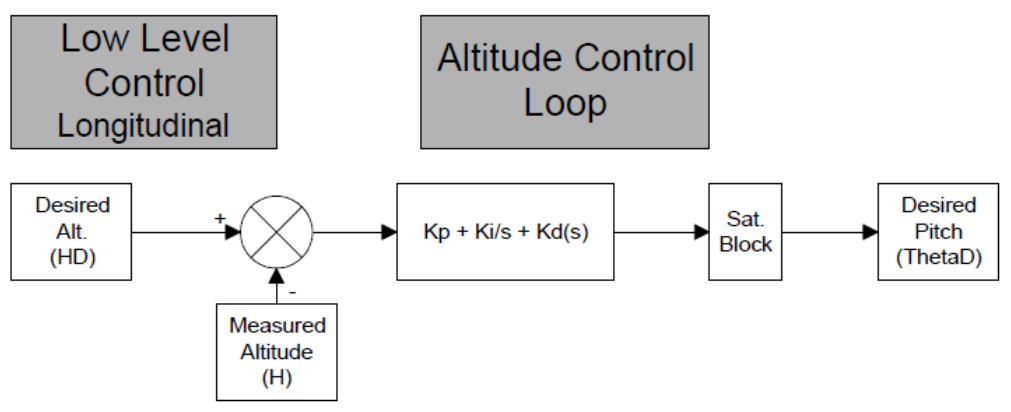

Figure 2-16: Outer Longitudinal Altitude Controller (Christiansen 2004)

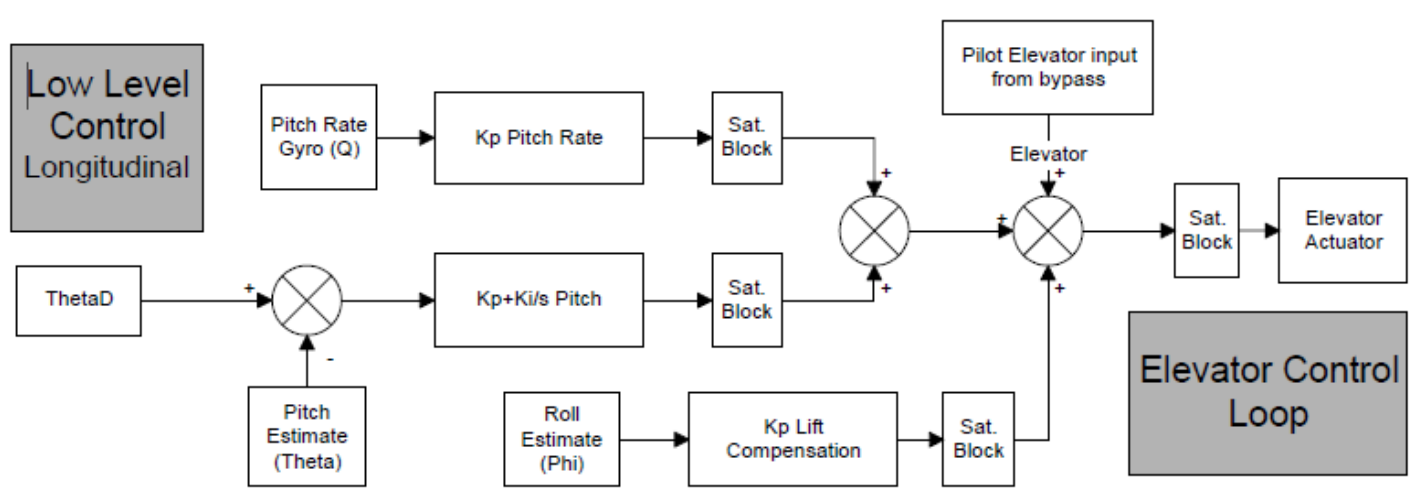

Figure 2-17: Inner Longitudinal Airspeed Controller (Christiansen 2004) 


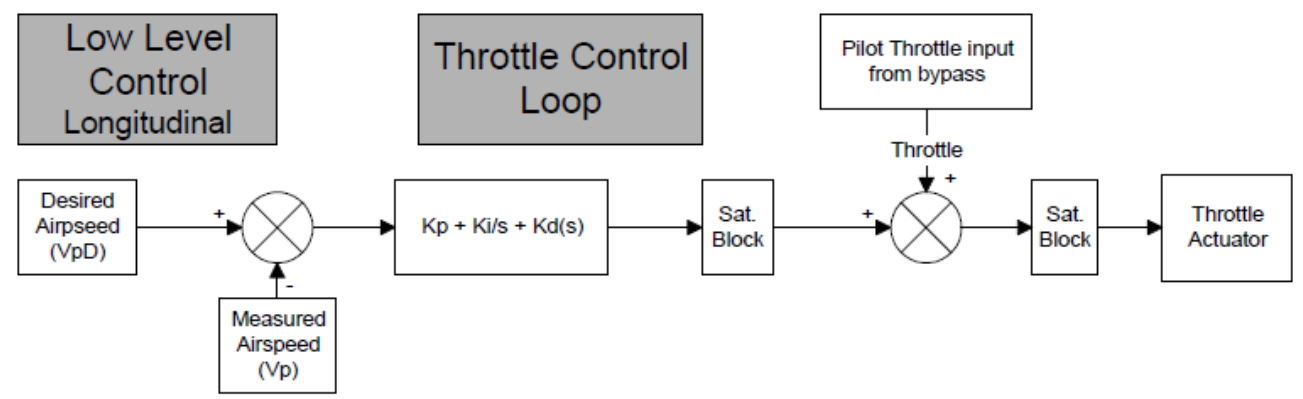

Figure 2-18: Inner Longitudinal Airspeed Controller (Christiansen 2004)

The longitudinal control method investigated in this thesis could not use an airspeed controller since the HP did not have direct means of increasing its velocity after it was launched. There was no propulsion system on the HP in this study so an altitude hold maneuver was not possible since there was no way of holding a particular altitude using thrust. If an altitude were to be held its velocity would continually drop due to drag and the tradeoff of kinetic to potential energy. However, the idea of using horizontal tail (elevator) deflection for pitch controller was utilized to increase the angle of attack so the body could produce lift extending range without the PID controller. A different loop configuration needed to be created for the HP to reach a desired altitude at a desired downrange distance while following a ballistic trajectory.

\subsection{Controllers for Aircraft Application}

Controllers have been used in different applications for many years. They can be used for HVAC systems, electrical amplification and aerospace applications, etc. In aerospace applications, there were many different types of controllers that could be used to achieve the designers desired output. From the large variety of controllers available, a few were investigated for use in the autopilots of this Hybrid Projectile. 
In (Haiyang Chao 2007) control techniques were investigated, including; PID, Neural Network, Fuzzy Logic, Sliding Mode and $\mathrm{H}_{\infty}$ control in autopilots (Haiyang Chao 2007). PID was chosen as the desired control type because it was simple to use, had three tunable gain values and could be implemented in small UAV applications (Haiyang Chao 2007). The multiple gain values include; proportional, integral and derivative gain that can be tuned individually to get a desired output. In this controller, the proportional gain was used to decrease rise time, decrease steady state error and slightly change settling time but increase overshoot (Matlab 2014). The integral gain was used to eliminate steady state error but resulted in an increase of overshoot and settling time (Matlab 2014). The derivative gain was used decrease overshoot, slightly change rise time and decrease settling time but did not change steady state error (Matlab 2014). Characteristics of the PID controller were found in Matlab's introduction to PID controller design. The other controller types could be used but in this case, for the HP being modified, a PID controller was able to achieve all the goals that were required.

\subsection{Literature Conclusions}

Through the use of background literature research, a plan was determined to achieve the goal of adapting a M781 to a HP with the use of simulation. The baseline coefficients and geometry were collected from literature for the M781 projectile which was used as the body of the HP. Stability equations were then found that could be used to analyze the stability of the projectile by knowing its geometry and aerodynamic. Equations of motion were then found to be used to predict the flight of the HP. Forces and moments due to aerodynamics and gravity were required to solve the equations of motion that could be estimated due to an aerodynamic coefficient build-up. Simulink's aerospace toolbox was used to build a model for the HP to apply controllers and predict performance of guidance capabilities. Current HPs were then investigated for information on types of control surfaces and methods to achieve guidance. Control methods were researched for aircraft since the HP acted like a UAV while in the guidance portion of its flight. Types of controllers were then found so advantages and disadvantages could be compared. It was chosen that PID control be used since it had multiple gain values that could be used to fine tune guidance performance. This key information was used to create an idea that would be implemented throughout this thesis. 


\section{Chapter 3 Control and Stability for a $40 \mathrm{~mm} \mathrm{HP}$}

\subsection{Stability Analysis for the M781}

When adapting a M781 to a HP it was necessary to determine if the M781 was stable, so that the projectile does not tumble and become uncontrollable. First, stability was analytically estimated for the M781 while spinning then estimated if the M781 was not spinning. After analyzing equation (2-1) for a standard spinning M781 $40 \mathrm{~mm}$ round, the gyroscopic stability factor $\left(\mathrm{S}_{\mathrm{g}}\right)$ was calculated to be 3.51 using the values compiled in Table 2-1, meaning this projectile was gyroscopically stable. Solving the inequality for the projectile with the values in Table 2-1, it was estimated that the M781 was dynamically stable since equation (2-7) was true. Calculations for gyroscopic and dynamic stability are provided in Appendix A.

However, in this study the HP being adapted was desired to be non-spinning so that the projectile could be held at an increased angle of attack and the body could be used as a lifting surface. In Appendix A, the static stability was also analyzed for a standard M781 projectile to determine if it was stable when not-spinning. It was calculated that the M781 was statically unstable because its CG was aft of the AC, therefore some type of control surface must be added to make it statically stable.

\subsection{Adaptations Required for Stability of M781 to a $40 \mathrm{~mm} \mathrm{HP}$}

The M781 required modification by adding tails to the projectile to transform into a HP so that it would be statically stable. Tail surfaces were chosen due to their tendency to increase static stability, pitching the nose down as angle of attack increases. These tail surfaces could also be utilized as control surfaces, if actuated, to maneuver the HP. The contribution of tails to the projectile was estimated due to a build-up of aerodynamic coefficients so that the HP's forces and moments could be calculated. The forces and moments were calculated using equations (2-31) through (2-36) so that the equations of motion could be solved for the HP. This meant that the total force and moment coefficients were required for the projectile body with the contribution of the tail surfaces. Using a coefficient build-up also allowed for multiple tail geometries to be considered. By changing the span, chord and location of the tails, the values of the aerodynamic coefficients changed. These coefficients were calculated for multiple tail geometries to determine which tail sizing ensured that stability was achieved according to the stability requirements in Section 2.2. 


\subsubsection{Aerodynamic Coefficients Contribution of Tail Surfaces}

Adding tails to a projectile changes the aerodynamic forces and moments, and in turn changes its flight. These changes were accounted for by adding the coefficient contribution of the tails. The added aerodynamic coefficients from the tail were then used to estimate the behavior of the actual projectile. The vertical and horizontal tails deflect positive and negative with respect to the freestream velocity as shown in Figure 3-1, as $\delta_{\mathrm{VT}}$ and $\delta_{\mathrm{HT}}$. In Figure 3-1 the tail deflections are shown in their positive direction with respect to the freestream. The coefficient build-up begins from the coefficients for the M781 with the added effect of the tails. The M781 coefficients from Table 2-1 were denoted in the coefficient build-up with a (Body) subscript.

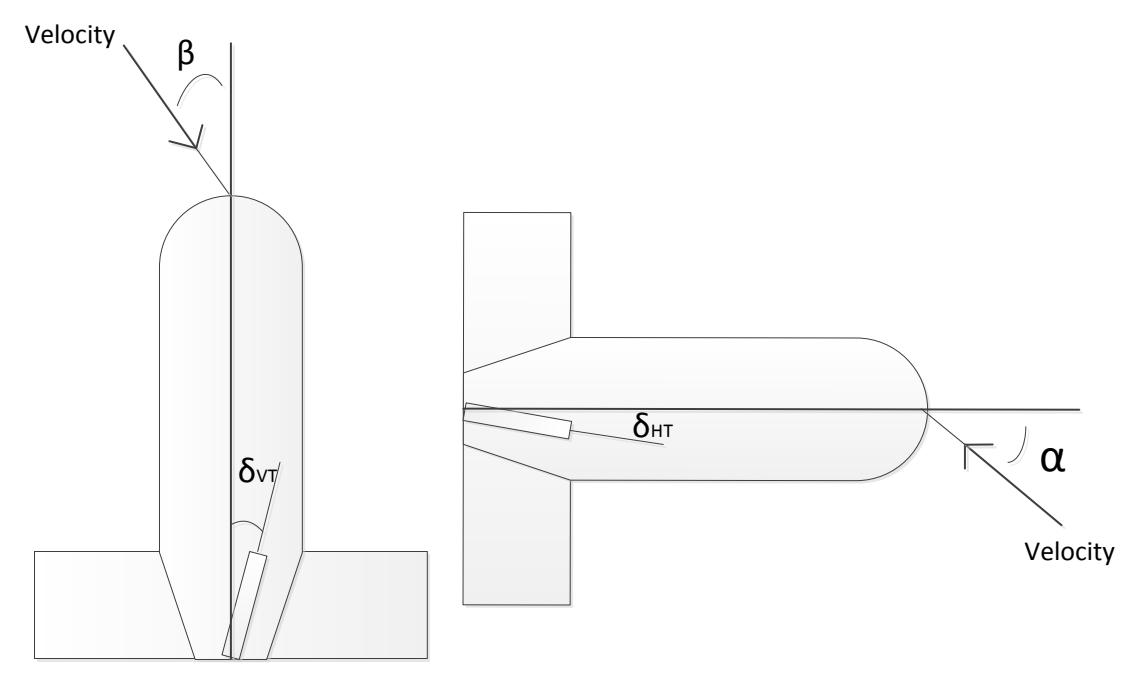

Figure 3-1: Tail Deflections With Respect to Freestream Velocity

\subsubsection{Drag}

The total drag of the projectile was determined by adding the drag contribution from the tails to the contribution from the projectile's body. The total drag force was determined by adding the drag coefficient of the body from Table 2-1 to the additional coefficient values determined for the designed tail surfaces. It was assumed that the tail surfaces used were flat plates. The drag of the tail was comprised of its zero lift drag $\left(C_{D_{0_{\text {Tail }}}}\right)$ as well as induced drag $\left(C_{D_{i_{\text {Tail }}}}\right)$. The zero lift drag was calculated by using the skin friction drag of a turbulent flat plate, equation (3-1), as given by Prandtl and found in (White 2006). The turbulent value was used since the Reynolds number of the tails were in the turbulent range. The lift induced drag by the tails caused an increased drag coefficient due to the magnitude of deflection from the freestream velocity. The tail deflection magnitudes were shown in Figure 3-1 and were $\alpha-\delta_{H T}$ for the horizontal tails and $\beta+\delta_{V T}$ for the vertical tails. In equation (3-2), the tail deflection made by the roll controller $(\delta)$ must be multiplied by two since $C_{L_{\delta_{H T}}}$ was calculated for two tails and four tails were 
defected for de-spin and roll-leveling control. The drag coefficient build-up of equation (3-3) included a value " 2 " before the number of tails $(\mathrm{N})$ which stands for the two sides of a flat plate (White 2006).

$$
\begin{gathered}
C_{D_{0_{\text {Tail }}}}=\frac{0.058}{\operatorname{Re}_{L}{ }^{1 / 5}} \\
C_{D_{i_{\text {Tail }}}}=\frac{\left[C_{L_{\delta_{H T}}}\left(\left|\alpha+\delta_{H T}\right|+\left|\beta-\delta_{V T}\right|+2|\delta|\right)\right]^{2}}{\pi e A R} \\
C_{D_{\text {Total }}}=C_{D_{\text {Body }}}+C_{D_{0_{\text {Tail }}}}\left(\frac{2 N A_{\text {tail }}}{A_{\text {ref }}}\right)+C_{D_{i_{\text {Tail }}}} \frac{N A_{\text {Tail }}}{A_{\text {ref }}}
\end{gathered}
$$

\subsubsection{Lift}

The lift additions due to the tail surfaces were determined next. The lift coefficients at zero degrees angle of attack and with change in tail deflection were examined. The lift of the tail with zero degrees of tail deflection was $\left(C_{L_{o_{\text {Tail }}}}=0.0\right)$ since the tail surfaces were assumed as flat plates, with no camber. The lift coefficient of the tail with tail deflection was given by equation (3-4), which takes into account the aspect ratio $\left(\mathrm{AR}_{\text {Tail }}\right)$ of the tail surfaces, as given by Prandtl lifting-line theory. There was also a contribution of lift coefficient due to rate of angle of attack change from the horizontal tails in equation (3-5), (Roskam 1971). In equation (3-5) the tail efficiency ( $\eta$ ) used was assumed to $90 \%$ due to the tails being in the wake of the body while it was being used as a lifting surface. The build-up of the total lift coefficient, equation (3-6), was compiled using a similar set-up as in (Phillips 2010). In equation (3-6) the tail deflection magnitude lift coefficient was based on $\alpha-\delta_{H T}$, as seen in Figure 3-1.

$$
\begin{aligned}
& C_{L_{\delta H T}}=\frac{2 \pi}{\left(1+\left(\frac{2}{A R_{\text {Tail }}}\right)\right)} \\
& C_{\dot{\alpha}_{H T}}=2 \eta C_{L_{\delta H T}} \\
& C_{L_{\text {Total }}}=C_{L_{\alpha_{B o d y}}} \alpha+C_{L_{\delta H T}}\left(\alpha-\delta_{H T}\right) \frac{N A_{\text {Tail }}}{2 A_{\text {ref }}}+C_{L_{\dot{\alpha}_{H T}}}\left(\frac{\dot{\alpha} d}{2 V}\right) \frac{N A_{\text {Tail }}}{2 A_{\text {ref }}}
\end{aligned}
$$




\subsubsection{Pitching Moment}

The pitching moment effects introduced by adding tails were created by the projectile spinning and the tail surfaces having an effective angle of attack. The pitching moment due to the tails being deflected $\left(C_{m_{\delta H T}}\right)$, equation (3-7), was determined from the flat plate lift coefficient. The pitching moment due to the roll rate of the projectile $\left(C_{m_{p_{T a i l}}}\right)$, equation (3-8), was added because of the recovery moment created to drive the projectile to zero degrees angle of attack. In the build-up of the pitching moment coefficient, equation (3-11), $C_{m_{p_{\text {Tail }}}}$ was multiplied by the sign of the angle of attack because of its tendency to pitch down with a positive angle of attack and pitch up with a negative angle of attack. Equation (3-9) was used to estimate the pitch damping moment coefficient contribution from the horizontal tail surfaces $\left(C_{m_{q_{H T}}}\right)$. The horizontal tail contribution to pitching moment due to the rate of change in angle of attack $\left(C_{m_{\dot{\alpha}_{H T}}}\right)$ was shown as equation (3-10). The total pitching moment $\left(C_{m_{\text {Total }}}\right)$ coefficient was built up as equation (3-11).

$$
\begin{aligned}
& C_{m_{\delta H T}}=\frac{-\eta 2 \pi}{\left(1+\left(\frac{2}{A R_{\text {Tail }}}\right)\right)} \\
& C_{m_{p_{\text {Tail }}}}=\frac{-\left(.6 \eta N A_{\text {tail }} l_{t}^{3}\right)}{A_{\text {ref }} d}\left(\frac{p^{2}}{V^{2}}\right) \\
& C_{m_{q_{H T}}}=-2.2 \eta C_{L_{\delta H T}}\left(\frac{\pi}{180}\right) \\
& C_{m_{\dot{\alpha}_{H T}}}=-2 \eta C_{L_{\delta H T}}\left(\frac{\pi}{180}\right) \\
& C_{m_{\text {Total }}}=C_{m_{\alpha \text { Body }}}(\alpha)+C_{m_{\delta H T}}\left(\frac{\left(\alpha-\delta_{H T}\right) \pi}{180}\right)\left(\frac{l_{\text {tail }}}{l_{\text {ref }}}\right)\left(\frac{N A_{\text {Tails }}}{2 A_{\text {ref }}}\right)+C_{m p_{\text {tail }}}(\operatorname{sign}(\alpha)) \\
& +\left(C_{m_{q_{H T}}}+C_{m_{q \text { Body }}}\right)\left(\frac{\left(\frac{180}{\pi}\right) q d}{2 V}\right) \frac{N A_{\text {Tail }}}{2 A_{\text {ref }}}\left(\frac{l_{\text {tail }}}{l_{\text {ref }}}\right)^{2} \\
& +C_{m_{\dot{\alpha}_{H T}}}\left(\frac{\dot{\alpha} d}{2 V}\right) \frac{N A_{\text {Tail }}}{2 A_{\text {ref }}}\left(\frac{l_{\text {tail }}}{l_{\text {ref }}}\right)^{2}
\end{aligned}
$$




\subsubsection{Side Force}

The side lift force refers to the force in the y-direction, which is similar to lift in the z-direction. The side lift force coefficient used the same build up components as lift because the munition was axisymmetric. In equation (3-12) the side lift force coefficient from vertical tail deflection $\left(C_{S L_{\delta V T}}\right)$ was the same as equation (3-4) because the horizontal and vertical tails being used have the same geometry. The deflection magnitude multiplied by the lift coefficient due to tail deflection is $\beta+\delta_{V T}$, which was defined in Figure 3-1. Equation (3-13) was the side lift force coefficient contribution from the vertical tails due to the rate of change in beta angle $\left(C_{S L_{\dot{\beta}_{V T}}}\right)$. The total side lift force coefficient $\left(C_{S L_{T o t a l}}\right)$ was combined as equation (3-14). The only difference to equation (3-14) from equation (3-6) was that the coefficients were multiplied by $\beta$ instead of $\alpha$.

$$
\begin{aligned}
& C_{S L_{\delta V T}}=\frac{2 \pi}{\left(1+\left(\frac{2}{\text { AR }}\right)\right)} \\
& C_{S L_{\dot{\beta}_{V T}}}=2 \eta C_{L_{\delta H T}}
\end{aligned}
$$

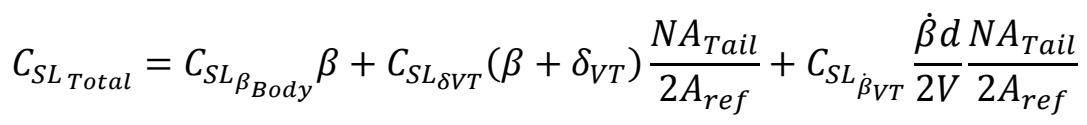

\subsubsection{Yawing Moment}

Similarly, the pitching and yawing moments were symmetric and shown as equations (3-15) through (3-19). However, there was a difference in beta angle being used in this build-up and the beta angle that determined by the 6DoF simulation. This caused the yaw rate to be of opposite sign convention from the beta angle. The actual yaw rate was reversed in sign to correct this issue.

$$
\begin{gathered}
C_{n_{\delta V T}}=\frac{-\eta 2 \pi}{\left(1+\left(\frac{2}{\text { AR }_{\text {Tail }}}\right)\right)} \\
C_{n_{p_{\text {Tail }}}}=\frac{-\left(.6 \eta N A_{\text {tail }} l_{t}^{3}\right)}{A_{\text {ref }} d}\left(\frac{p^{2}}{V^{2}}\right) \\
C_{n_{r_{V T}}}=-2.2 \eta C_{L \text { Side }_{\delta V T}}\left(\frac{\pi}{180}\right) \\
C_{n_{\dot{\beta}_{V T}}}=-2 \eta C_{L \text { side }_{\delta V T}}\left(\frac{\pi}{180}\right)
\end{gathered}
$$




$$
\begin{aligned}
& C_{n_{\text {Total }}}=C_{n_{\beta \text { Body }}}(\beta)+C_{n_{\delta V T}}\left(\frac{\left(\beta+\delta_{V T}\right) \pi}{180}\right)\left(\frac{l_{\text {tail }}}{l_{\text {ref }}}\right) \frac{N A_{\text {Tail }}}{2 A_{\text {ref }}}+C_{n p_{\text {tail }}}(\operatorname{sign}(\beta)) \\
& +\left(C_{n_{r_{V T}}}-C_{n_{\text {r Body }}}\right)\left(\frac{\left(\frac{180}{\pi}\right) r d}{2 V}\right) \frac{N A_{\text {Tail }}}{2 A_{\text {ref }}}\left(\frac{l_{\text {tail }}}{l_{\text {ref }}}\right)^{2} \\
& +C_{m_{\dot{\alpha}_{H T}}}\left(\frac{\dot{\beta} d}{2 V}\right) \frac{N A_{\text {Tail }}}{2 A_{\text {ref }}}\left(\frac{l_{\text {tail }}}{l_{\text {ref }}}\right)^{2}
\end{aligned}
$$

\subsubsection{Rolling Moment}

The presence of tails on a spinning projectile caused a rotational drag to reduce the roll rate (p) of the projectile. Rotational drag was considered when the tail surfaces were non-deflected as well as deflected. The non-deflected tail rolling damping coefficient $\left(C_{l_{p} \text { non-defl. tail }}\right)$ was shown as equation (3-20) (Niskanen 2009). In equation (3-20) non-deflected tail roll damping coefficient was calculated using the root chord $\left(c_{r}\right)$, the tip chord $\left(c_{t}\right)$ and the span of the tails (s). In equations (3-20) and (3-22) the moment arm of the tails about the $\mathrm{x}$-axis $\left(\mathrm{r}_{\mathrm{t}}\right)$ was the projectiles diameter plus half the span of a tail. The deflected tail rolling damping coefficient $\left(C_{l_{p} \text { defl. tail }}\right)$, equation (3-21), was determined by using the lift coefficient from each tail that caused a torque on the body. The combined effect of the deflected and nondeflected tails on the rolling moment coefficient was compiled as equation (3-22).

$$
\begin{gathered}
C_{l_{p} \text { non-defl. tail }}=\frac{-N \eta\left(\frac{2 \pi}{\sqrt{1-M^{2}}}\right)}{A_{\text {ref }} d}\left(\frac{c_{r}+c_{t}}{2} r_{t}^{2} s+\frac{c_{r}+2 c_{t}}{3} r_{t} s^{3}+\frac{c_{r}+3 c_{t}}{12} s^{3}\right)\left(\frac{p}{V}\right) \\
C_{l_{p} \text { defl. tail }}=\frac{2 \pi \eta}{\left(1+\left(2 / A R_{\text {tail }}\right)\right)} \\
C_{l \text { Total }}=C_{l_{p} \text { non-defl. tail }}\left(\frac{p(180 / \pi) d}{2 V}\right)+C_{l_{p} \text { defl.tail }}\left(\frac{\delta \pi}{180}\right)\left(\frac{N A_{\text {tail }}}{A_{\text {ref }}}\right)\left(r_{t}\right)
\end{gathered}
$$

With the total aerodynamic coefficients calculated the forces and moments were determined. The equations of motion were then solved using those aerodynamic forces and moments to model the HP. 


\subsection{Verification of 6DoF model of a HP with Tails}

An experimental projectile was constructed to validate the 6DoF model using the new build-up of aerodynamic coefficients from the tails. In this case, the aerodynamic coefficients and geometry were modified for the values from the experimental projectile. The initial conditions shown throughout this section for the experimental projectile were compiled in Table 3-1; calculated throughout this section, in Appendix C and in Appendix D. The experimental projectile was designed due to its launch system and to ensure stability. The experimental projectile was launched using a 1.5” PVC pipe potato gun style launcher. The fins were designed to be sub-bore so that projectile would fit inside of the pipe to be launched. The inner diameter of the PVC pipe was 1.71" so the projectile's diameter was chosen to be 1.68” (42.67 mm), leaving a small gap so that the projectile did not get stuck in the tube. Stability of this projectile was calculated by first completing a CG analysis and then ensuring that the AC was rearward of the CG. The envisioned design was shown as Figure 3-2. This projectile was built in three sections; nose, body and tail, so components could be added inside of the body and accessed from each end. The three sections were shown by modifying the color of the body section in Figure 3-2. This experimental projectile was equipped with a Px4 autopilot (PX4 Autopilot n.d.), voltage regulator and batteries so that its trajectory could be recorded.

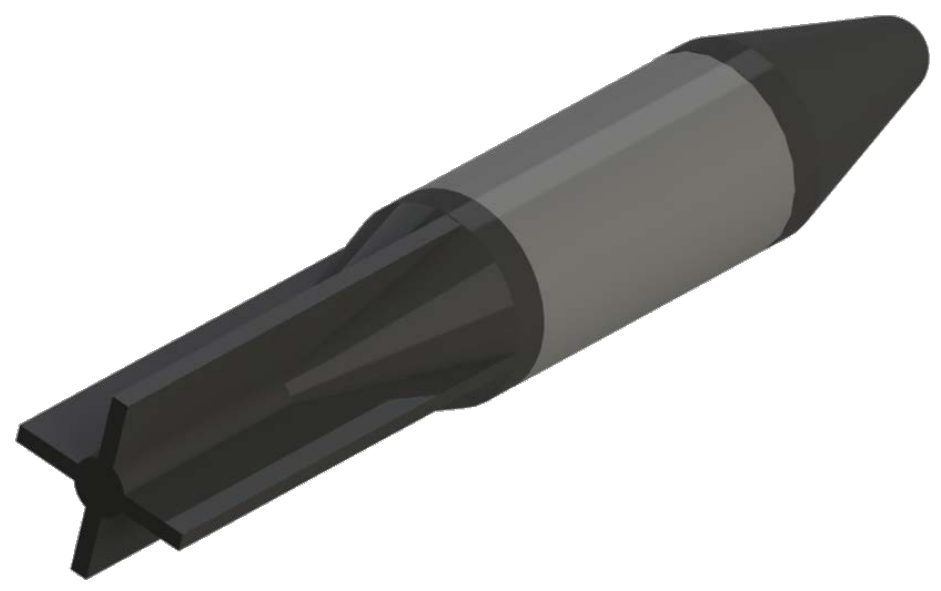

Figure 3-2: Full Projectile with Batteries (Front of Body) and Chip (Rear of Body)

The mass of all the components were measured and the approximate CG location of each component was determined, with the total mass of the projectile being $0.164 \mathrm{~kg}$. The CG location was either measured by hand or estimated with the use of SolidWork's measuring tool. The CG distances were measured from the nose of the projectile. The CG of the test projectile was calculated to be $96 \mathrm{~mm}$ aft of the nose, shown in Appendix C. Meaning that for the projectile to be stable the AC must be greater than $96 \mathrm{~mm}$. 
An aerodynamic build-up of the normal force coefficient was completed on each component and used to determine the AC. The normal force coefficient was calculated using a literature build-up of all components (Barrowman n.d.). Appendix C shows the steps required to determine the normal force coefficient with change in angle of attack $\left(\mathrm{C}_{\mathrm{N}_{\alpha}}\right)$ and the AC of each component. The total normal force coefficient was calculated to be $\mathrm{C}_{\mathrm{N}_{\alpha}}=0.128$ and the AC was determined to be $172 \mathrm{~mm}$ aft of the nose. Resulting in a static margin of $76.5 \mathrm{~mm}$ meaning that the projectile was stable since the AC was aft of the CG.

This test projectile was experimentally tested to determine its range. It was launched

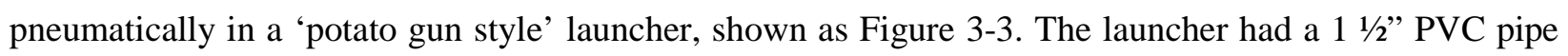
barrel and a 2" PVC pipe pressure chamber. The pressure chamber was connected to an air compressor to the aft end of the pressure chamber and filled. When the red trigger was pulled the air in the pressure chamber went through the valve into the barrel accelerating the projectile.

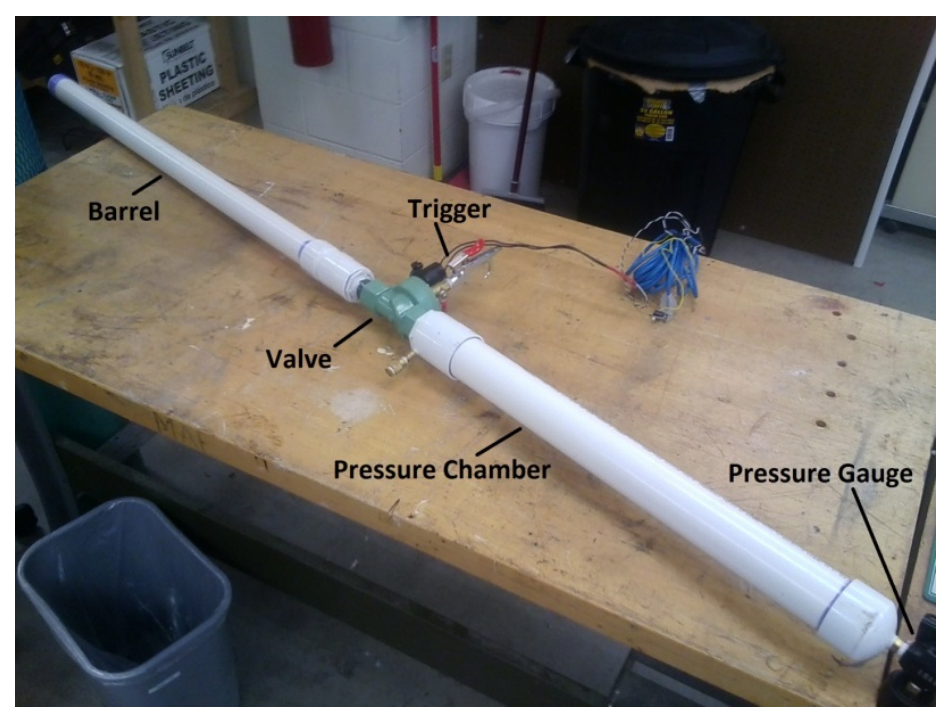

Figure 3-3: Launch System Diagram

The Px4 Autopilot was used to track the orientation and position of the projectile. Setup for the Px4 Autopilot was shown in Appendix D and was used to retrieve the flight data. From the GPS launch data the projectile was seen to have travelled 144 meters. The top view of the projectiles flight was shown in Figure 3-4. The GPS data was converted from Latitude and Longitude to meters then zeroed at the position where the autopilot was turned on using the code from Appendix F. 


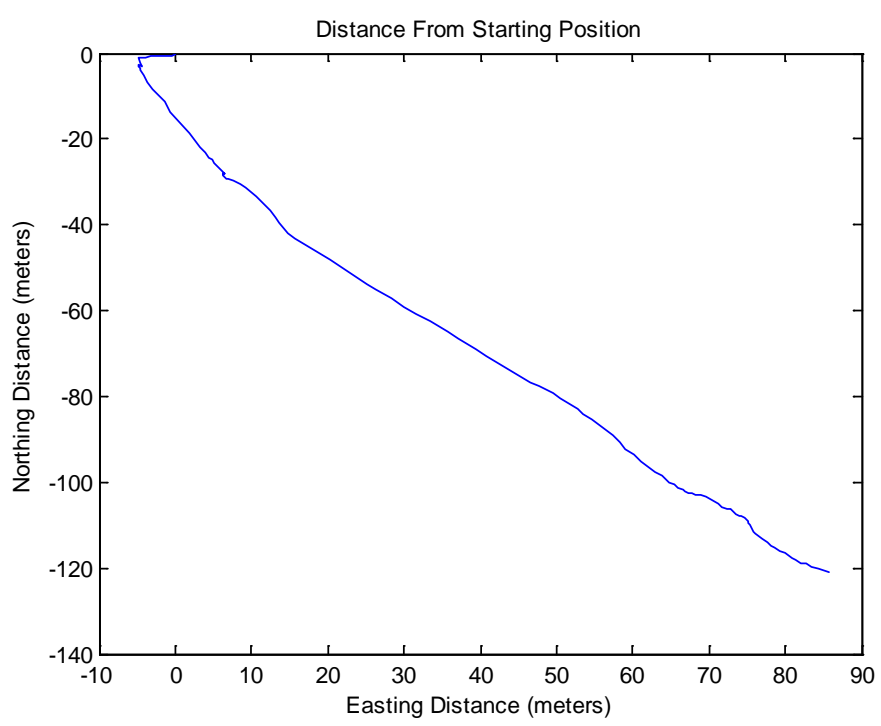

Figure 3-4: Test Projectile Launch, Zeroed from Starting Location

During launch testing the pressure in the chamber was 100 psi which correlated to a launch velocity of between 40 and $45 \mathrm{~m} / \mathrm{sec}$. This correlation of chamber pressure to muzzle was determined experimentally by testing multiple pressures and determining the launch velocity using a high speed camera. Inclination of the launcher was set to approximately 30 degrees. Approximate ranges of launch velocity and inclination were simulated to show an envelope of possible trajectories. The flight model built in this section was used to simulate the same launch and the results were compared. The initial conditions and projectile specifications used in both the flight experiment and simulation are compiled in Table 3-1. Aerodynamic coefficients were calculated in Appendix D. Simulation results in Figure 3-5 show the minimum and maximum range trajectories due to changing the launch velocity and elevation. After simulating all combinations of launch velocity and inclination from the ranges of values in Table $3-1$, the minimum range resulted from the $40 \mathrm{~m} / \mathrm{sec}$ at 28 degree launch and the maximum range was from the $45 \mathrm{~m} / \mathrm{sec}$ at 32 degrees. All other launch angles and velocities fell within that range. When the average value of launch angle and velocity, $42.5 \mathrm{~m} / \mathrm{sec}$ at 30 degrees, was simulation the range was 140.2 meters. Due to the lack of exact values for experimental launch velocity and angle the experimental results were not a good verification of the accuracy of the model. From Figure 3-5 it was noticed that the range calculated by the model was up to 23 meters (16\%) off of the experimental results. With more accurate launch conditions the range of simulation data could be reduced and the error could be found. 
Table 3-1: Simulation Constants for the Experimental Projectile

\begin{tabular}{|c|c|l|}
\hline Launch Velocity & {$[40-45]$} & $\mathrm{m} / \mathrm{sec}$ \\
\hline Launch Elevation & {$[28-32]$} & degrees \\
\hline mass & 0.164 & $\mathrm{~kg}$ \\
\hline$d$ & 0.04267 & $\mathrm{~m}$ \\
\hline$X_{A C}$ & 0.172 & $\mathrm{~m}$ \\
\hline$X_{C G}$ & 0.096 & $\mathrm{~m}$ \\
\hline$I_{x x}$ & $5.36 \times 10^{-4}$ & $\mathrm{~kg} \mathrm{~m}{ }^{2}$ \\
\hline$I_{y y}$ and $I_{z z}$ & $6.07144 \times 10^{-4}$ & $\mathrm{~kg} \mathrm{~m}{ }^{2}$ \\
\hline$C_{L_{\alpha}}$ and $C_{S L_{\beta}}$ & 0.128 & \\
\hline$C_{m_{\alpha_{C G}} \text { and } C_{n_{\beta}}}$ & -0.098 & \\
\hline$C_{D}$ & 0.24 & \\
\hline$C_{m_{q}}$ and $C_{n_{r}}$ & -0.1 & \\
\hline
\end{tabular}

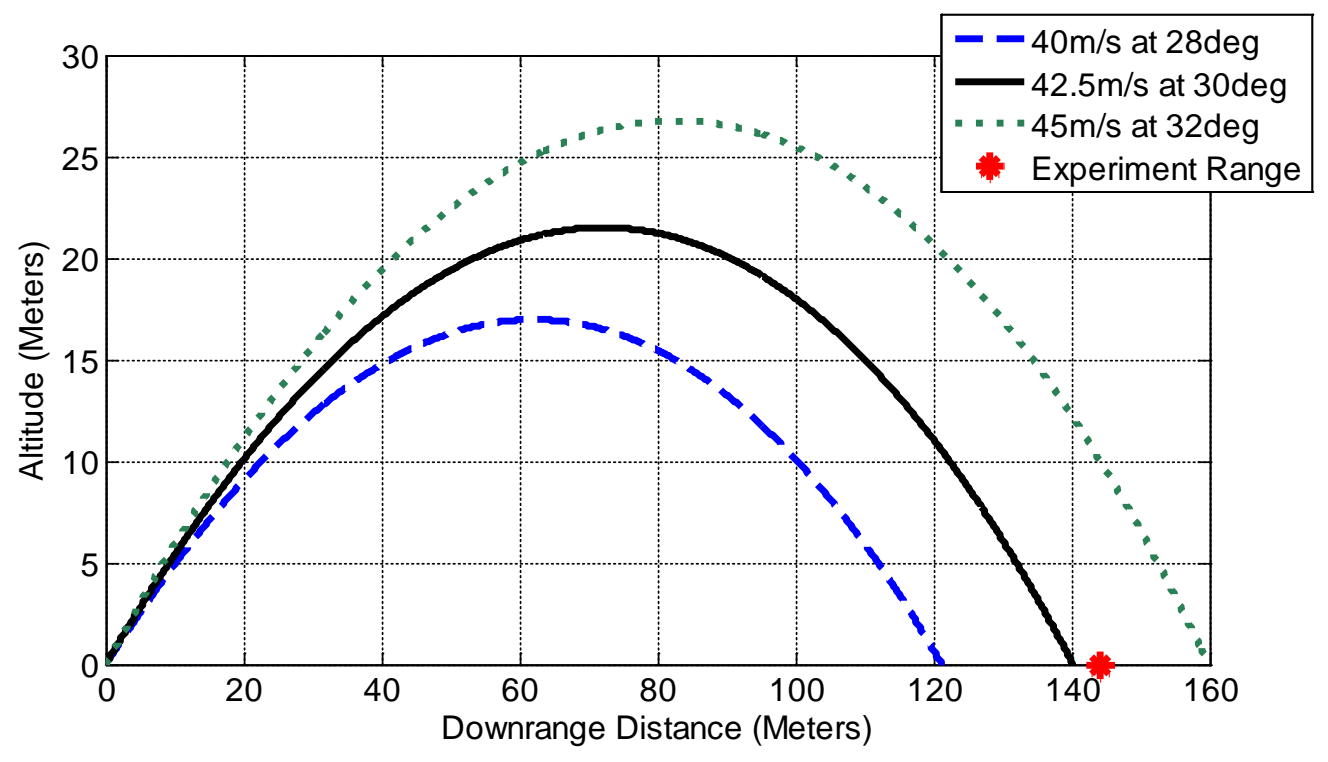

Figure 3-5: Simulation Results of Experimental Test

After verifying simulation results with the experimental test, the new coefficient build-up in model was determined to be useable in this case. A tail controller could now be added to the model and as long as the tail control model was meticulously designed then the model should remain accurate. 


\subsection{Tail Sizing for Static and Dynamic Stability}

Now that tail surface contributions have been added to the 6DoF model, the geometry of the tails could be calculated. The tails on this HP were required to meet two criteria:

1.) The tail area must satisfy static and dynamic stability criteria for the projectile.

2.) The tail surface area must be large enough to ensure that the HP was de-spun to zero degrees per second in less than 2 seconds, without the tails being deflected.

Static stability for the HP was determined by varying the chord and span of the tails to calculate the value of $\mathrm{M}$, equation (2-10). In equation (2-10) $C_{m_{\alpha}}$ was the variable that could be adjusted by adding tails to the projectile, the other values remained constant. In Figure 3-6, the pitching moment coefficient value with respect to tail chord and span was shown. Static stability was established when the pitching moment coefficient was less than zero. The dark red section fills in the positive pitching moment coefficients determined from the tail geometry. Therefore the tail geometry must be chosen from a nondark-red location.

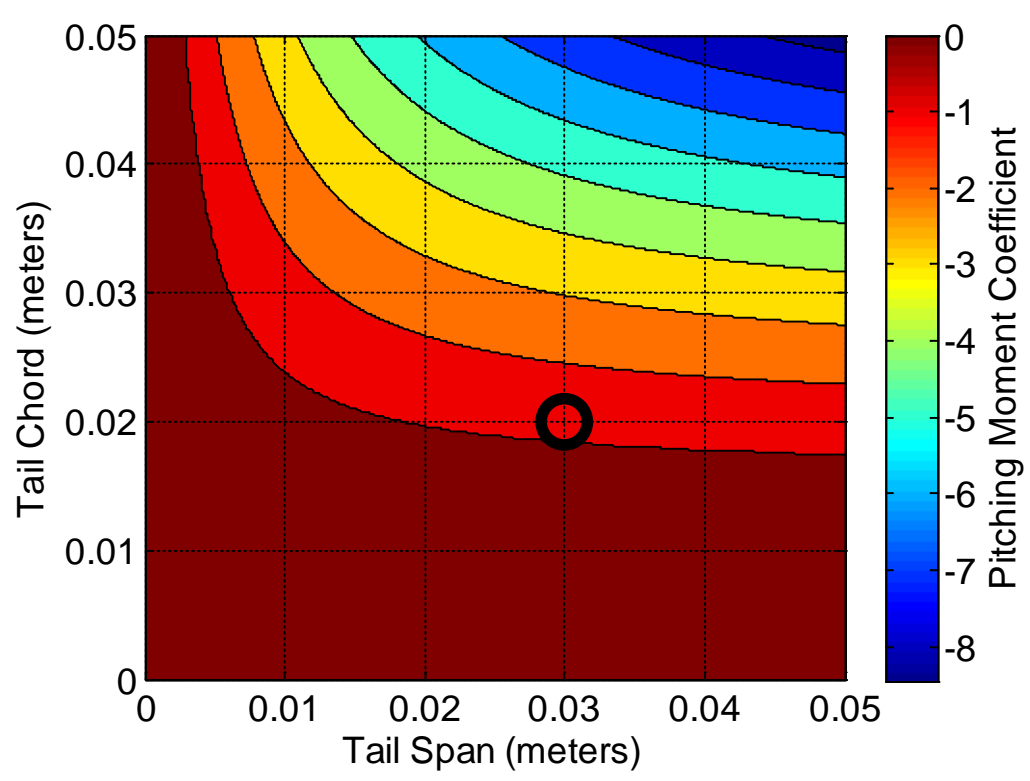

Figure 3-6: Static Stability Examination with respect to Tail Sizing 
The projectile must also be dynamically stable, meaning that the non-dimensionalized dynamic stability factor $(\mathrm{H})$ from equation (2-5) must be positive. A plot similar to the static stability plot was created to show the $\mathrm{H}$ value with respect to the tail sizing. In Figure 3-7, it was noticed that for all tail sizes investigated the $\mathrm{H}$ value was a positive value, meaning any choice in tail geometry from Figure 3-7 yielded a dynamically stable projectile. However, the values get smaller, less dynamically stable, as they move towards the upper-right corner of the figure. So the tail geometry was chosen from a point on Figure 3-6 which yielded a negative pitching moment coefficient and as close to the bottom left corner in Figure 3-7.

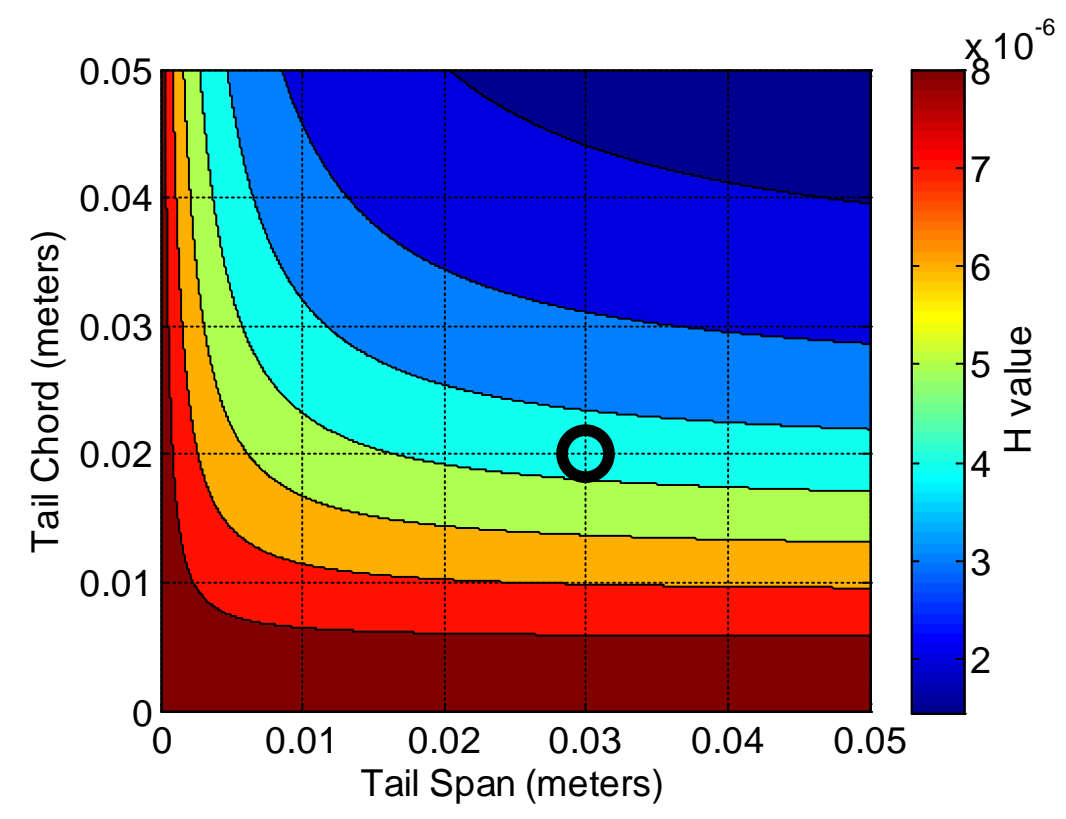

Figure 3-7: Dynamic Stability Examination with respect to Tail Sizing

The tail area required to satisfy the criteria of de-spinning in less than two seconds without tail deflection was determined by calculating the angular acceleration of the projectile due to the presence of the tails, using equation (3-23). The roll rate was calculated using a discrete time step then used to solve the rolling acceleration $(\dot{p})$ for the next time step. In this equation, the effective angle of attack $\left(\alpha_{e f f}\right)$ was multiplied by lift coefficient created for a tail on all four tails. The effective angle of attack was the angle created by freestream velocity and the roll rate (p) as seen in Figure 3-8. They were then multiplied by the moment arm of each tail $\left(\mathrm{r}_{\mathrm{t}}\right)$ which was considered as the mid span point. As angular acceleration in the roll axis changes the angular velocity over time, the effective angle of attack of the tails decreases thus reducing the lifting force on the tails. In equation (3-23), the values that could be modified were the span and the tail area. By varying these two parameters the roll rate was calculated over time until it reached zero degrees per second. A table of de-spin times for different tail configurations can be seen in Table 3-2. The de-spin time in this table were based on the roll rate going below 0.01 degrees per second. The 
de-spin time table shows that the tail requirements for de-spin are, at least, an area of $600 \mathrm{~mm}^{2}$ and a span of $30 \mathrm{~mm}$.

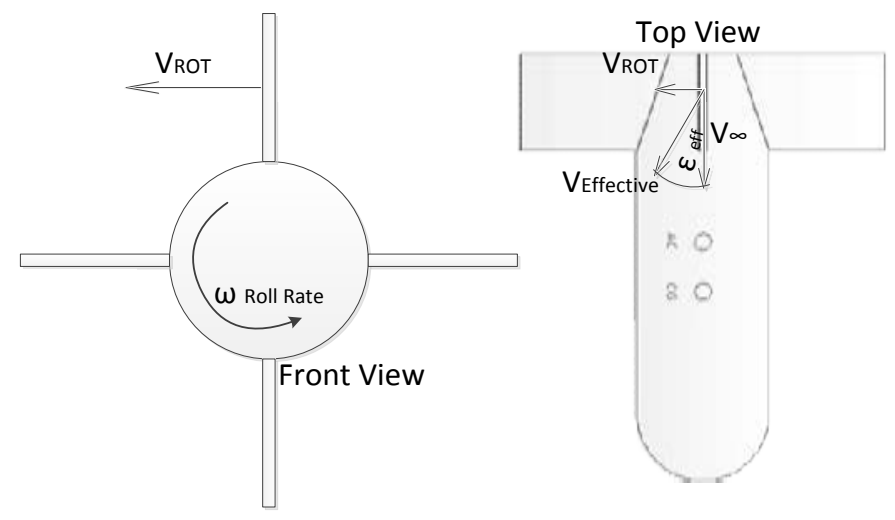

Figure 3-8: Effective Angle of Attack on tails from Roll Rate

$$
\dot{p}=\frac{\tau}{\mathrm{I}_{\mathrm{xx}}}=\frac{-2 \bar{q} A_{r e f} C_{L_{\delta H T}} \alpha_{e f f} r_{t}}{I_{x x}}=\frac{-.5 \rho \mathrm{N} \mathrm{A}_{\mathrm{tail}} \mathrm{V}^{2} \mathrm{r}_{\mathrm{t}} \frac{2 \pi}{1+2 /\left(\mathrm{s}^{2} / \mathrm{A}_{\mathrm{tail}}\right)} \tan ^{-1} \frac{\mathrm{p}}{\mathrm{V}} \mathrm{r}_{\mathrm{t}}}{\left(5.4412 \times 10^{-5}\right)}
$$

Table 3-2: De-spin Time Based on Tail Geometry

\begin{tabular}{|r|r|r|r|}
\hline Span (mm) & Chord (mm) & Tail Area $\mathbf{( m m}^{\mathbf{2}}$ ) & Time (sec) \\
\hline 20 & 20 & 400 & 4.41 \\
\hline 20 & 25 & 500 & 4.11 \\
\hline 20 & 30 & 600 & 3.91 \\
\hline 20 & 35 & 700 & 3.77 \\
\hline 20 & 40 & 800 & 3.66 \\
\hline 25 & 20 & 500 & 2.58 \\
\hline 25 & 25 & 625 & 2.38 \\
\hline 25 & 30 & 750 & 2.24 \\
\hline 25 & 35 & 875 & 2.14 \\
\hline 25 & 40 & 1000 & 2.07 \\
\hline 30 & 20 & 600 & 1.64 \\
\hline 30 & 25 & 750 & 1.5 \\
\hline 30 & 30 & 900 & 1.4 \\
\hline 30 & 35 & 1050 & 1.33 \\
\hline 30 & 40 & 1200 & 1.28 \\
\hline
\end{tabular}

From Table 3-2, it was seen that many different combinations of tail chord and span will allow for de-spin time of less than 2 seconds, but the smallest tail size of $600 \mathrm{~mm}^{2}$ was used as the required tail area for de-spin. The smallest tail area was chosen to reduce the drag contribution from the tails, since skin friction drag was proportional to the wetted area. The de-spin times from Table 3-2w ere similar to the values determined by CFD and numerical flight testing for a $40 \mathrm{~mm}$ HP design (Vogel 2012). 
The tails were not considered to have affected the moment of inertia for the projectile since a structural design was not yet done. However, the changes that could occur from the change in CG, AC and $I_{x x}$ were examined to determine if this assumption would minimally effect de-spin and stability criteria. From the calculations shown in Appendix B, if carbon fiber tails were used, the CG would be shifted aft by $4 \%$ of the projectiles diameter while the AC was shifted aft $264 \%$ of the projectile diameters. This resulted in a 5\% difference in SM when tails were and were not considered to affect CG allowing mass to be ignored in this case.

In Appendix B, the change in $\mathrm{I}_{\mathrm{xx}}$ due to the tails being added to the projectile was considered. The de-spin time was seen to be increased by $2 \%$ of the total flight time due to the increased moment of inertia. The moment of inertia should be calculated when the actual design is built but was ignored since mass properties could be manipulated in this theoretical method.

Now, with both requirements met, the tail geometry was determined. From the two above conditions the tail area must be $600 \mathrm{~mm}^{2}$ so the span must be $30 \mathrm{~mm}$. This means that the chord of the tail was $20 \mathrm{~mm}$. The tail surfaces were designed as rectangles so the root chord and tip chord were $20 \mathrm{~mm}$. The tail geometry was chose as four, $20 \mathrm{~mm}$ by $30 \mathrm{~mm}$, tails. This tail geometry yields a pitching moment coefficient value of -0.0266 . This geometry, shown on the modified M781 in Figure 3-9, yielded a statically and dynamically stable projectile.

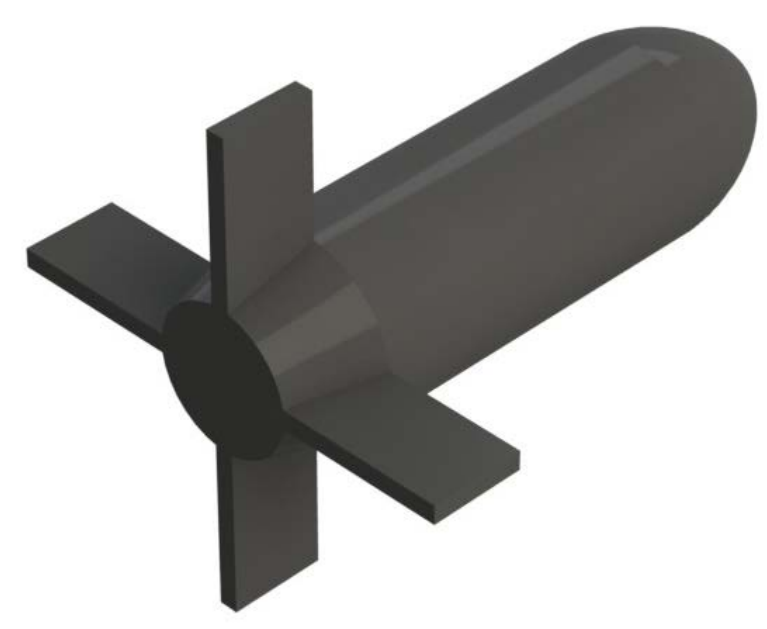

Figure 3-9: Modified M781 with Four Tails 


\section{Chapter 4 Tail Control for Guidance}

Tail control was added to the flight model of the HP with the use of a tail controller sub-system. The tail controller sub-system, shown in Figure 4-1 as left most block, was used to calculate the required tail deflections to de-spin, roll-level and guide the HP to a desired point of impact. The tail controller submodel had a "states" input that used rotational angle, rotational velocity and translational velocity which were received from the '6DoF (Euler Angles)' block (right-center). An input of the rolling moment coefficient was also important in the switch function, shown later, which was received from the 'Calc Forces’ block (left-center).

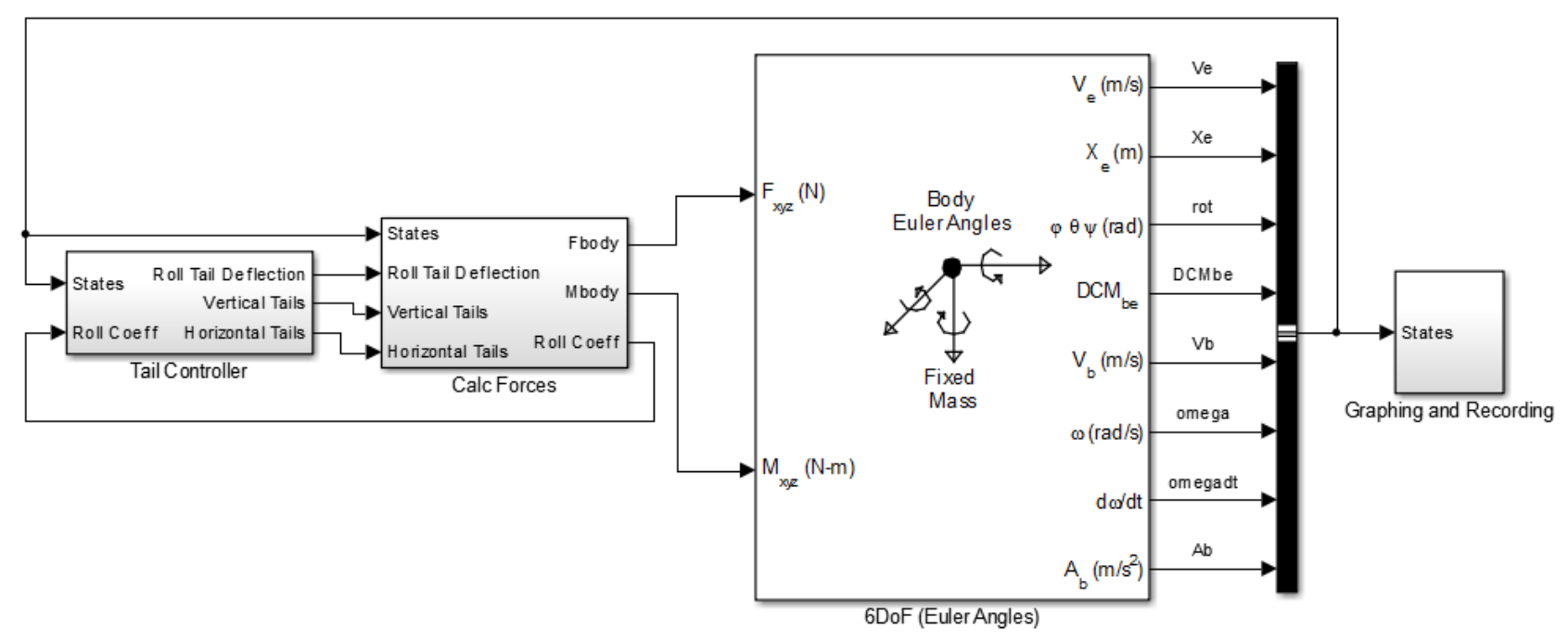

Figure 4-1: Simulation Model with Tail Control

\subsection{Tail Feedback Control using Multiple Controllers}

Figure 4-2 shows the Tail Controller sub-system of Figure 2-5. In this sub-system the tail deflection outputs were broken down into the deflection required for roll, heading and vertical control from top to bottom. In Figure 4-2, each set of tail deflections were determined through separate control methods with a servo delay applied. 


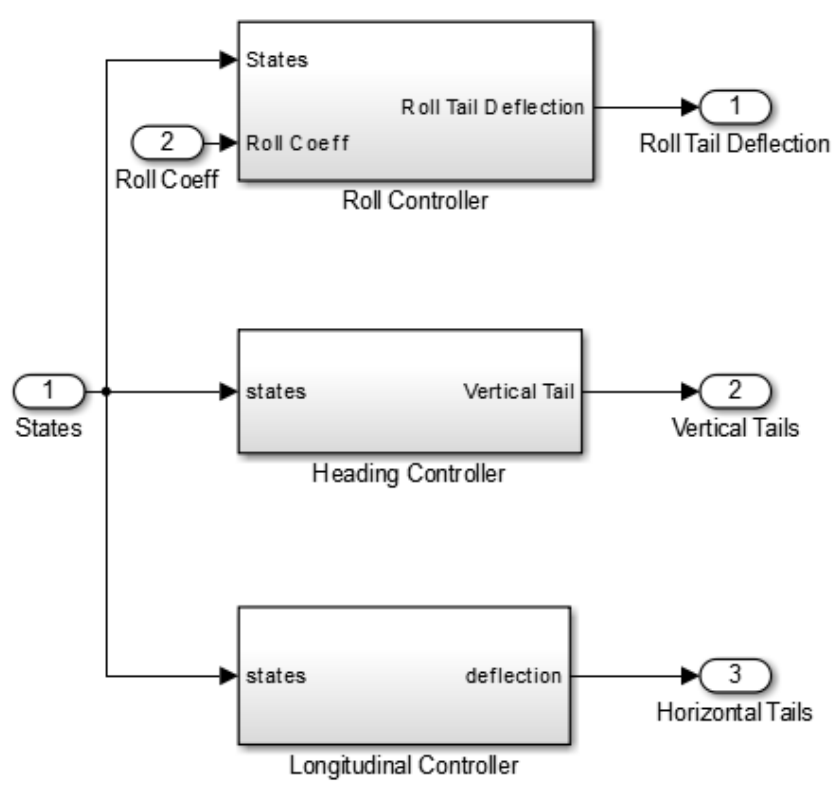

Figure 4-2: Top Level Three Tail Controllers

Three separate control systems were used so that each of the three control modes could be separated. The roll controller was used to deflect all tails in the same direction to produce a rolling torque to the projectile. The heading controller produced a tail deflection on the vertical tails to create a torque on the projectile about the z-axis causing the projectile to yaw. Similar to the heading controller, the vertical controller was used to produce a torque on the projectile about the y-axis with the horizontal tails pitching the projectile. Vertical and horizontal tails deflect as shown in Figure 3-1.

One servo on each tail was used to deflect the tail surface due to the required deflection from the controller. Since the roll controller acts on all four tails and the vertical and heading controllers act on two tails each. The tail command for roll needs to be combined with the vertical and heading command. 


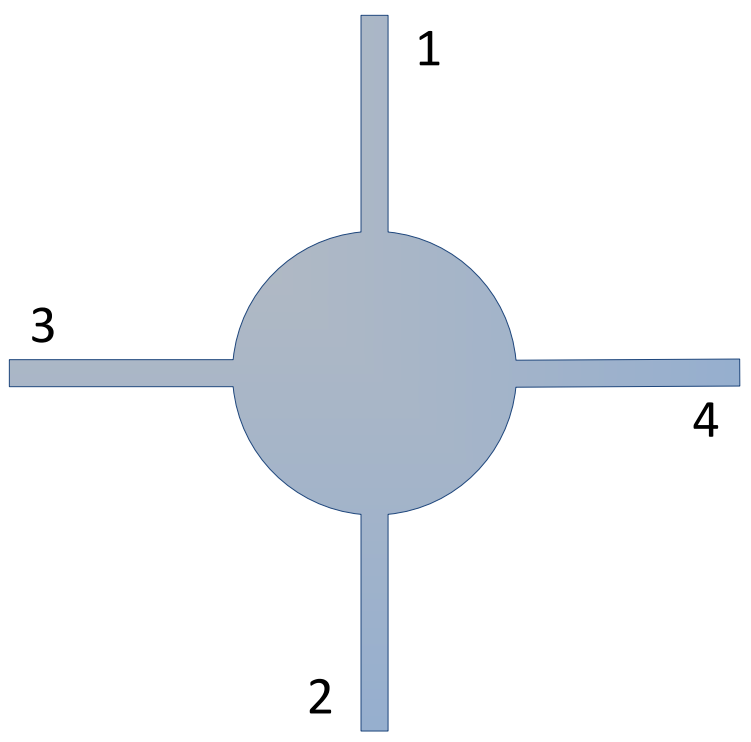

Figure 4-3: Tail Numbering Convention

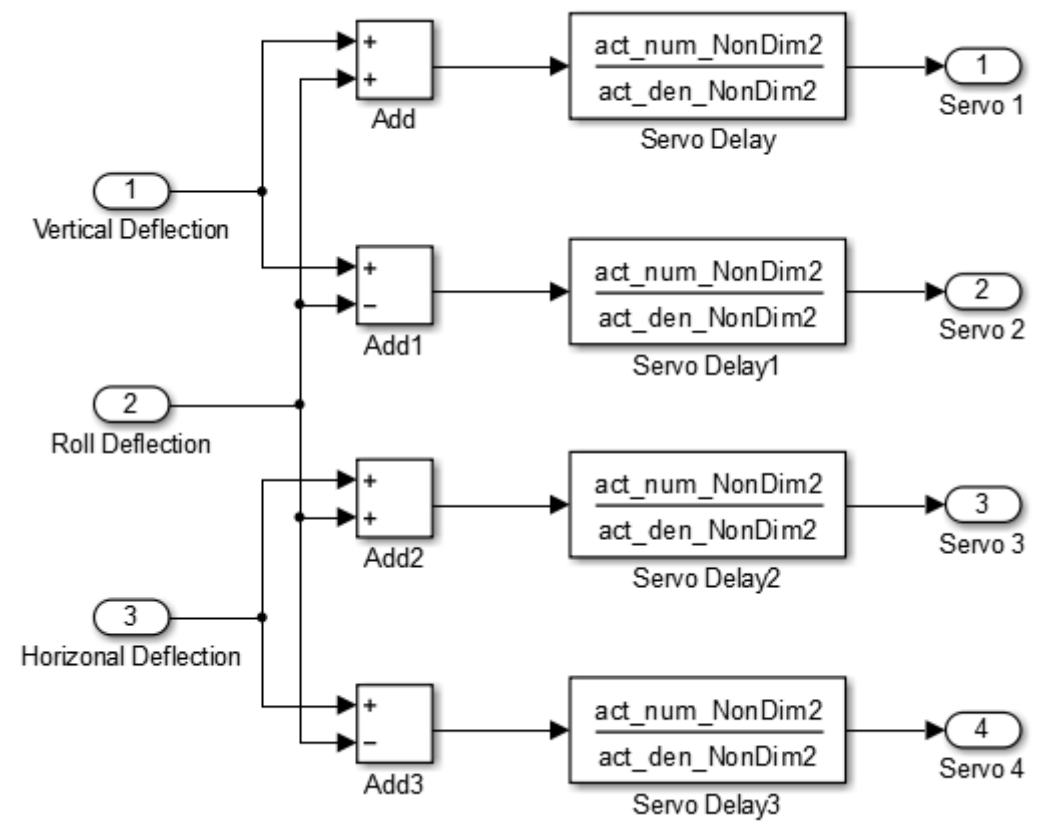

Figure 4-4: Controller Outputs for Servo Inputs

\subsection{Servo Delay Model}

When using an electric servo, there was a delay from the desired output to the actual angle of the servo. An electric servo uses an input of a square wave of a particular pulse width that corresponds to a desired position for the servo arm. The servo arm position could be measured by a change in resistance in the servo. A transfer function was required to model the delay of the servo to make the simulation model 
more realistic. The servo transfer function was applied as the Servo Delay transfer function in Figure 4-2. All three Servo Delay transfer functions were the same for the roll, heading and longitudinal controllers.

The servo delay transfer function was determined by logging the position potential from a servo due to a square wave input. The servo was deflected 24 times and an average of the voltage out was calculated then converted to degrees. Using Matlab’s system identification toolbox a transfer function was estimated with two poles and one zero. The transfer function estimated as the servo delay model was shown as equation (4-1). The actual response and estimated response were shown in Figure 4-5, where the servo delay transfer function was due to a unit step input at zero seconds. There was a difference in the actual response and the transfer function representation because the actual response was a built-in function of the servo, that was unknown, and was estimated using a second order transfer function.

$$
\text { Servo Delay } T F=\frac{-1.3479 s+773.7087}{s^{2}+42.8113 s+773.4382}
$$

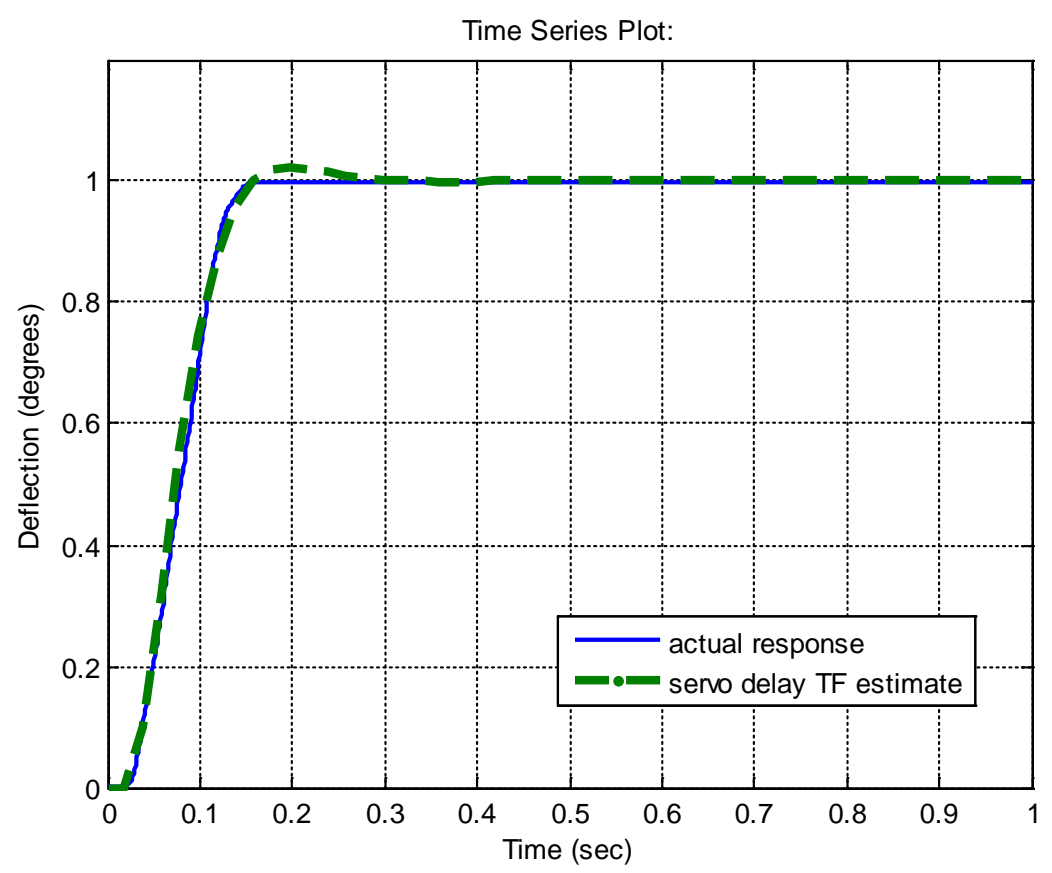

Figure 4-5: Servo Delay Response 


\subsection{Roll Control}

The tail controller sub-model, Figure 4-2, was created to make the required tail deflections to despin and roll-level the projectile. It was determined that two separate controllers would be required to despin and roll-level the projectile, and then a transition between these two controllers was needed. The roll rate (de-spin) controller was designed by having a PID controller that drives the roll rate to zero degrees per second. The roll position (roll-leveling) controller was designed as a PID controller that drives the absolute roll position to zero degrees. A PID controller was used because of the combined effects of having a proportional, derivative and integral controller found in Matlab/Simulink product help (Matlab 2014), as seen in Section 2.7. The PID controller was found in the "Continuous" sub-tab of the “Simulink” tab in the Simulink Library Browser. Each PID controller runs at a continuous-time domain with a filter coefficient $(\mathrm{N})$ of $\mathrm{N}=100$.

Each of the four tails had an adjustable local angle of attack that was used to create a normal force, which caused a rolling torque on the projectile. The tail deflections were limited to 10 degrees in each direction so that flow separation was not considered. The 10 degree limit of tail deflection was achieved in the model through the PID advanced tab of the PID controller with a 10 degree and -10 degree saturation limit. A de-spin switch function and controller switch was used to switch between to two controllers. Tuning of the PID's and determining the switch function were very critical and are explained later. A tenth of a second delay was used to allow for tube perturbations to be damped out and give time for tail surfaces to be deployed.

Figure 4-6 was the roll controller sub-model that had inputs of rotation angles (rot), rotation rates (omega) and earth referenced velocity $\left(\mathrm{V}_{\mathrm{e}}\right)$. In Figure 4-6 the upper path was the roll-leveling PID controller, the middle path was the roll rate controller and the lower path was used for the switch function. The controller switch chooses the controller in which the actual tail deflection comes from, due to the despin switch function. 


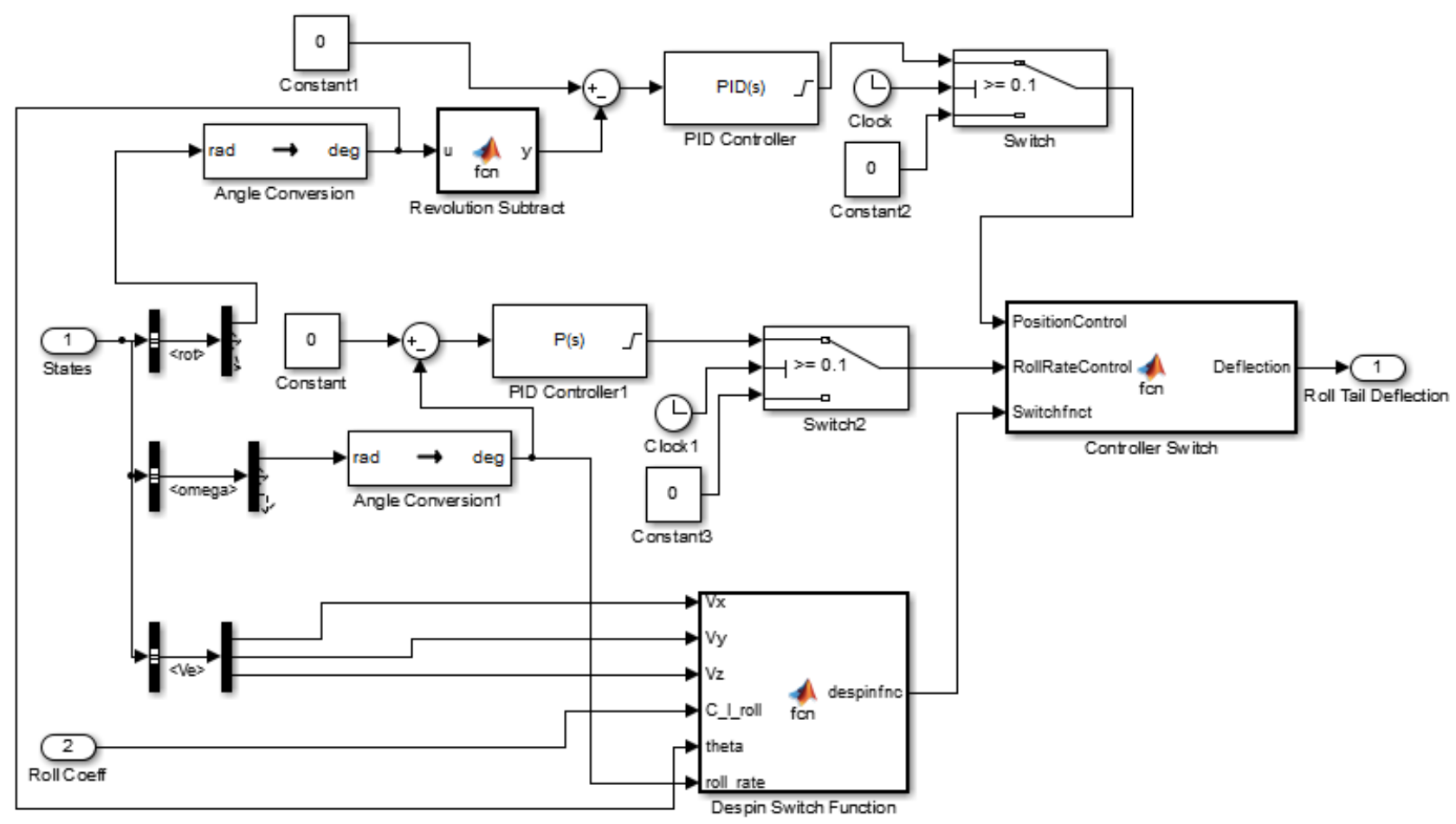

Figure 4-6: Roll Controller Sub-model

\subsubsection{Tuning Dual Mode Roll Controller}

The method that was used to tune these PID controllers of the roll control system was a trial and error technique where all gain values were initially set to zero. One gain value was changed at a time by running multiple values for that particular gain and analyzing the responses. Once that gain value was determined it was saved and then the next gain value was evaluated. The time of flight was shown as the percentage of flight time because different projectiles, with a different total flight time, could use the same method to be de-spun and roll-leveled.

The first gain value that was determined was the proportional gain for the de-spin controller. The gain value for the de-spin controller was determined by disconnecting the position controller from the controller switch block and having the initial roll rate set at $62.5 \mathrm{~Hz}$ (Cooke 2010). The gain value was tuned by determining which gain value gave the quickest roll rate error reduction. The quickest roll rate error response was chosen as $\mathrm{p}_{\text {roll }}=0.0025$ because after testing two gain values the response of roll rate error goes to zero degrees per second quickest. The roll rate error was seen in Figure 4-7, where $\mathrm{p}_{\text {roll }}=0.0025$ yielded the fastest settling time. The roll rate error goes to zero degrees per second and had no steady state error, so no integral gain was needed for this controller. Also, the derivative gain value was set at zero since there was no overshoot criterion and the positioning controller takes over when the roll rate was in a range closer to zero degrees per second. 


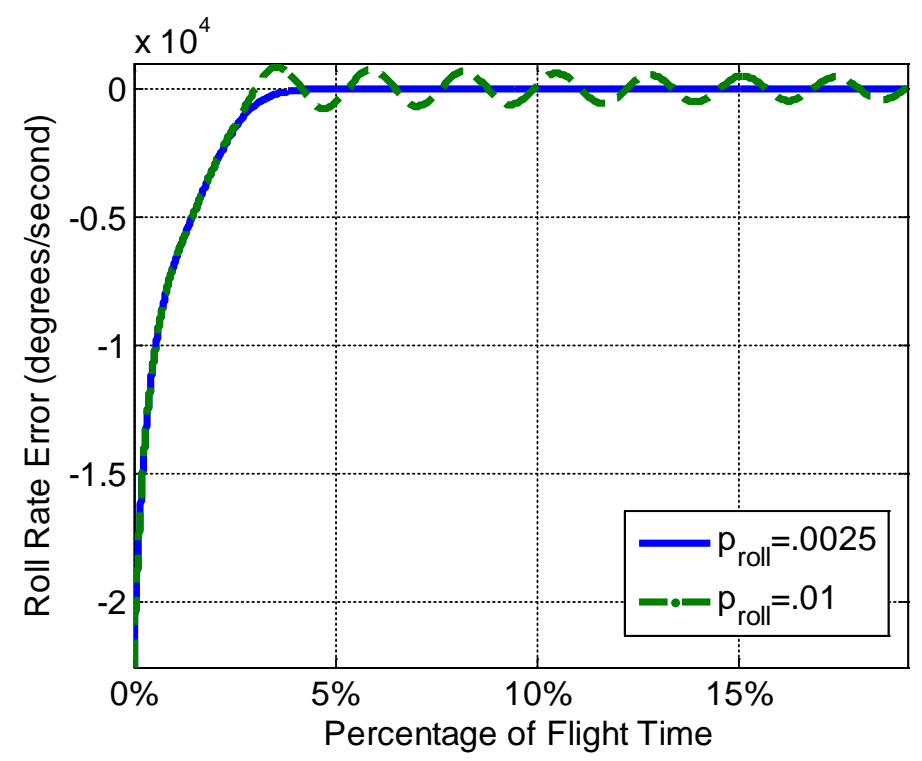

Figure 4-7: Roll Rate Error with Changing Proportional Gain

The gain values that were used in the roll position controller were determined by starting with the projectile at 180 degrees off the roll-level position and no rotational rate. The roll-leveling controller, which was disconnected from the controller switch block for the roll rate controller gain value test, must be reconnected to the controller switch for tail deflection, shown in Figure 4-6. An iterative process was used since more than one gain value needed to be tuned. A proportional gain of 0.01 was assumed first to begin the process. The desired response to the roll angle error plot shown in Figure 4-8 is the fastest first order response. 


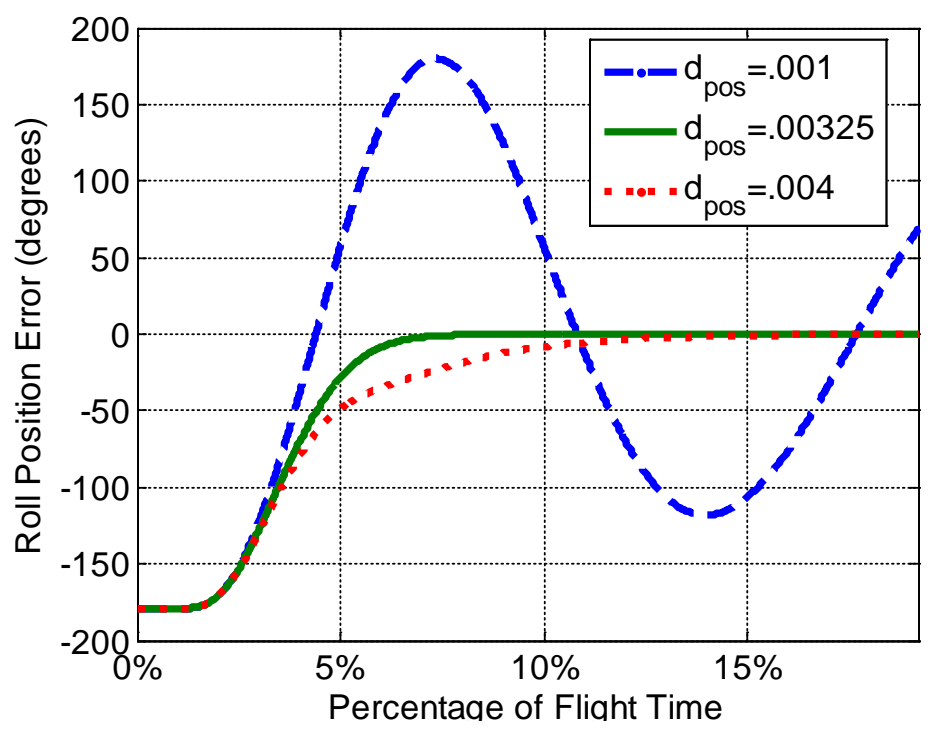

Figure 4-8: Position Error with Changing Derivative Gain

The derivative gain which yielded the desired response in Figure 4-8 value and was used in the next iteration was $d_{p o s}=0.00325$. The reason why this gain value was kept for the next iteration was that it did not overshoot zero degrees and it reached zero degrees faster than having the gain set as 0.004 . Now that a derivative gain has been chosen the proportional gain that was assumed in the first iteration must be reevaluated.

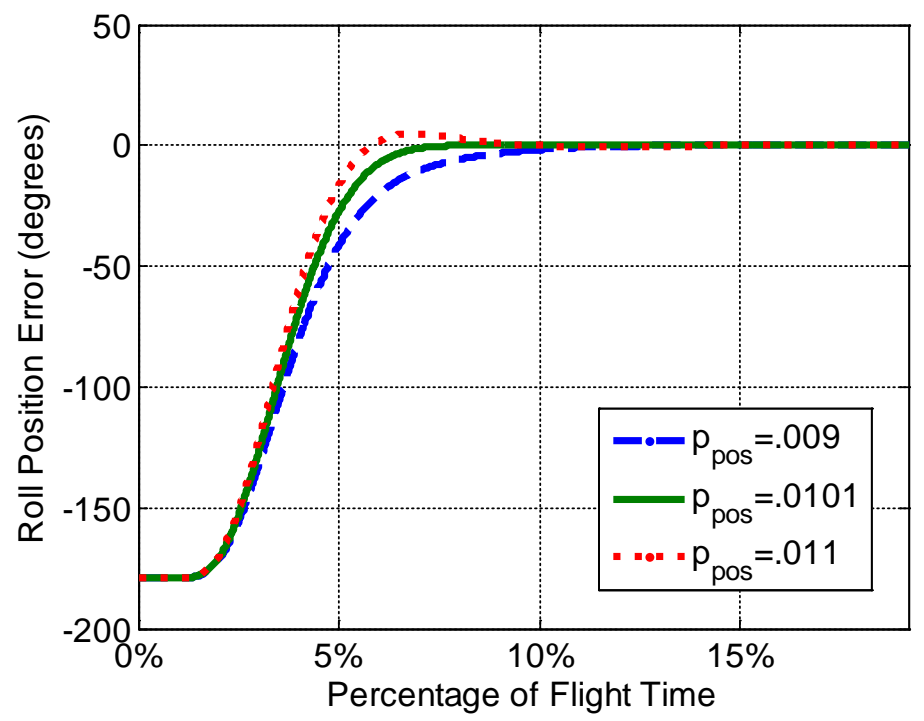

Figure 4-9: Roll Position Error with Changing Proportional Gain 
The proportion gain was found to be $\mathrm{p}_{\mathrm{pos}}=0.0101$, as shown in Figure 4-9. There was no need to change the integral gain value in this PID controller since the response went to zero, meaning there was no steady state error. The final iteration of this process was to check ti see if a fine-tune adjustment was needed for the derivative gain with the new proportional gain value. The derivative gain remained unchanged as $d_{\text {pos }}=0.00325$ after viewing the response of Figure $4-10$, which yielded the fastest settling time.

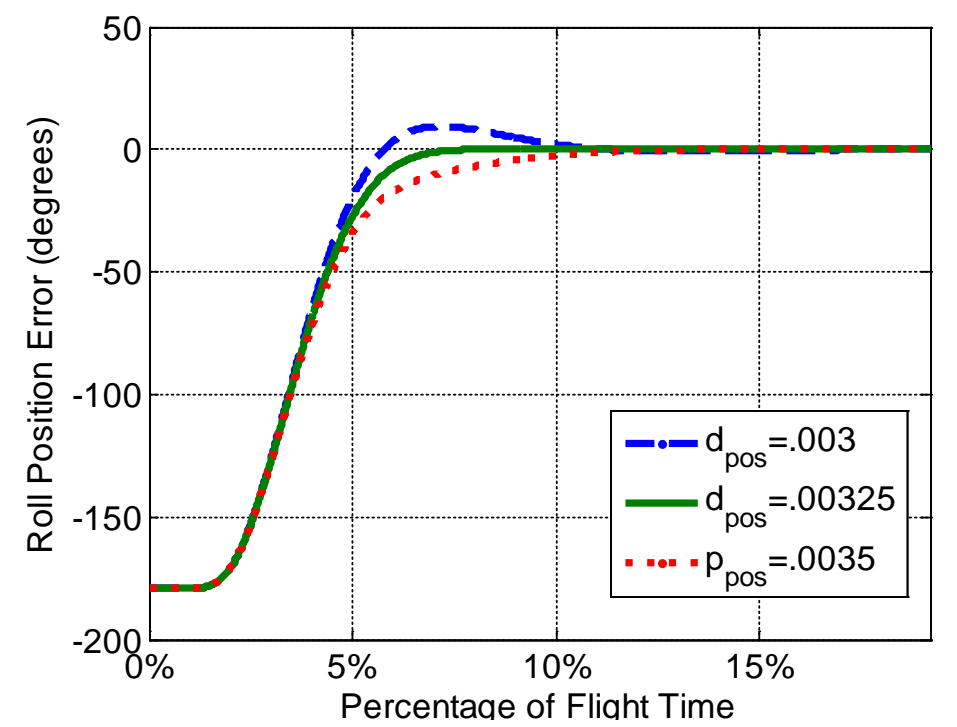

Figure 4-10: Roll Position Error with Fine-tuning Derivative Gain

\subsubsection{Determine Transition Point between Two Controllers}

With the gain values determined for the de-spin and roll-leveling controllers it was important to determine the point at which the actual tail response was changed from the roll rate controller to the rollleveling controller. This switch must be adaptable to different conditions since the initial roll position leaving the tube was random. A switch was created between the two controllers to choose the output that was desired. This switch was used to determine that; with the torque created by the tails the projectile will be able to rotate at its current angular velocity and stop at its roll level position with no tail deflection. This switch function was calculated as equation (4-2). Where $\Delta \varphi$ was the difference in the actual roll angle and the zero degree level roll angle. When the switch factor drops below zero the Controller Switch Block, of the roll controller sub-model, changed the actual response of the tail deflections from the response of the roll rate controller to the response of the roll-leveling controller.

$$
\text { Switch Factor }=\frac{.5 \rho V^{2} A_{\text {ref }} d C_{l \text { Total }}}{I_{x x}}+\frac{p^{2}}{\Delta \phi}
$$




\subsubsection{Roll Controller Results}

In this case for the $40 \mathrm{~mm}$ HP the tail geometry was determined to be for $30 \mathrm{~mm}$ span by $20 \mathrm{~mm}$ chord. With the tails sized and the dual-mode roll PID feedback loops created, the proportional gain value was determined for the de-spin controller to be 0.0025 . The proportional and derivative gains for the position controller were determined to be 0.0101 and 0.00325 respectively. The integral and derivative gain values for the de-spin controller ( $i_{\text {roll }}$ and $d_{\text {roll }}$, respectively) and the integral gain value of the roll leveling controller $\left(i_{\text {pos }}\right)$ were set to zero and compiled in Table 4-1. With the transition switch, the $40 \mathrm{~mm}$ HP was de-spun and roll-leveled in 5\% of its flight time. The results of the tail geometry as well as the despin and roll-leveling performance was compiled in Table 4-2.

Table 4-1: Compilation of Gain Values from De-spin and Level Controller

\begin{tabular}{|r|l|}
\hline Gain Term & Gain Value \\
\hline $\mathrm{p}_{\text {roll }}$ & 0.0025 \\
\hline $\mathrm{i}_{\text {roll }}$ & 0.0 \\
\hline $\mathrm{d}_{\text {roll }}$ & 0.0 \\
\hline $\mathrm{p}_{\text {pos }}$ & 0.0101 \\
\hline $\mathrm{i}_{\text {pos }}$ & 0.0 \\
\hline $\mathrm{d}_{\text {pos }}$ & 0.00325 \\
\hline
\end{tabular}

Table 4-2: Tail Geometry and Performance Results

\begin{tabular}{|r|l|}
\hline$A_{\text {tail }}$ & $600 \mathrm{~mm}^{2}$ \\
\hline $\mathrm{S}$ & $30 \mathrm{~mm}$ \\
\hline $\mathrm{C}_{\mathrm{r}}$ & $20 \mathrm{~mm}$ \\
\hline $\mathrm{C}_{\mathrm{t}}$ & $20 \mathrm{~mm}$ \\
\hline $\mathrm{N}$ & 4 \\
\hline De-spin Time & $3 \%$ \\
\hline Roll Leveling Time & $5 \%$ \\
\hline
\end{tabular}

Results of the roll-leveling controller were shown in Figure 4-11 where the roll angle was leveling to zero degrees in 5\% of the flight time. In Figure 4-11, the roll position never goes above 180 degrees or below -180 degrees because as the projectile rolls past 180 degrees it resets back to -180 degrees. Figure 4-12 shows the results of the de-spin controller where the roll rate decreased to zero degrees per second in $3 \%$ of the flight time. 


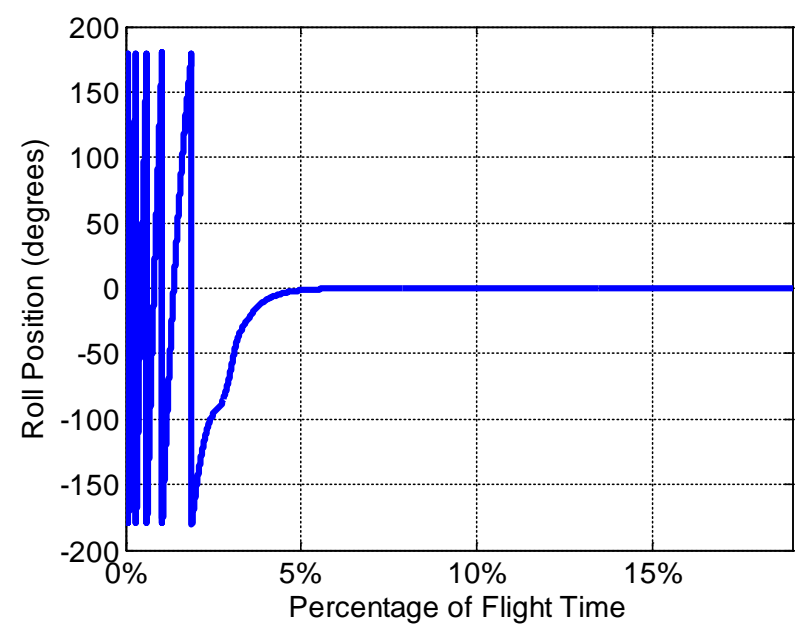

Figure 4-11: Roll Angle through first 20\% of Flight

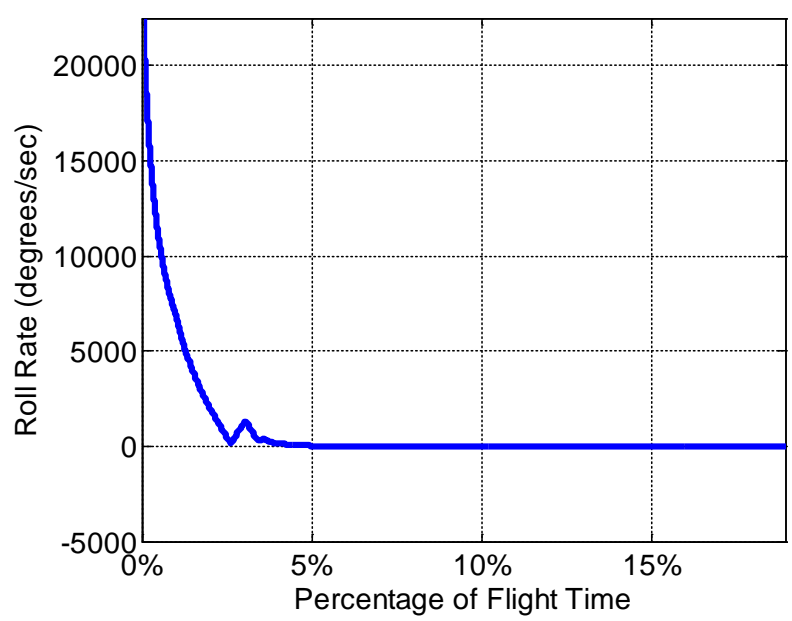

Figure 4-12: Roll Rate through first $20 \%$ of Flight

While de-spinning and roll-leveling, the projectiles overall trajectory was minimally changed from that of the baseline $40 \mathrm{~mm}$ M781. Figure 4-13 and Figure 4-14 show the altitude and distance, respectively, of the flight paths for the M781 projectile and the HP that was configured throughout this process. From Figure 4-13 it was shown that there was a 3.1\% reduction of altitude and in Figure 4-14 a $3.2 \%$ reduction in distance was seen, when launched at a 50 degree elevation. The reduction of distance and altitude were due to the additional drag from the tails. The range and altitude reduction results were compared with projectile motion equations with drag in Appendix D where the range was seen to be decreased by $1.9 \%$ and the maximum altitude was seen to be reduced by $1.1 \%$. 


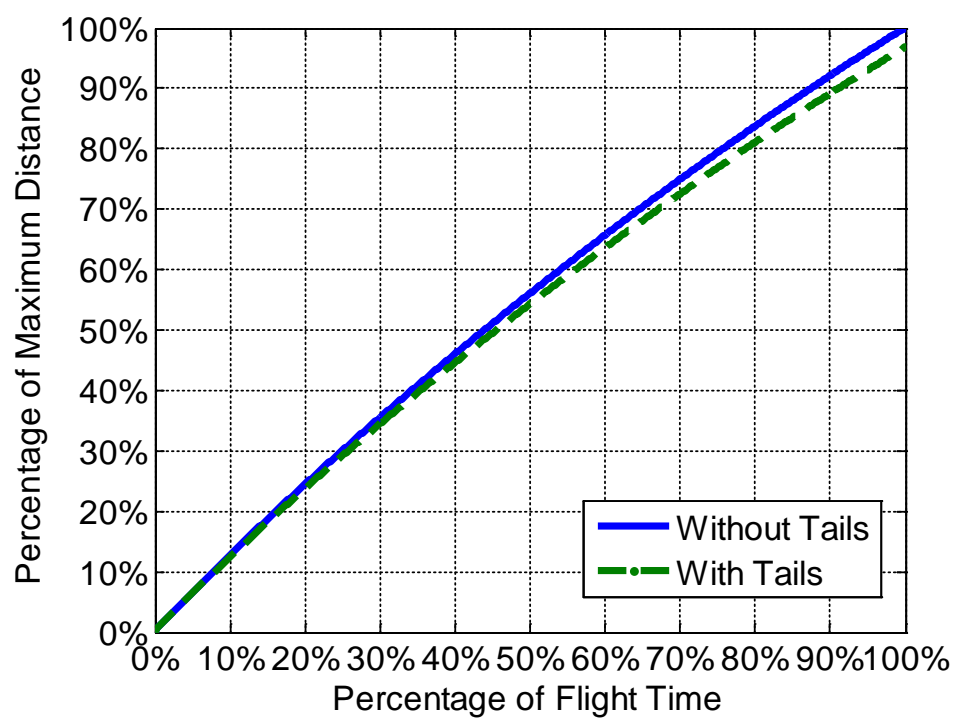

Figure 4-13: X-directional Distance Traveled

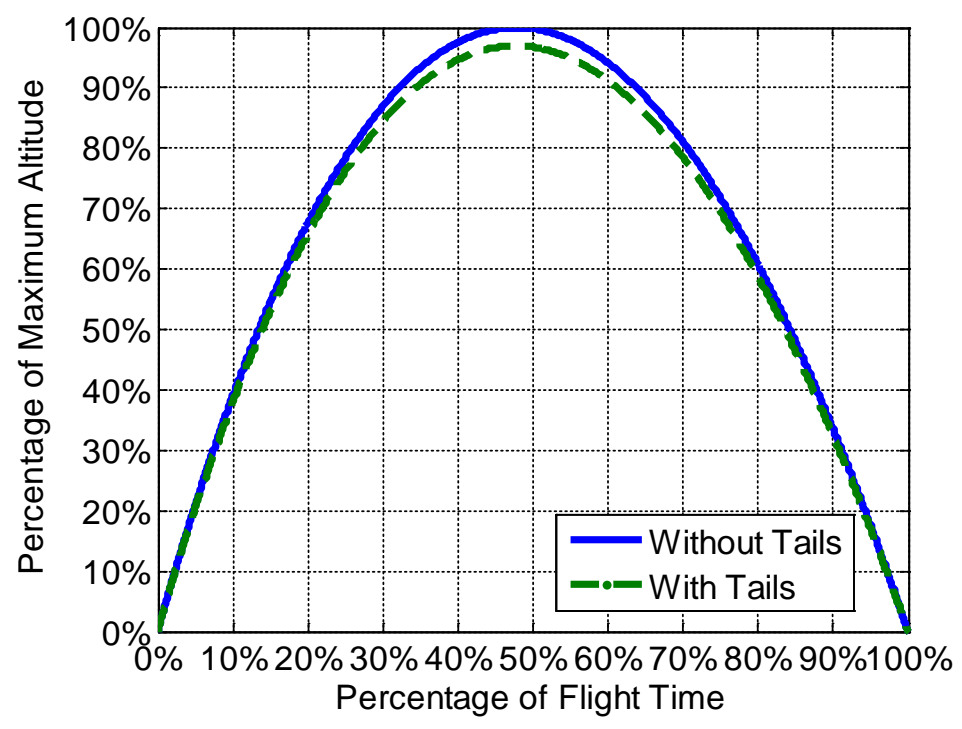

Figure 4-14: Z-directional Altitude Traveled 


\subsection{Heading Control}

After the HP was de-spun and roll leveled it was then desired to be guided to a particular heading or a specific offset distance from the line-of-fire. Feedback PID control was used as the control method for vertical tail deflections to control heading. Tuning of the PID controllers was done using the Simulink Control Design tuning tool.

This tool is found by selecting the "tune" button after the PID controller was opened and the Function Block Parameters was selected. Tuning was done by adjusting the response time and/or transient behavior sliders in Figure 4-15, and selecting a slider location that yielded the desired tuned response. This could not be used for tuning the roll controller because Matlab was not able to linearize the nonlinear equations associated with roll.

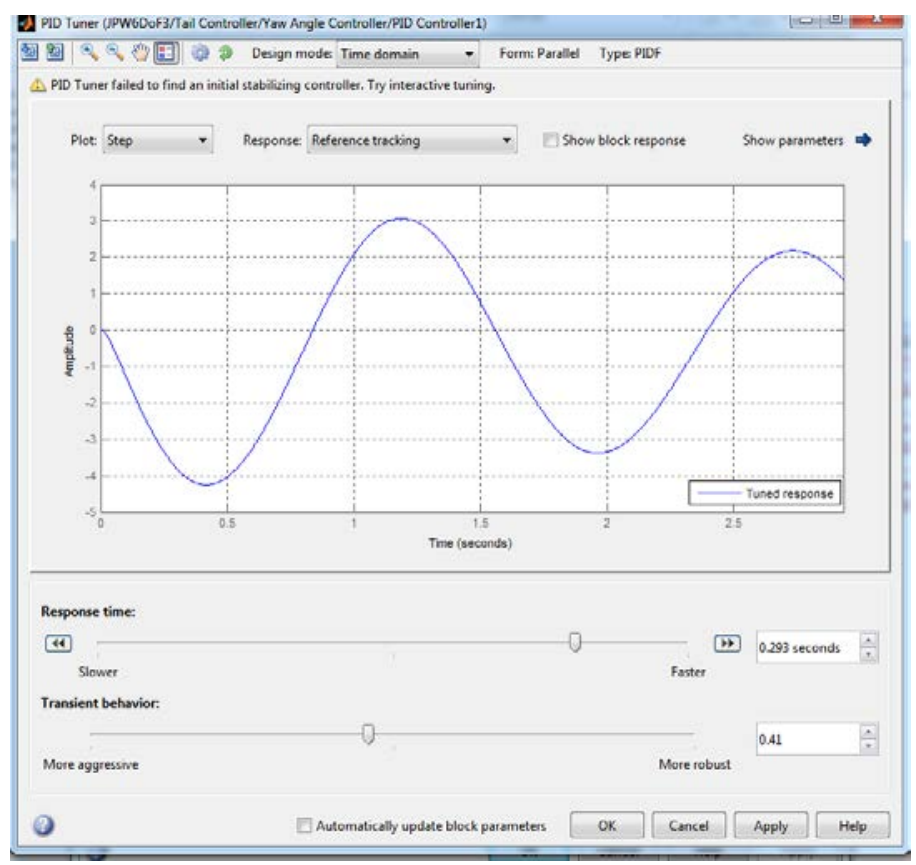

Figure 4-15: PID Tuner

The Heading Controller was set up to have two separate choices on how to input the desired heading. In Figure 4-16 the desired heading angle was an input in which the HP would track to that desired angle. However, in Figure 4-17, the heading control model allows the actual target location off the line of fire to be chosen. The inputs were desired distance and desired $\mathrm{Y}$ location, where the desire distance was the downrange distance from launch position and desired y location was the offset from the line of fire. In this loop, the heading angle required was determined from the difference of actual distance to required distance and difference in actual $\mathrm{Y}$ offset to the desired $\mathrm{Y}$ location. The inner loop of the heading controller drove the yaw rate error to zero, while the outer loop controller drove the heading error 
to zero. The heading controller was shown in Figure 4-18 in standard feedback form with all connections labeled.

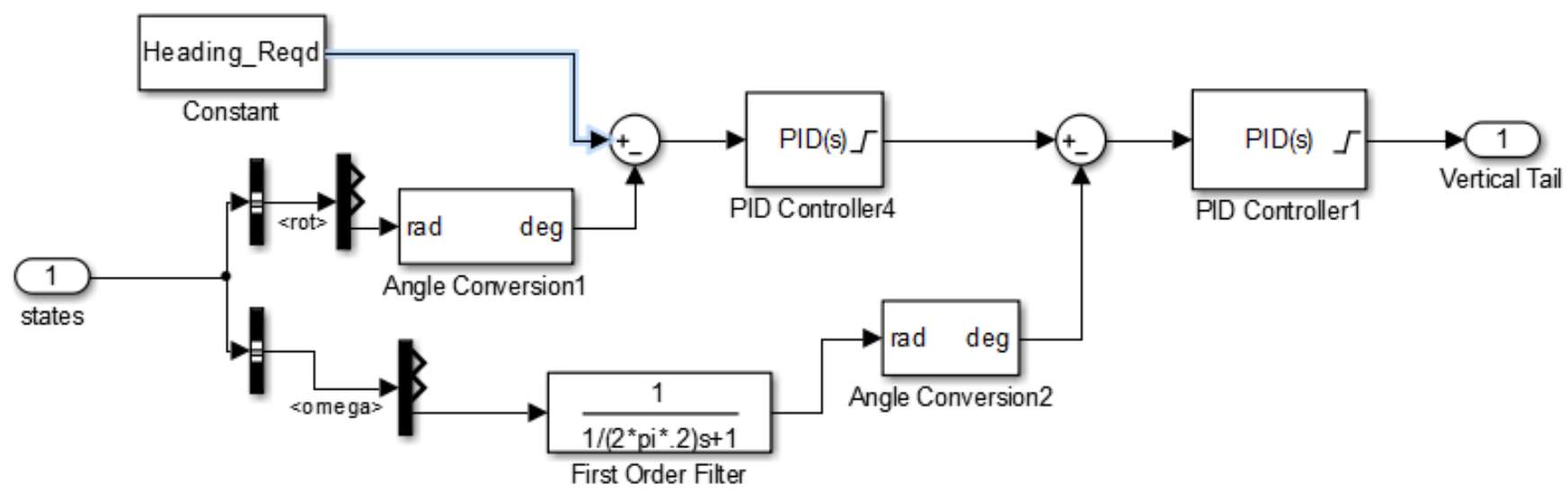

Figure 4-16: Heading Angle Tracking Sub-model

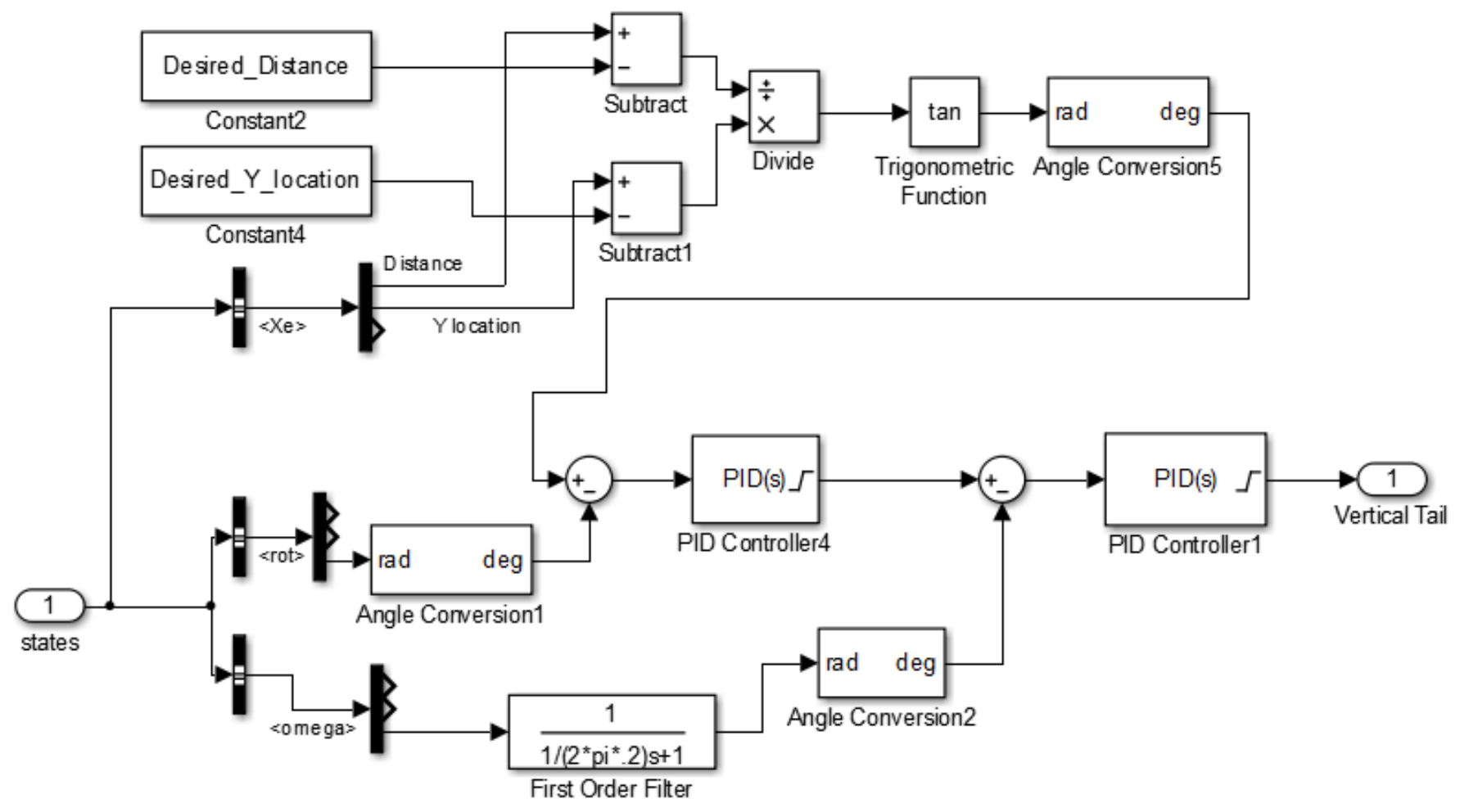

Figure 4-17: Desired Location Heading Tracking Sub-model 


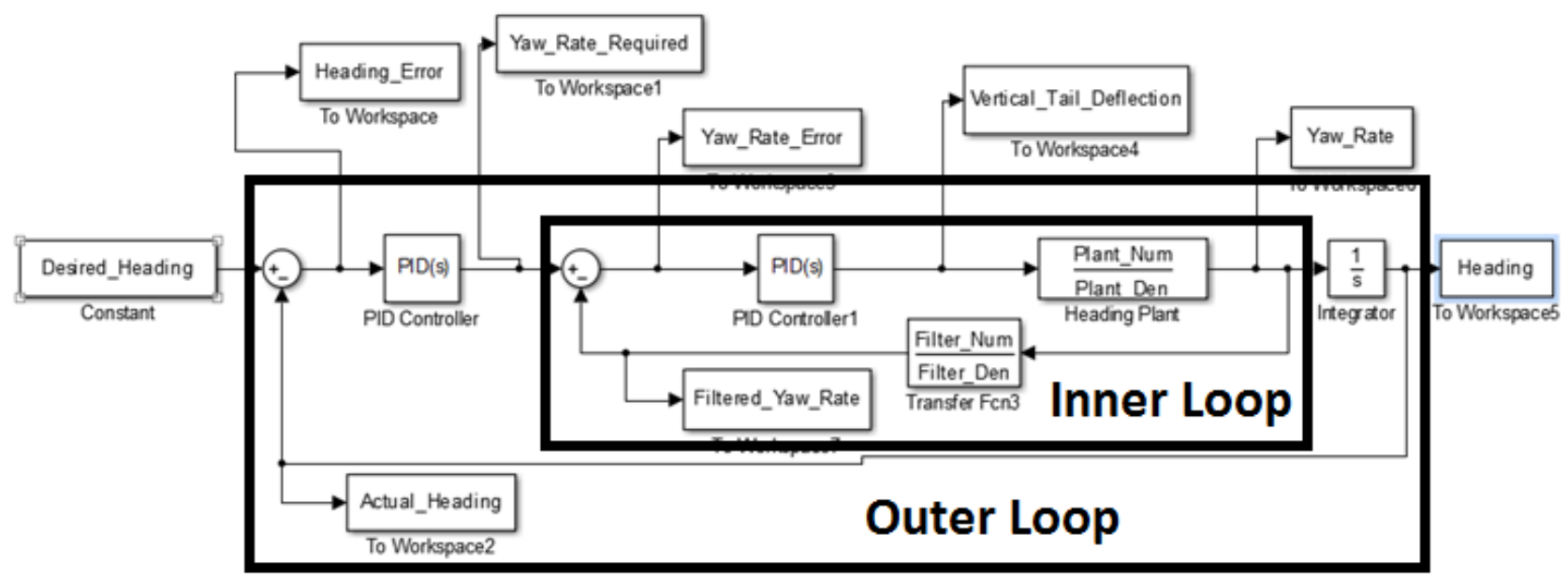

Figure 4-18: Inner and Outer Loops of Heading Controller

\subsubsection{Filter for yaw rate}

The yaw rate response in the simulation required a filter to remove the large magnitude oscillations created by a maneuver. This was required because the yaw rate controller was not able to drive error to zero since a tail maneuver would cause yaw rate oscillations many orders of magnitude higher than the steady yaw rate value from that tail deflection. A first order low pass filter was tested to see if it would damp the oscillations and yield the required response. In a first order low pass filter the cut off frequency $\left(\omega_{c}\right)$ could be manipulated to change the transfer function of the filter. The transfer function from a filter was shown as equation (4-3).

$$
\text { Filter } T F=\frac{1}{\frac{1}{2 \pi \omega_{c}} s+1}
$$

Multiple cut off frequencies were simulated to see which response most closely resembled the actual yaw rate response, without the large oscillations. Figure 4-19 shows the original yaw rate response and three low pass filters which were used to determine the cut off frequency that should be used. In Figure 4-19 the cut off frequency was varied from $0.15 \mathrm{~Hz}$ to $0.25 \mathrm{~Hz}$. 


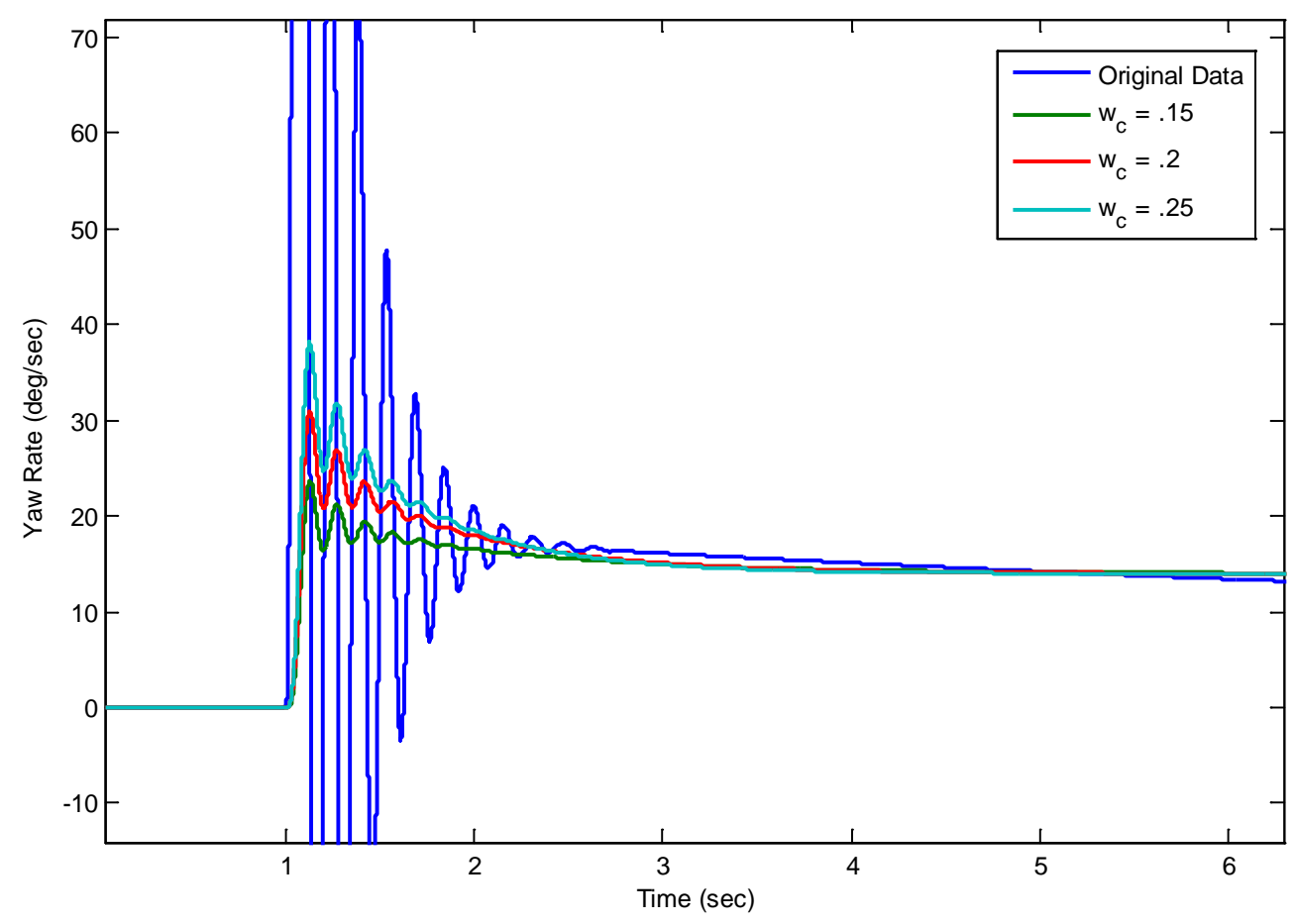

Figure 4-19: Yaw Rate for Multiple First Order Filters

Table 4-3: Yaw Rate Response Characteristics from Multiple Cut-off Filters

\begin{tabular}{|l|c|c|c|}
\hline \multicolumn{1}{|c|}{ Data Name } & Rise Time & Peak Time & Overshoot \% \\
\hline Original Data & $0.0069 \mathrm{sec}$ & $1.08 \mathrm{sec}$ & $3469 \%$ \\
\hline$\omega_{\mathrm{c}}=.15$ & $0.041 \mathrm{sec}$ & $1.13 \mathrm{sec}$ & $69 \%$ \\
\hline$\omega_{\mathrm{c}}=.2$ & $0.035 \mathrm{sec}$ & $1.13 \mathrm{sec}$ & $121 \%$ \\
\hline$\omega_{\mathrm{c}}=.25$ & $0.032 \mathrm{sec}$ & $1.13 \mathrm{sec}$ & $172 \%$ \\
\hline
\end{tabular}

A cut off frequency of $0.2 \mathrm{~Hz}$ was used in a first order cut off filter to damp the oscillations of yaw rate from vertical tail deflections. $0.2 \mathrm{~Hz}$ was chosen as the cut off frequency because it most closely resembles the response of the original yaw rate response from Table 4-3.

\subsubsection{Tuning Heading Controller (Inner and Outer Loop)}

The inner loop of the heading controller is the yaw rate controller that controls the vertical tail deflection to drive the yaw rate error to zero degrees per second. To set up the controller, the P, I, D and $\mathrm{N}$ values were set as a variable so that after tuning was complete the gain values could be saved, Figure 4-20. The tail deflections also have a maximum deflection of 10 degrees in the positive and negative 
direction. The tail deflection maximum deflection was set as the saturation limit in the PID Advanced tab of the PID controller, Figure 4-21.

Tuning and simulation of the PID gain values was done using a zero degree, flat, launch angle with the gravitation vector as zero magnitude. The other initial conditions described in Table 2-1 were used as well. This was done so the projectile would not be on a trajectory, but on a level path that it could be turned from. This assumption could be made since the projectile was at a zero roll angle and the gravitational force was only in the z-direction.

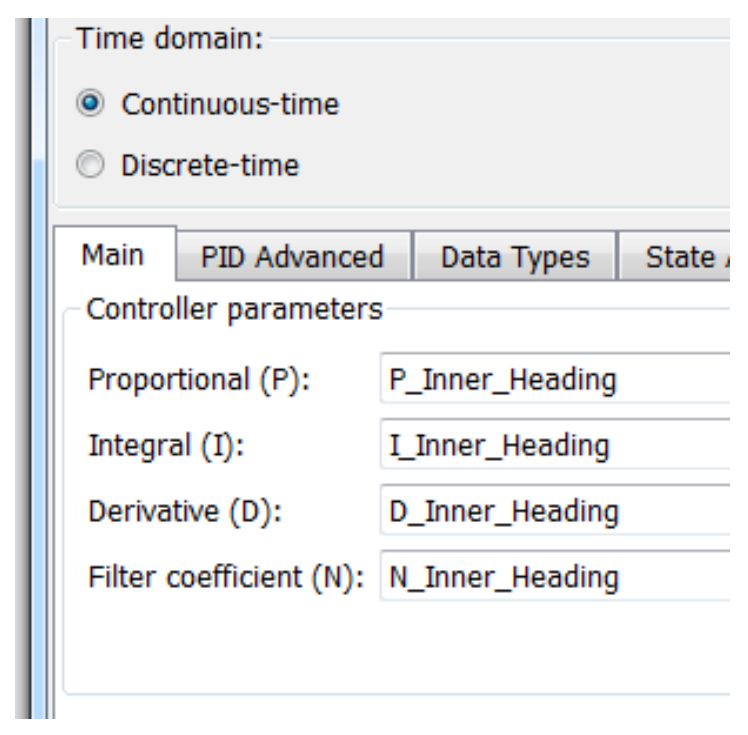

Figure 4-20: Setting Variables for Gain Values

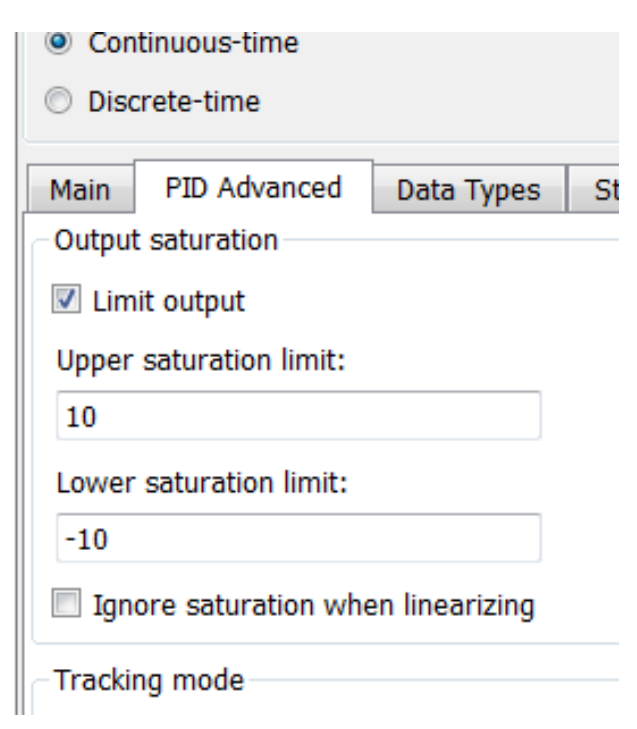

Figure 4-21: Setting Saturation Limit

The yaw rate desired was the input to the right summing junction, as shown in Figure 4-22, to tune the inner loop PID controller. In this case the yaw rate desired was chosen to be 5 degrees per second. A value of 5 degrees per second was chosen because it was a value achievable by tail deflections within \pm 10 degrees.

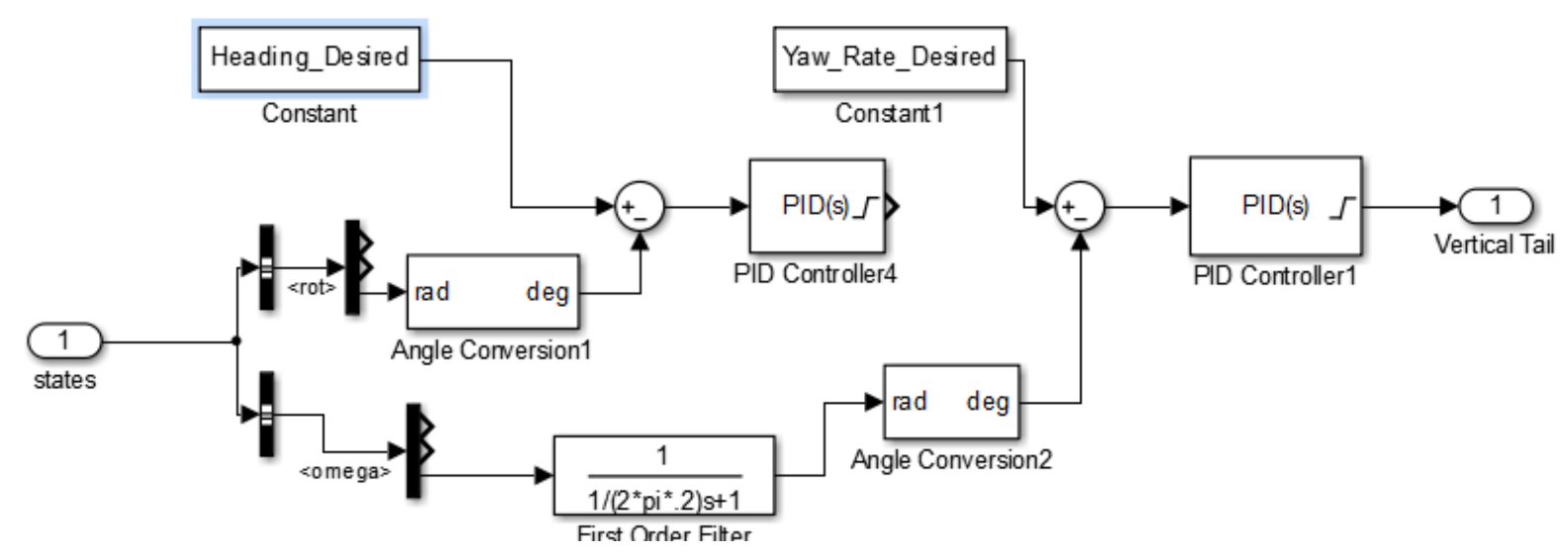

Figure 4-22: Heading Controller with Inputs for Tuning 
Using the Tune function of the PID controller the yaw rate controller was tuned to have the smooth first order response by sliding the response time slider to 1.93 seconds, Figure 4-23. In Figure 4-23 the tuned response was based on a unit step function.

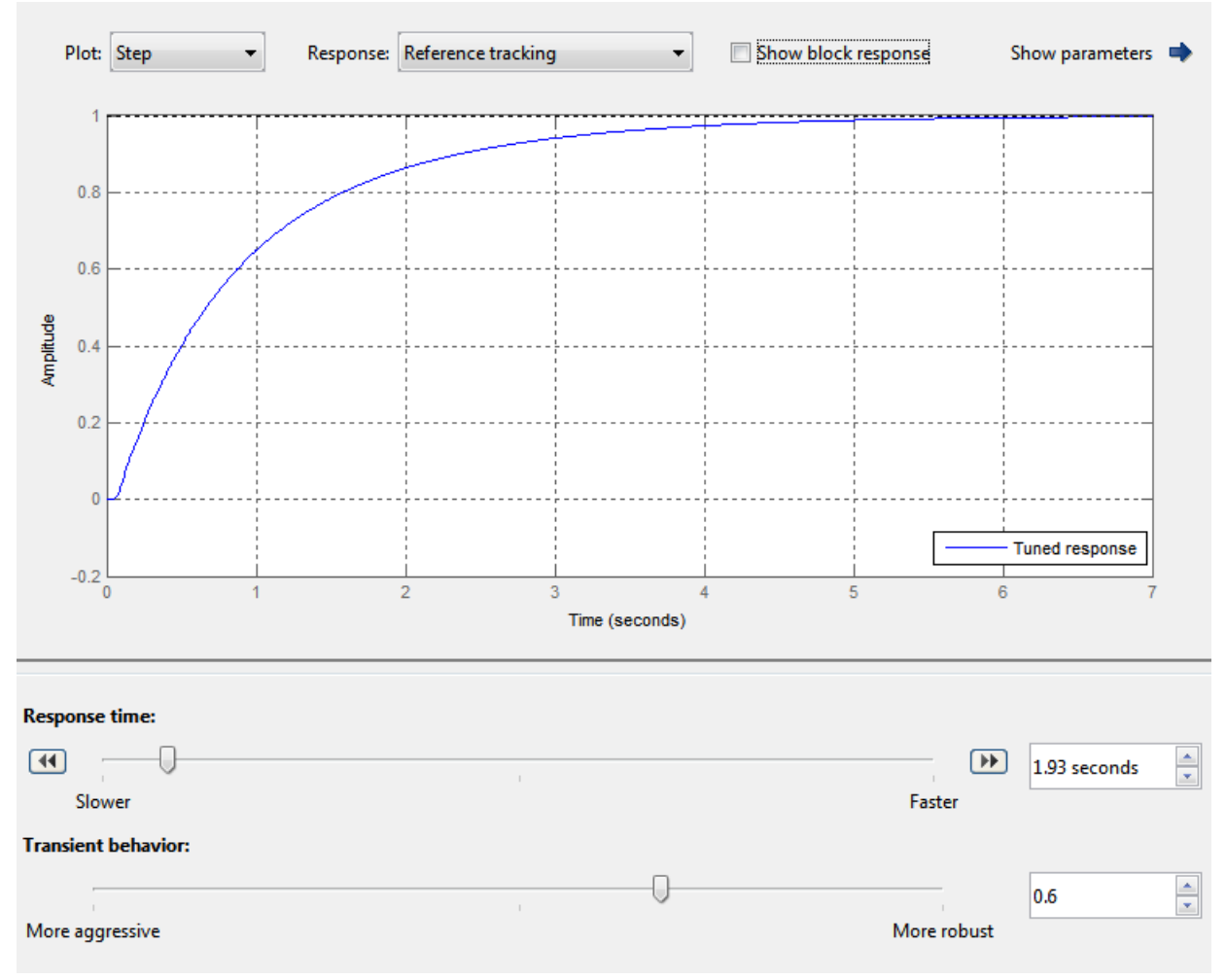

Figure 4-23: Initial Yaw Rate Controller Tuning

After the PID gain values were determined by manipulating the slider bars, the projectile was simulated to see the vertical tail deflection required and if the yaw rate error goes to zero. From Figure 4-24, the PID gain values from the response of Figure 4-23 resulted in the yaw rate error going to zero while the vertical tail deflections did not oscillate and were within the \pm 10 degree limit. The PID gain values in Table 4-4 were used moving forward to the outer loop of the heading controller. 
Table 4-4: PID Values from Yaw Rate Tune

\begin{tabular}{|c|c|}
\hline P_Inner_Heading & 0 \\
\hline I_Inner_Heading & -.443 \\
\hline D_Inner_Heading & 0 \\
\hline N_Inner_Heading & 100 \\
\hline
\end{tabular}
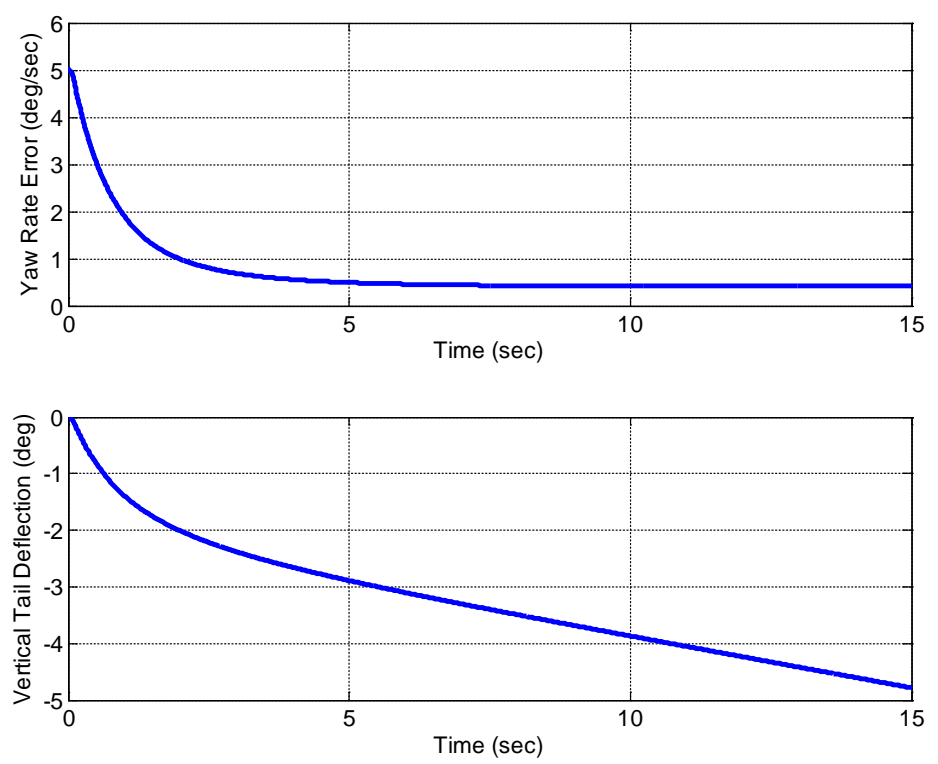

Figure 4-24: Yaw Rate Error and Vertical Tail Deflection Response from PID tune

The outer loop of the Heading controller was used to determine the yaw rate required to get to a particular heading angle. In this case heading angle error was the input into the PID controller and the output was the required yaw rate. In the PID controller block there was the ability to change the saturation limit, similar to the maximum tail deflection in the inner loop PID controller. The saturation limit was determined by simulating the maximum deflection of 10 degrees. The yaw rate response from the 10 degree tail deflection was used to determine the saturation limit. The response of yaw rate was evaluated in the Curve Fitting Tool of Matlab to determine that the y intercept was -23.33, Figure 4-25. The yintercept was used as the saturation limit because it was the maximum yaw rate achievable without oscillations. Meaning that \pm 23.33 was used as the saturation limit. 


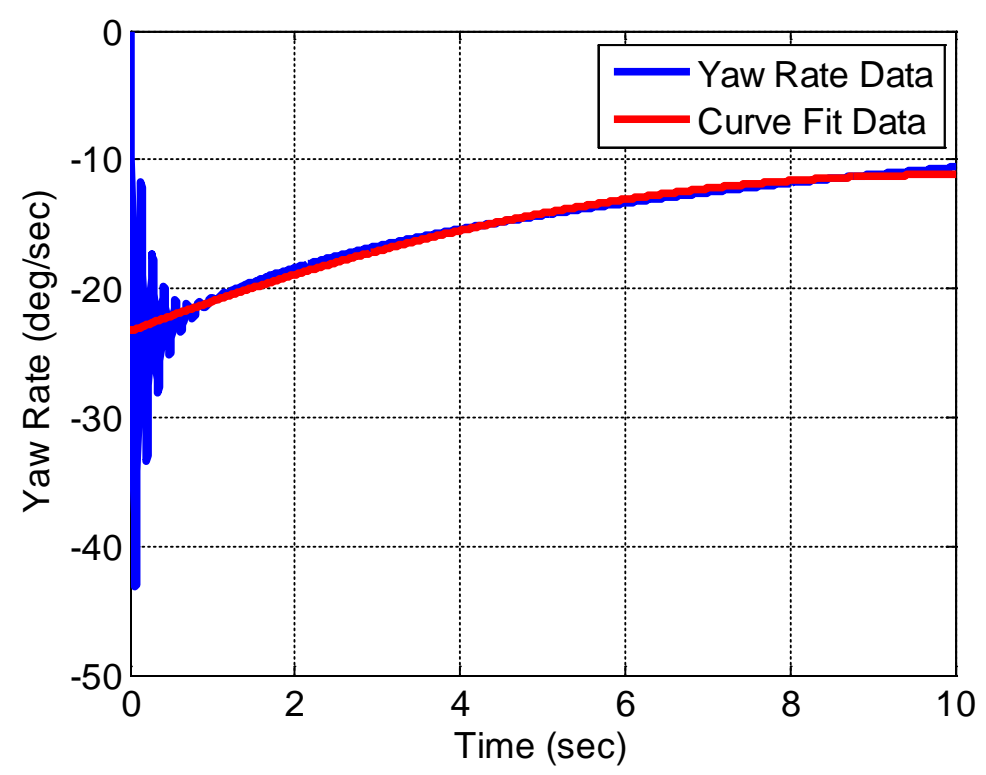

Figure 4-25: Yaw Rate Response from 10 deg. Vertical Tail Deflection

By choosing a desired heading and connecting the outer loop PID controller with the summation junction at yaw rate desired the PID slider can be tuned similarly to the inner loop. In Figure 4-26, the response time slider was chosen as 0.303 seconds because the response to a unit step input was a smooth response with minimal overshoot and approximately 2.5 second settling time.

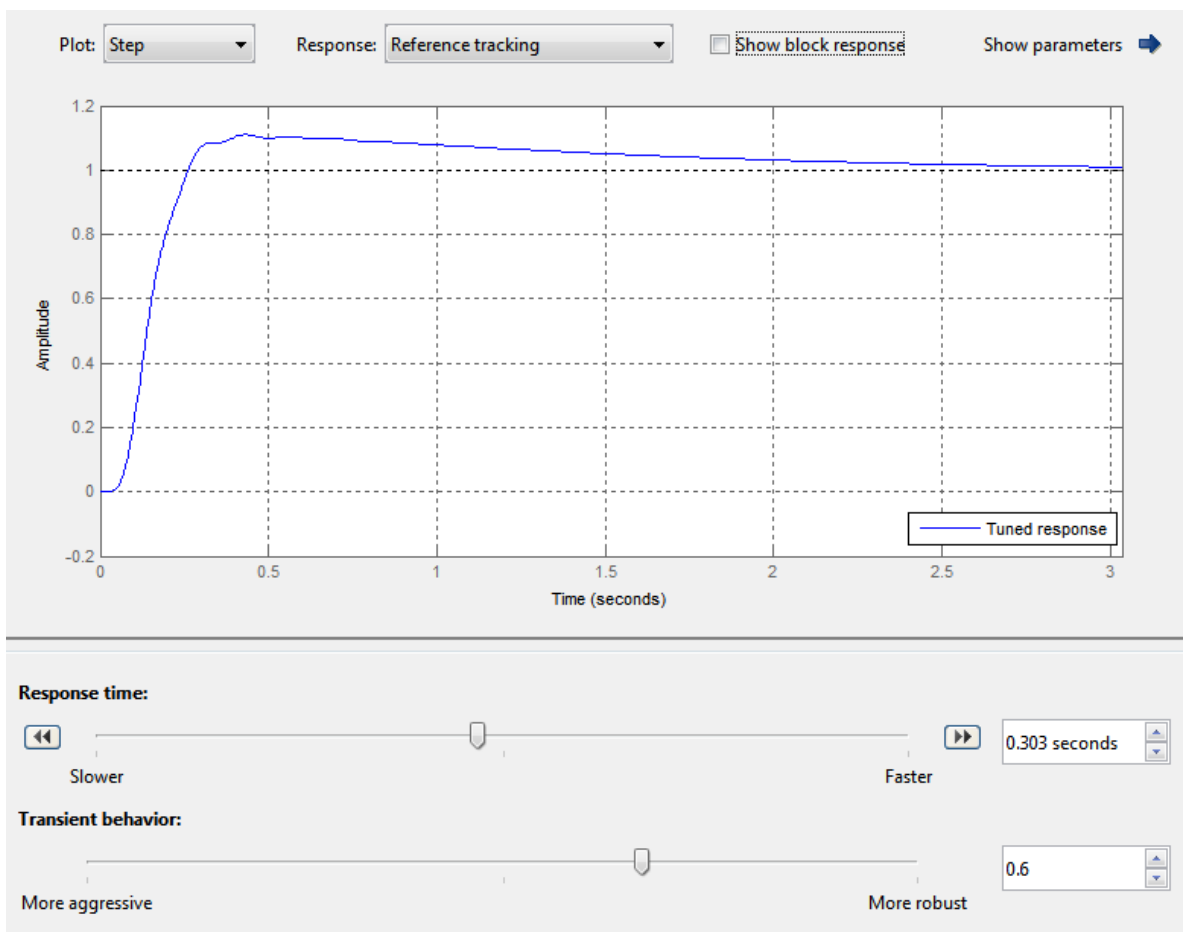

Figure 4-26: Initial Heading Angle Control Tune 
The response from the heading PID tune in Figure 4-26 was simulated to determine if the heading error went to zero degrees. In Figure 4-27 the heading error went to zero within approximately four seconds. The PID gain values from the tune of this controller were compiled in Table 4-5.

Table 4-5: PID Values from Heading Tune

\begin{tabular}{|c|c|}
\hline P_Outer_Heading & 6.7758 \\
\hline I_Outer_Heading & 0.8470 \\
\hline D_Outer_Heading & 0 \\
\hline N_Outer_Heading & 100 \\
\hline
\end{tabular}
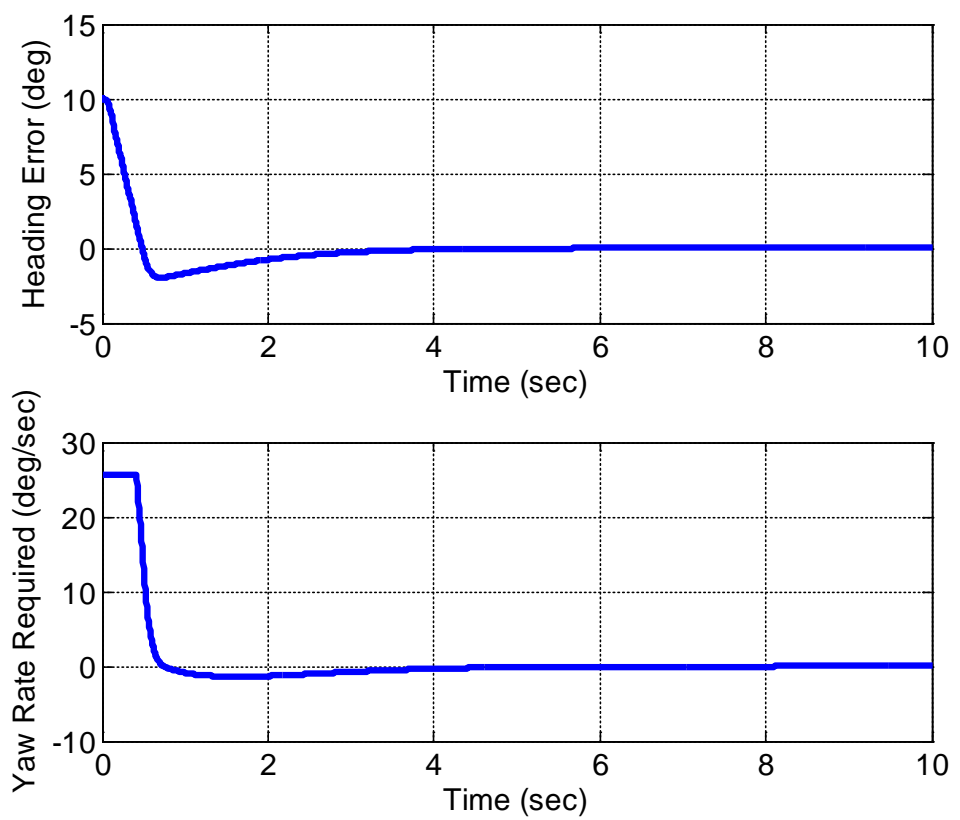

Figure 4-27: Heading Error and Yaw Rate Required from PID Tune

When investigating the steady state error by viewing the heading value at 10 seconds it was noticed that the steady state error was $4 \times 10^{-4}$ degrees. This was not a significant value but was changed easily by manually adjusting the integral gain value "I_Outer_Heading”. The outer loop integral value was adjusted to zero and not only was the steady state error removed but the settling time was reduced, as seen from Figure 4-27 to Figure 4-28. The final PID values from both controllers that were used moving forward were shown in Table 4-6. 
Table 4-6: Final Heading Control PID Values

\begin{tabular}{|c|c|}
\hline P_Inner_Heading & 0 \\
\hline I_Inner_Heading & -.443 \\
\hline D_Inner_Heading & 0 \\
\hline N_Inner_Heading & 100 \\
\hline P_Outer_Heading & 6.7758 \\
\hline I_Outer_Heading & 0 \\
\hline D_Outer_Heading & 0 \\
\hline N_Outer_Heading & 100 \\
\hline
\end{tabular}

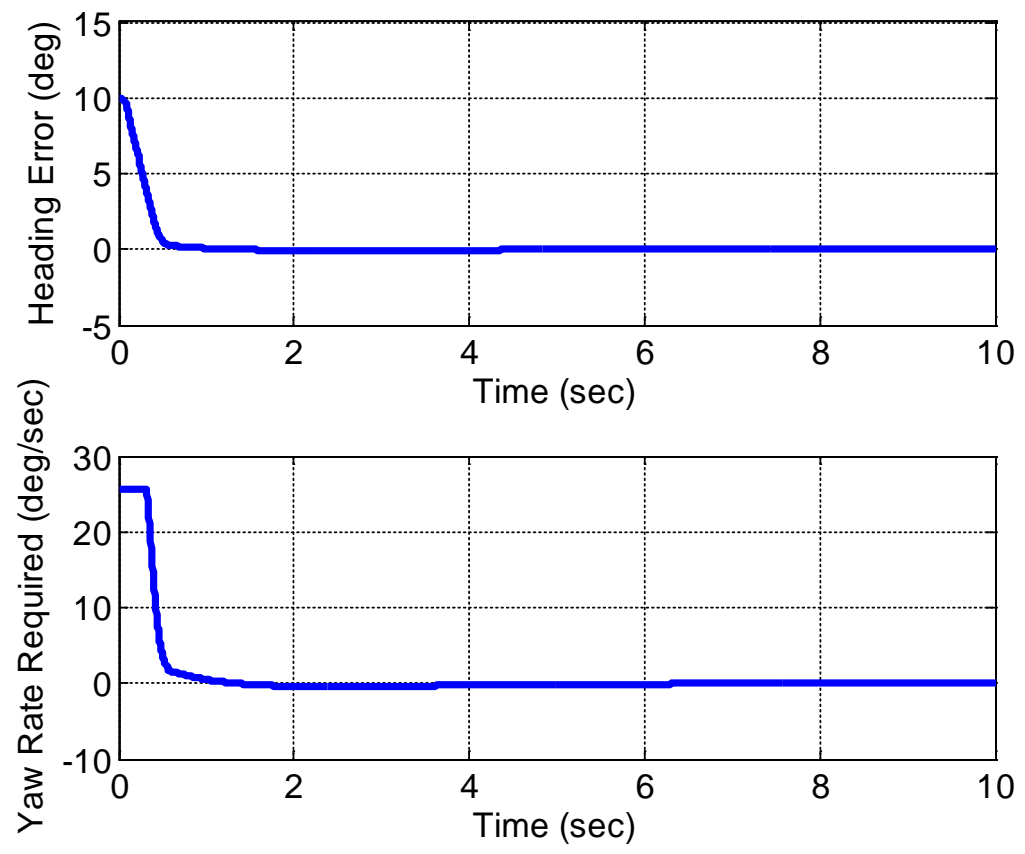

Figure 4-28: Final Outer Loop PID Tune

\subsubsection{Heading Controller Testing}

Using the PID values compiled in Table 4-6, the heading tracking was tested by simulating the projectile at the conditions described in Table 2-1. However, in this simulation the initial roll rate was zero degrees per second, since the heading control was not used until the HP was roll-leveled. In the first test, multiple heading angles were evaluated to see if the projectile could track to different heading angles. From Figure 4-29 the projectile was simulated to track to 10, 30, 50 and 70 degrees. For each required heading simulation the projectile was able to track to the required heading angle. Figure 4-29 also shows the vertical tail position required for each simulation. 

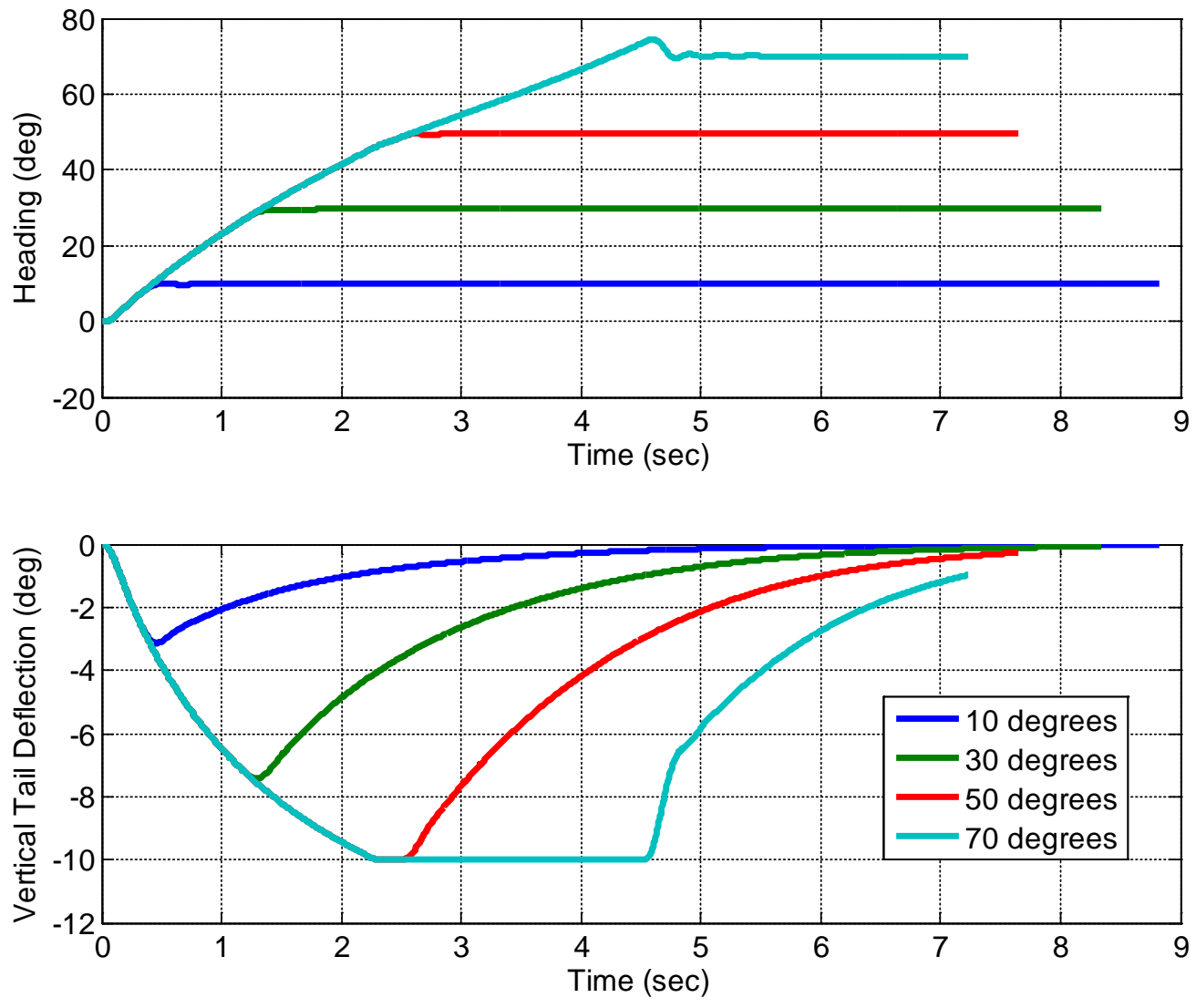

Figure 4-29: Simulation Test of Multiple Heading Angles

In Figure 4-17 there was also a method of tracking the projectile to a specific Y-offset from the line of fire and downrange distance. Guidance to a desired offset from the line of fire at a specific downrange distance was tested by simulating two separate impact point cases. In these simulations the HP's altitude was not controlled yet, so the projectile crossed through the desired location but may not land there. Figure 4-30 shows the simulation results for the Y-offset controller of the target locations of Table 4-7. From these simulations it was seen that the Y-offset controller could be used to accurately guide a simulated projectile to a desired Y-offset location at a specified distance. Distance from projectile to target, as it passes through the desired distance, were also shown as the offset in Table 4-7.

Table 4-7: Desired Target Locations for Y-Offset Control

\begin{tabular}{|l|c|c|}
\hline & Simulation 1 & Simulation 2 \\
\hline Desired Downrange Distance & $\begin{array}{c}75 \% \text { of Max. } \\
\text { Unguided Range }\end{array}$ & $\begin{array}{c}87.5 \% \text { of Max. } \\
\text { Unguided Range }\end{array}$ \\
\hline Desired Y-Offset Distance & 1250 Projectile & 2500 Projectile \\
\hline
\end{tabular}




\begin{tabular}{|l|c|c|}
\hline & Diameters Right & Diameters Left \\
\hline Offset from Desired Target & 0.7 meters & 1.4 meters \\
\hline
\end{tabular}

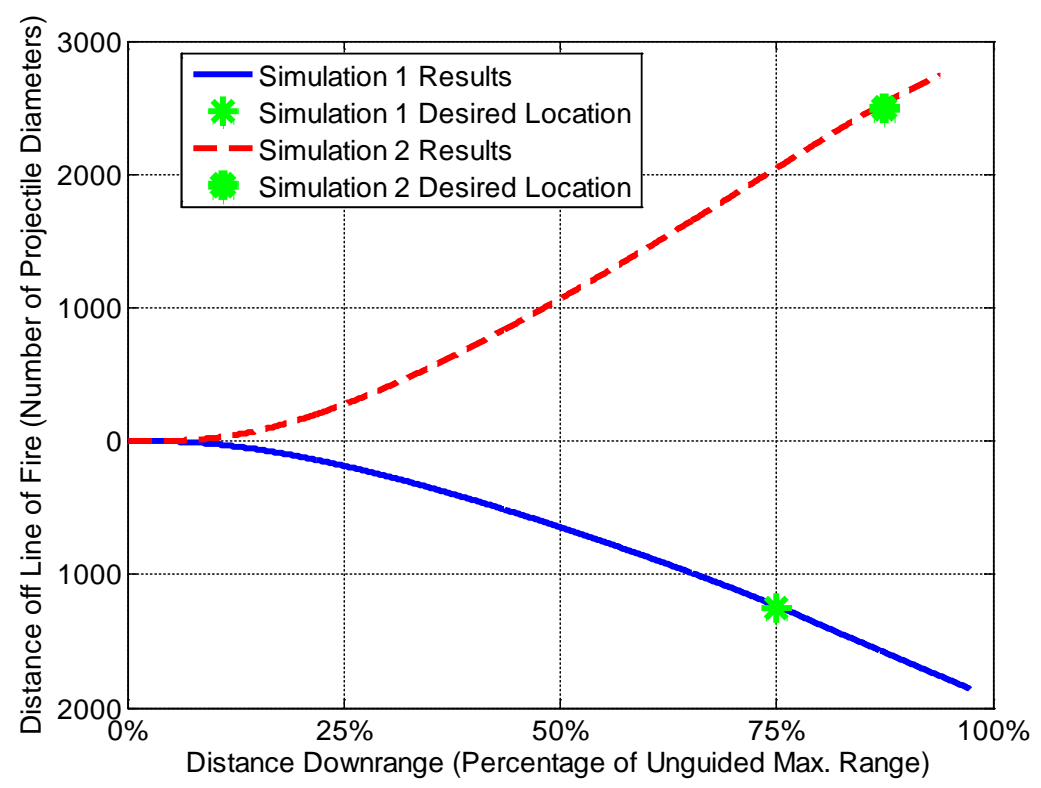

Figure 4-30: Desired Y Offset Simulation Tests

\subsection{Longitudinal Control}

The longitudinal controller sub-model of the tail controller model in Figure 4-2 was shown as Figure 4-31. The longitudinal controller used the states as an input for the HP's current position, velocity and pitch angle. This controller was used to determine the horizontal tail deflections required for the HP to be on its maximum range trajectory, and then used its current states to estimate its ballistic trajectory Its tails were then deflected back to zero when the estimated trajectory reached the desired impact point. 


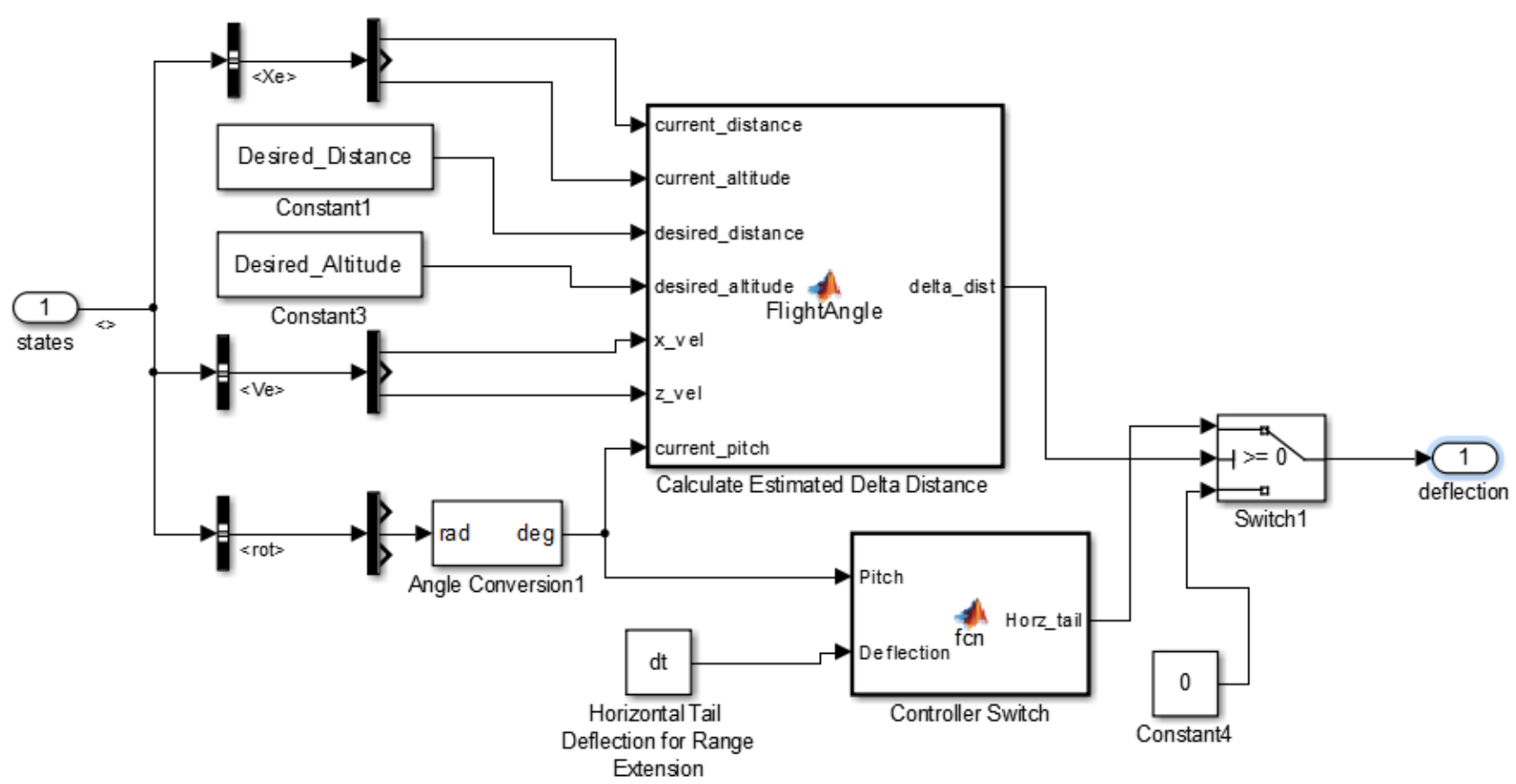

Figure 4-31: Distance/Altitude Longitudinal Controller Sub-system

\subsubsection{Method}

The longitudinal controller consisted of a three step process to guide the HP to a desired downrange distance and altitude. The three steps were:

1. Deflected the horizontal tails so the HP was on its maximum range trajectory.

2. Estimated the ballistic trajectory of the HP with the tails deflected back to zero degrees.

3. Deflected the tails to zero degrees when its estimated trajectory crossed the desired location.

Step one was achieved by determining the tail deflection magnitude, pitch angle for tail deflection and launch angle of the HP for maximum range. Then while the HP was on its maximum range trajectory kinematic equations were used to estimate the future trajectory of the HP if the tails were deflected back to zero degrees. When this estimated trajectory crosses the desired point of impact the controller was then used to deflect the tails back to zero degrees.

\subsubsection{Find deflection magnitude, launch angle and pitch for tail deflection for max range}

The tail deflection which resulted in the maximum range extension was determined by simulating multiple tail deflection magnitudes. The tails were deflected at the top of the trajectory. The launch angle was initially set to 45 degrees because launch angle for maximum range was not determined yet. From Figure 4-32 a horizontal tail deflection of 10 degrees resulted in maximum range extension for the HP with constants from Table 2-1. 

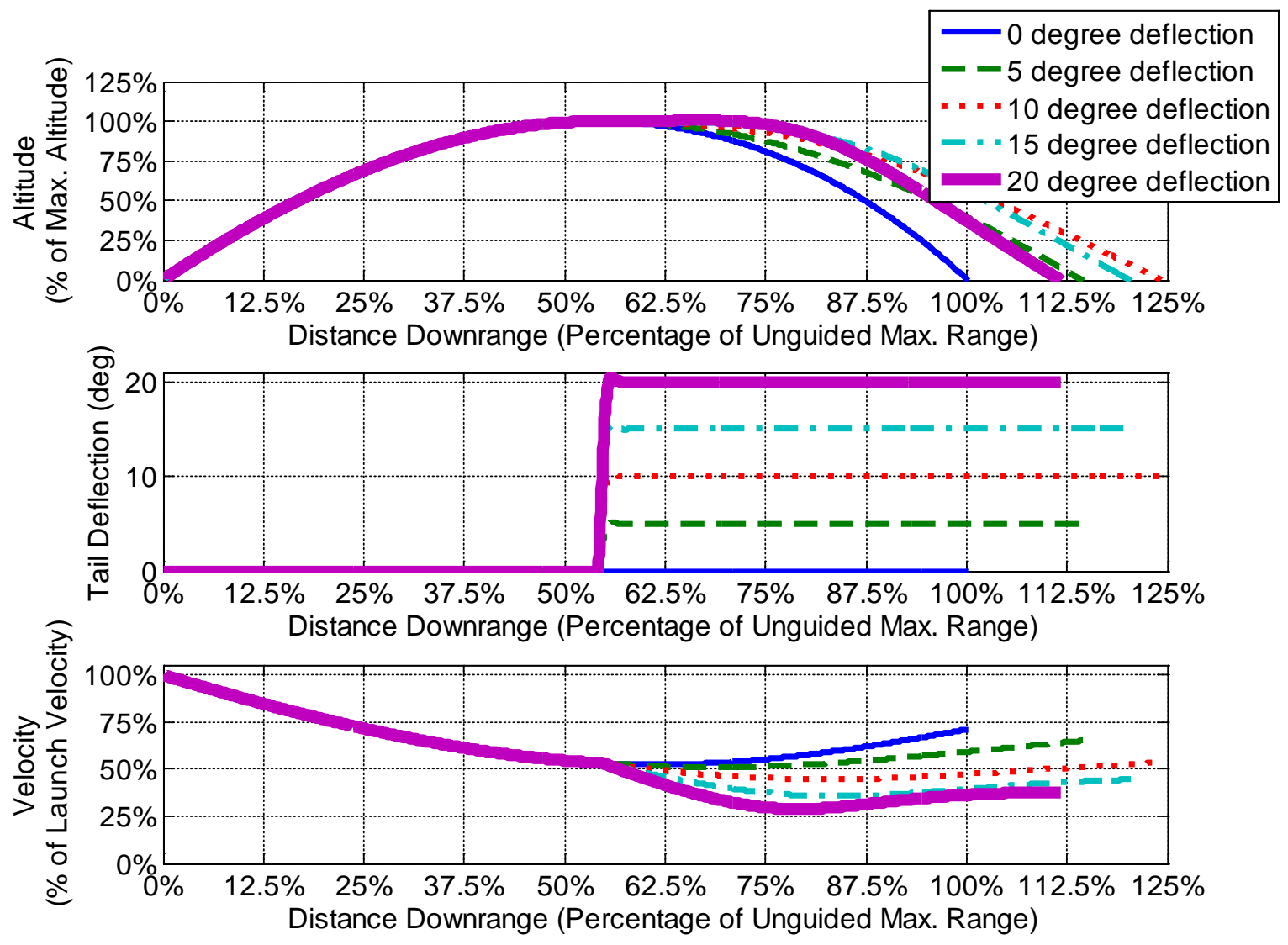

Figure 4-32: Horizontal Tail Deflection for Maximum Range

With the tail deflection for maximum range now determined, the pitch angle of the HP, when the tails were deflected, that yielded the maximum range extension was determined. The pitch was used to determine when the tails were deflected for maximum range so that the munition would not need to be reprogrammed for launch, as investigated in (Gioia 2013). The pitch angle was varied from 10 degrees to negative 10 degrees and it was determined that the maximum range occurs when the tails were deflected when the projectile was at negative 3.3 degrees pitch, from Figure 4-33 and Figure 4-34. 


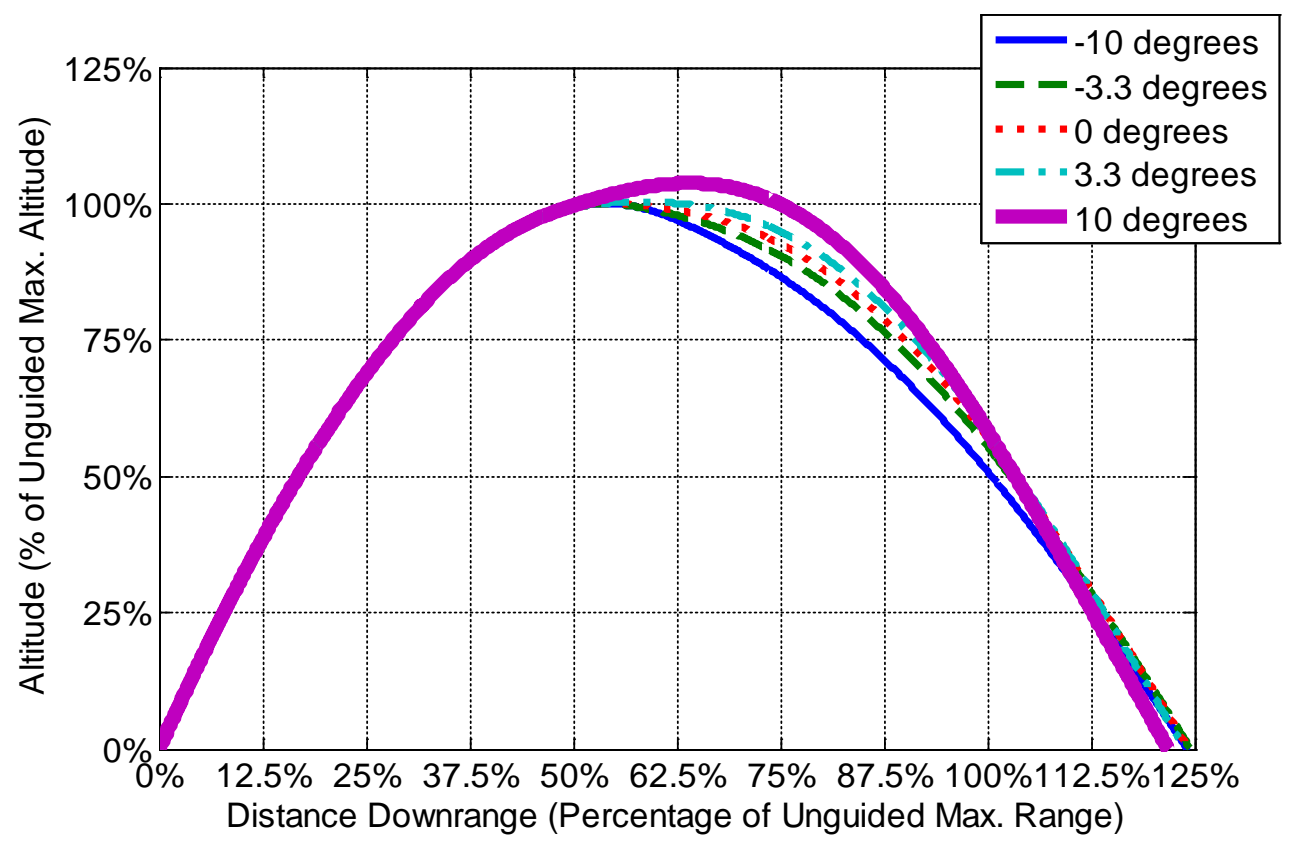

Figure 4-33: Find Maximum Range with Change in Deflection Pitch

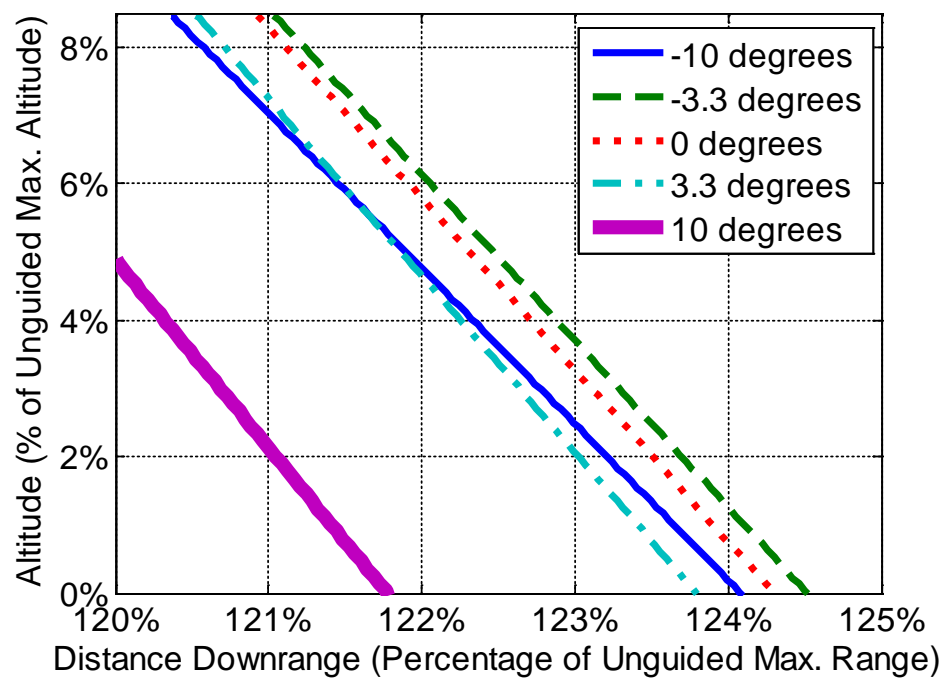

Figure 4-34: Find Maximum Range with Change in Deflection Pitch (Zoomed to Max Range)

It was now important to update the initial launch angle with the launch angle for maximum range.

In Figure 4-35, the maximum range occurred from a 39.5 degree launch angle. A launch angle of 45 degrees does not allow for the maximum distance of this projectile because of drag. The projectile must efficiently convert kinetic energy to potential energy than back to kinetic energy. With drag, the most efficient situation was when the projectile was launched at 39.5 degrees. A 39.5 degree launch angle yielded a $25.8 \%$ range extension over the non-guided projectile which was greater than $24.5 \%$, the range extension from a 45 degree launch angle. 


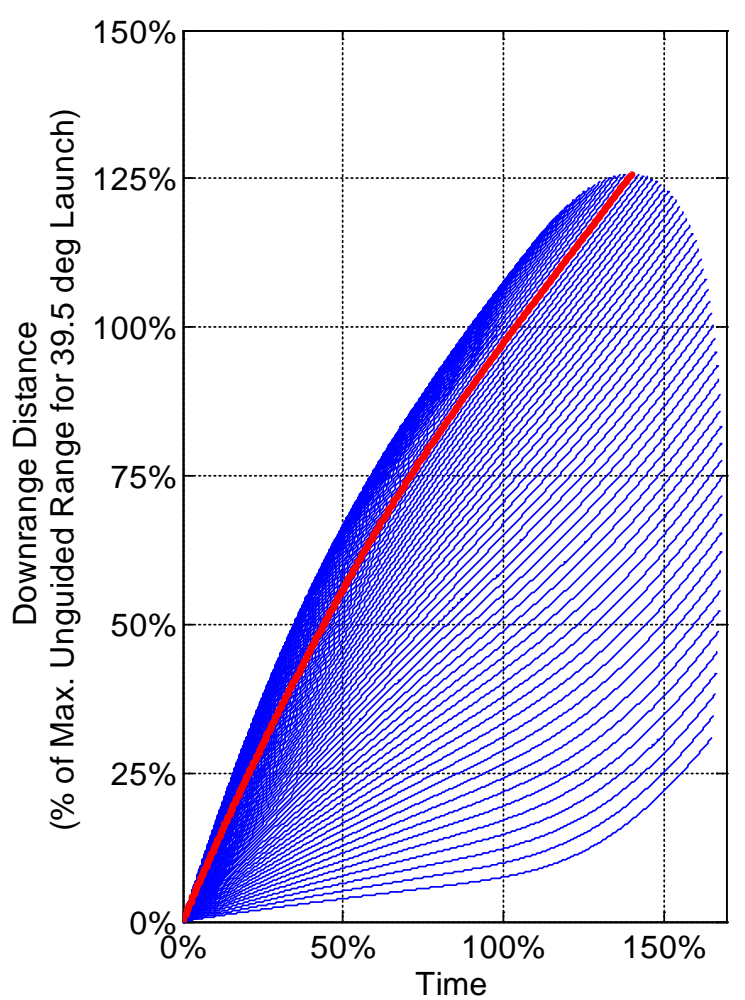

(\% of Unguided Flight Time for 39.5 deg Launch)

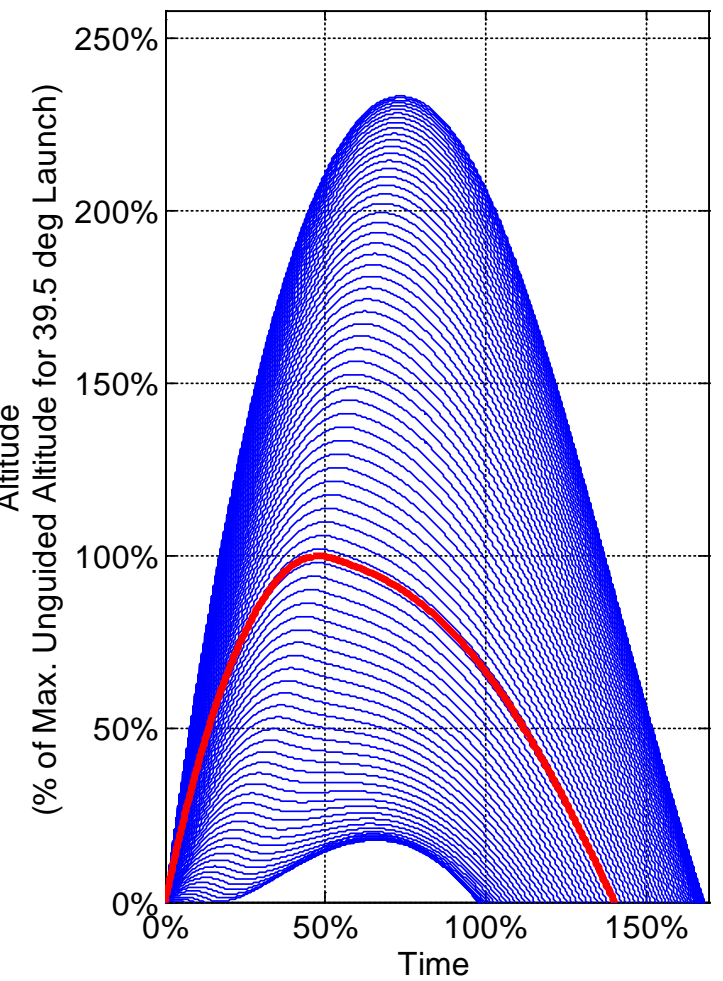

(\% of Unguided Flight Time for 39.5 deg Launch)

Figure 4-35: Distance and Altitude of 1 to 87 degree launch angle

\subsubsection{Range Extension and Guidance}

The ‘Calculate Estimated Delta Distance’ sub-system from Figure 4-31 was created to determine the difference between the required distance and the estimated distance calculated for the accelerations caused by the tails being deflected back to zero degrees. The estimated distance was determined by completing a force balance acting on the projectile, with the free-body diagram in Figure 4-36 then solving the equations of motion. The sub-system contained a while loop that determined when the projectile crossed the desired altitude along its current ballistic trajectory. At that altitude the distance downrange was recorded. If the estimated distance from the while loop became greater than the desired distance the switch function in Figure 4-31was used to deflect the tails back to zero degrees.

The acceleration of the projectile due to lift and drag was determined for the $\mathrm{x}$ and $\mathrm{z}$ directions. The forces acting on the HP that effected the acceleration were lift, drag and gravitational force. The forces were used to determine the acceleration by dividing by the projectiles mass. In equations (4-3) and (4-4) the lift coefficient, drag coefficient and angle of attack produced was required. This data was collected through simulation results in Section 4.5.4. The free body diagram used to determine the accelerations was Figure 4-36 with the accelerations as equations (4-3) and (4-4). At each time step the pitch and angle of attack were summed together, as shown in Figure 4-36. The velocity and position in the 
$\mathrm{x}$ and $\mathrm{z}$ direction were determined by taking the first and second derivative of the acceleration in each direction, respectively. The velocity and position were using solved using a discrete time step $(\Delta t)$. The velocities were determined by adding the velocity at the previous time step to the acceleration multiplied by the time step in equations (4-5) and (4-6). The pitch had to be recalculated at each time step by using equation (4-7), due to the change in velocity vector and angle of attack. Similar to calculating the velocity, the position of the HP was calculated using equations (4-8) and (4-9).

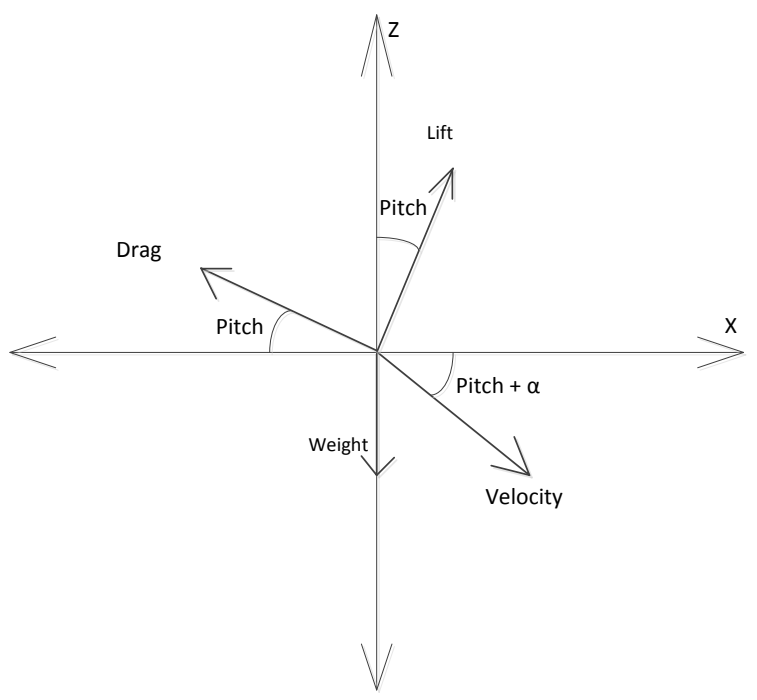

Figure 4-36: FBD used to determine accelerations on projectile

$$
\begin{gathered}
a_{z}=-g+\cos (\mid \text { Pitch } \mid) C_{L} \frac{\cdot 5 \rho S V^{2}}{M_{p}}+\sin (\mid \text { Pitch } \mid) C_{D} \frac{.5 \rho S V^{2}}{M_{p}} \\
a_{x}=\sin (\mid \text { Pitch } \mid) C_{L} \frac{\cdot 5 \rho S V^{2}}{M_{p}}-\cos (\mid \text { Pitch } \mid) C_{D} \frac{.5 \rho S V^{2}}{M_{p}} \\
V_{z_{i}}=V_{z_{i-1}}+a_{z} \Delta t \\
V_{x_{i}}=V_{x_{i-1}}+a_{x} \Delta t \\
\text { Pitch }=\tan ^{-1}\left(\frac{V_{z_{i}}}{V_{x_{i}}}\right)+\alpha \\
z_{i}=z_{i-1}+V_{z_{i}} \Delta t \\
x_{i}=x_{i-1}+V_{x_{i}} \Delta t
\end{gathered}
$$

\subsection{4 $C_{L}, C_{D}$ and $A o A$ when tails are deflected to zero degrees}

The lift coefficient, drag coefficient and angle of attack could be determined due to the tails being deflected from ten to zero degrees using the simulator.ly to zero degrees. These values were estimated by saving each to be used at each time step after the tails start moving to zero deflection. The vector values were saved at a $200 \mathrm{~Hz}$ time step starting from the time where tails had a command to go to zero degrees 
from 10 degrees. Figure 4-37 shows the total lift coefficient, total drag coefficient and angle of attack after the tails were deflected to zero degrees at eight seconds.
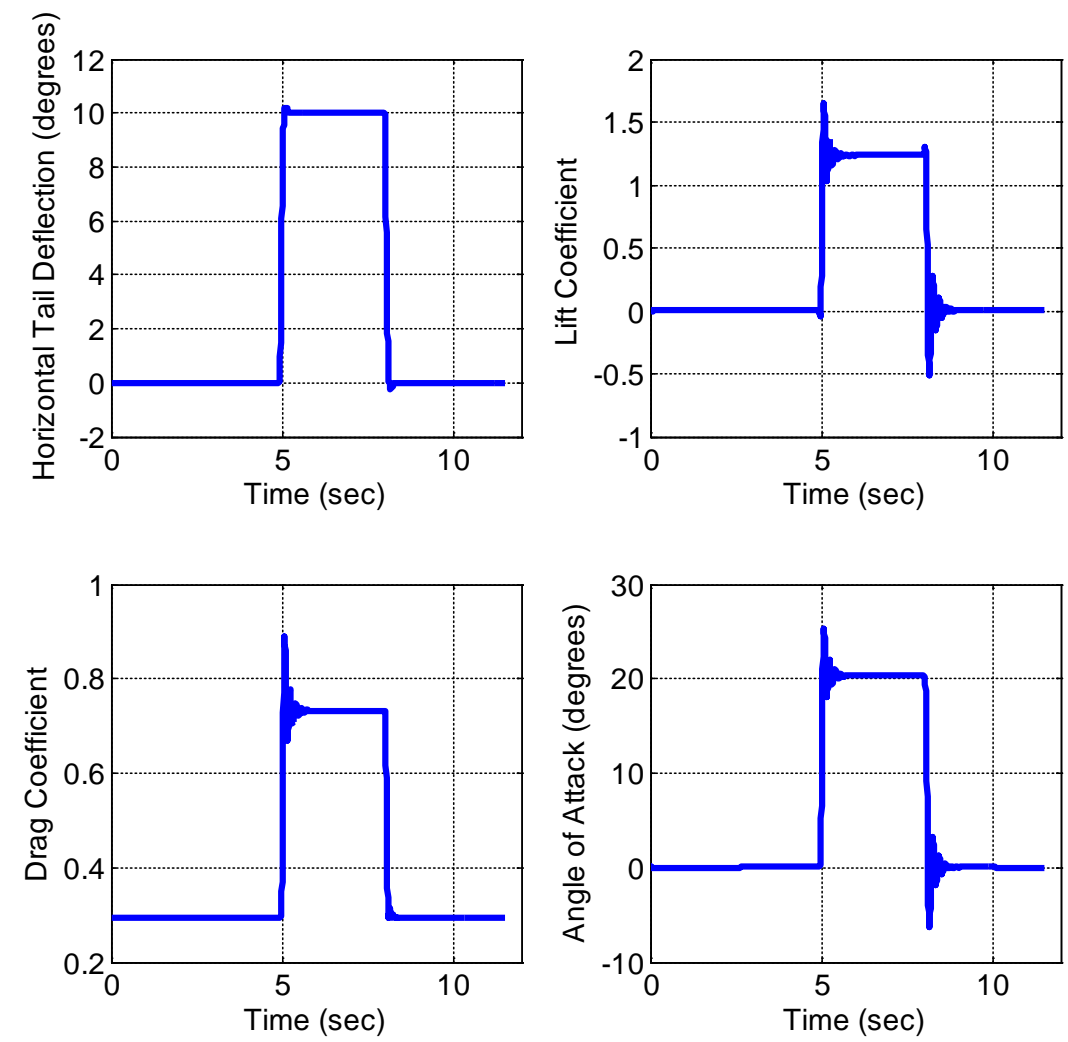

Figure 4-37: Create Vectors of CL CD and AoA from Tail Deflection

All the variables in equations (4-3) and (4-4) were now known and were used to estimate the ballistic trajectory of the HP. When the ballistic trajectory estimated passed through the desired point of impact (delta_dist=0) the tails were deflected back to zero degrees using the switch function.

\subsubsection{Longitudinal Controller Testing}

The inputs of two different on line-of-fire impact points were chosen to test the longitudinal controller. The simulated results and the desired results were compared. In Figure 4-38, the unguided trajectory was compared with the guided trajectory and the horizontal tail deflection for the targeting was shown as well. In Figure 4-38 the desired distance was $122.5 \%$ of the unguided projectiles maximum range at 0\% maximum unguided altitude. In Figure 4-39 the desired distance was 100\% of the unguided projectiles maximum range at 50\% maximum unguided altitude. The results of the simulations were shown in Table 4-8. These figures show that the longitudinal controller was able to achieve the task of guiding the projectile to a desired distance and altitude within 22.5 projectile diameters of the desired location. 

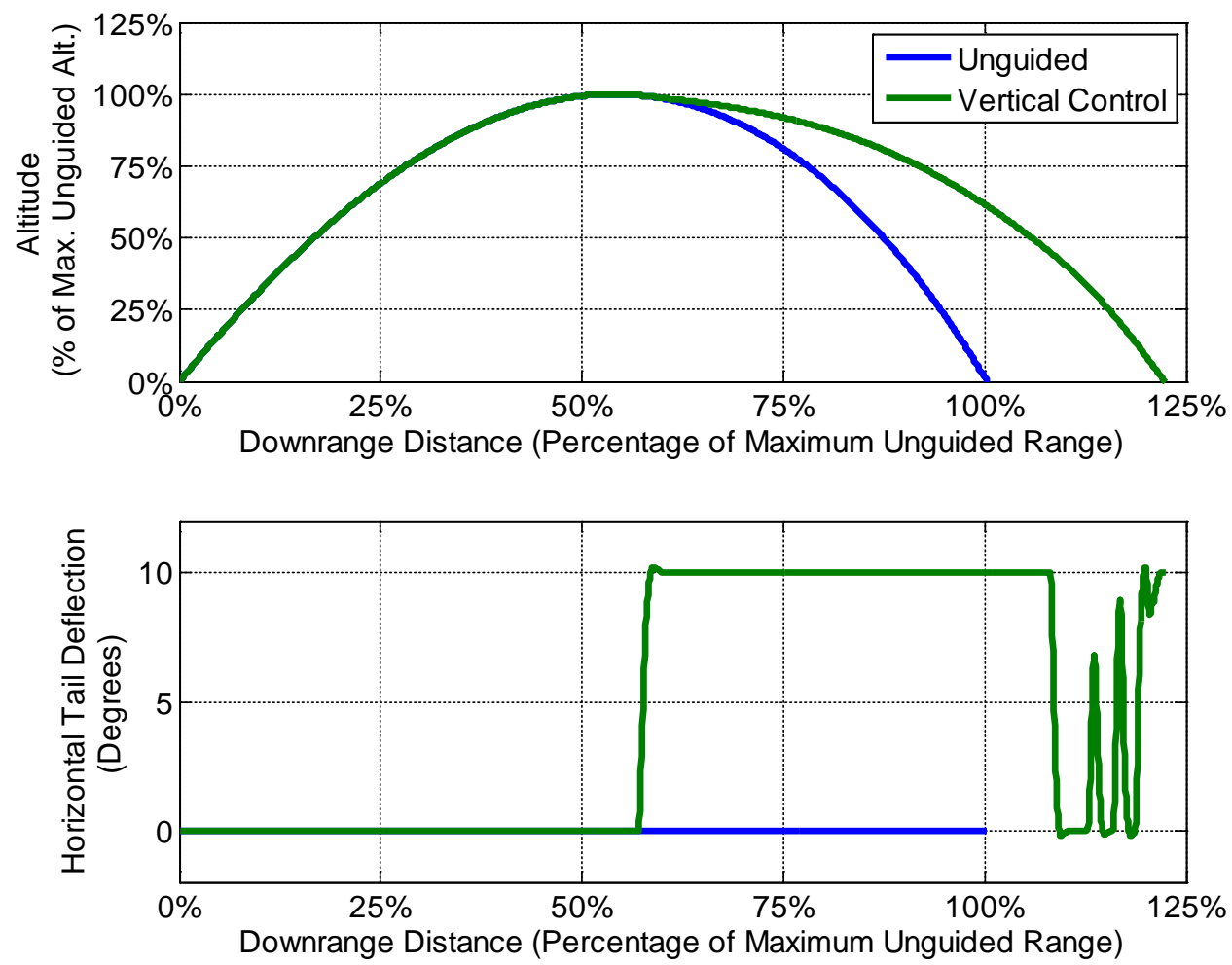

Figure 4-38: Longitudinal Target Distance $=122.5 \%$ Max Unguided Altitude $=0 \%$ Max Unguided
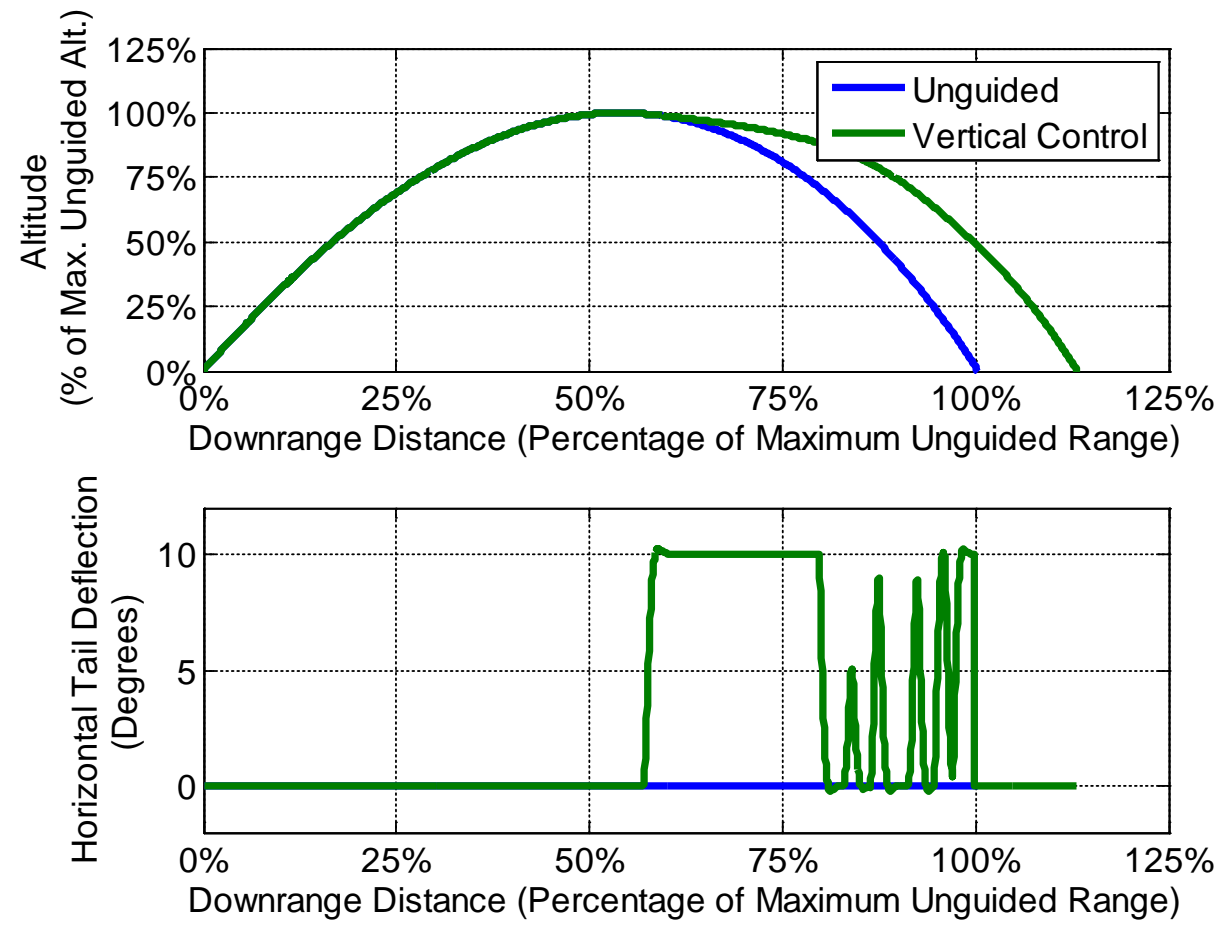

Figure 4-39: Longitudinal Target Distance $=100 \%$ Max Unguided Altitude $=50 \%$ Max Unguided 
Table 4-8: Results from Longitudinal Control Simulations

\begin{tabular}{|c|c|c|c|}
\hline Desired Distance & Desired Altitude & $\begin{array}{c}\text { Actual Distance at } \\
\text { Desire Altitude }\end{array}$ & Distance Offset \\
\hline $\begin{array}{c}\text { 122.5\% of Unguided } \\
\text { Max Range }\end{array}$ & $\begin{array}{c}\text { 0\% of Max Unguided } \\
\text { Altitude }\end{array}$ & $\begin{array}{c}122.39 \% \text { of Unguided } \\
\text { Max Range }\end{array}$ & $\begin{array}{c}11.3 \text { projectile } \\
\text { diameters }\end{array}$ \\
\hline $\begin{array}{c}100 \% \text { of Unguided Max } \\
\text { Range }\end{array}$ & $\begin{array}{c}50 \% \text { of Max Unguided } \\
\text { Altitude }\end{array}$ & $\begin{array}{c}99.8 \% \text { of Unguided } \\
\text { Max Range }\end{array}$ & $\begin{array}{c}22.5 \text { projectile } \\
\text { diameters }\end{array}$ \\
\hline
\end{tabular}

\subsection{Guidance Simulation Results}

Now that all three controllers have been designed and tested individually, the guidance capability of the $40 \mathrm{~mm} \mathrm{HP}$ was simulated. In the first test, all three controllers' interaction with each other was investigated. Their interaction was investigated by simulating the projectile with an initial roll rate of 62.5 $\mathrm{Hz}$ and having the controller de-spin, roll-level and guide the HP to a desired point of impact. The desired impact point simulated was $100 \%$ of the maximum unguided range downrange, at $28.3 \%$ of the maximum unguided altitude and 1500 projectile diameters right offset from the Line of Fire. Figure 4-40 shows the distance, altitude and line-of-fire offset of the flight path as well as the roll angle and the deflections made by the each set of tails. Results of this simulation show the projectile was off of the desire impact location by 14.3 projectile diameters.
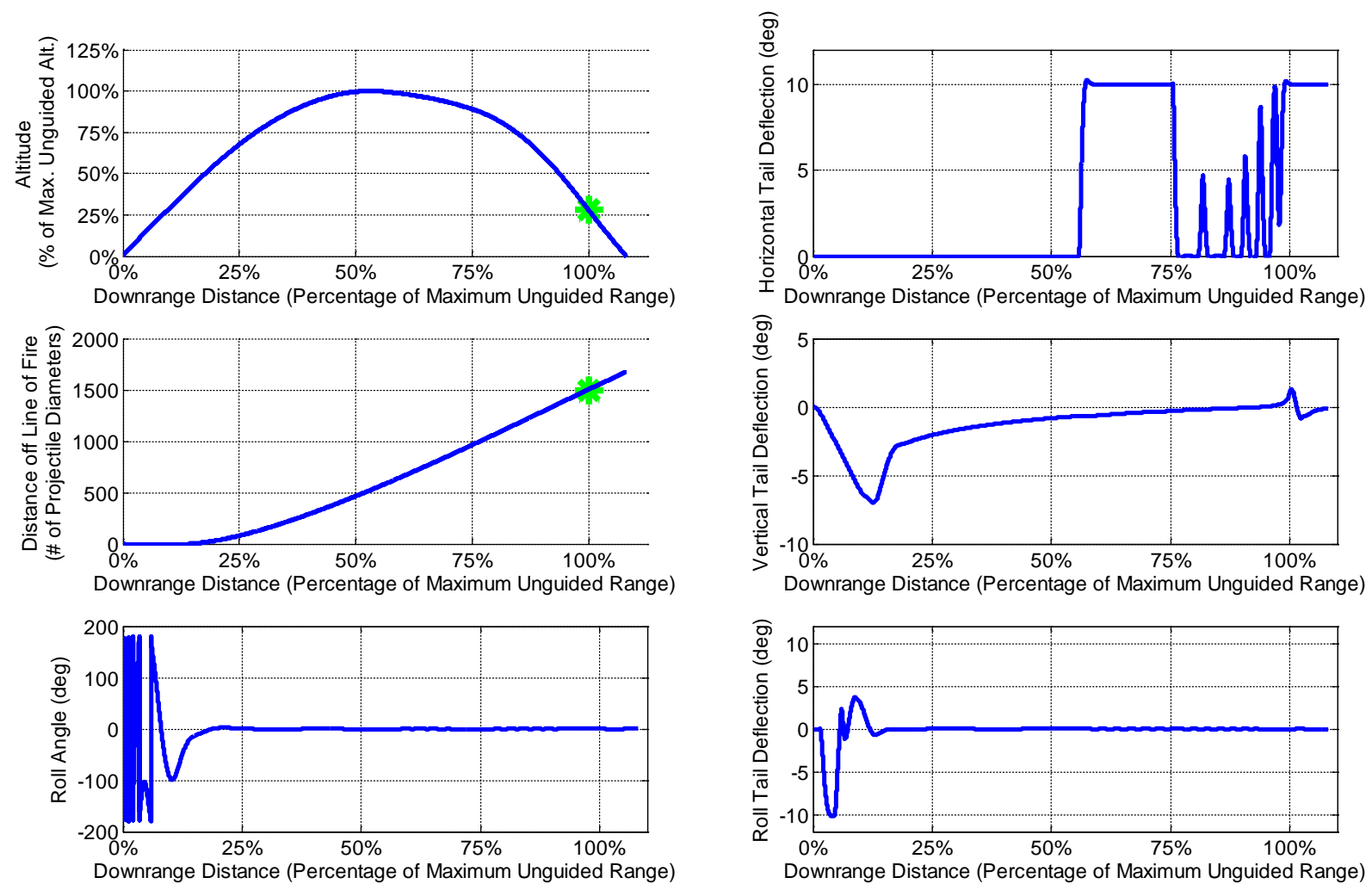

Figure 4-40: Simulation of Three Controllers Combined 
After showing that the three controllers could work together to de-spin, roll-level and guide the $\mathrm{HP}$ an achievable impact area was determined with the initial roll rate of the HP as zero degrees per second. This assumption was made because in the de-spin and roll leveling test, the projectile was able to become roll-leveled in $5 \%$ of the flight time. Therefore the majority of the flight is in a roll-level state. The roll-leveling controller was still utilized, but only to hold the projectile level during combined yaw and pitch maneuvers. The achievable impact locations were determined by simulating one location at a time for a grid of desired impact point. Figure 4-41, shows the distance between the desired impact point and actual impact point for each desired landing location. In Figure 4-41 the blue line indicates the projectiles location as the longitudinal controller was turned on, as it reaches -3.3 degrees. In this simulation the heading controller was turned on at launch.

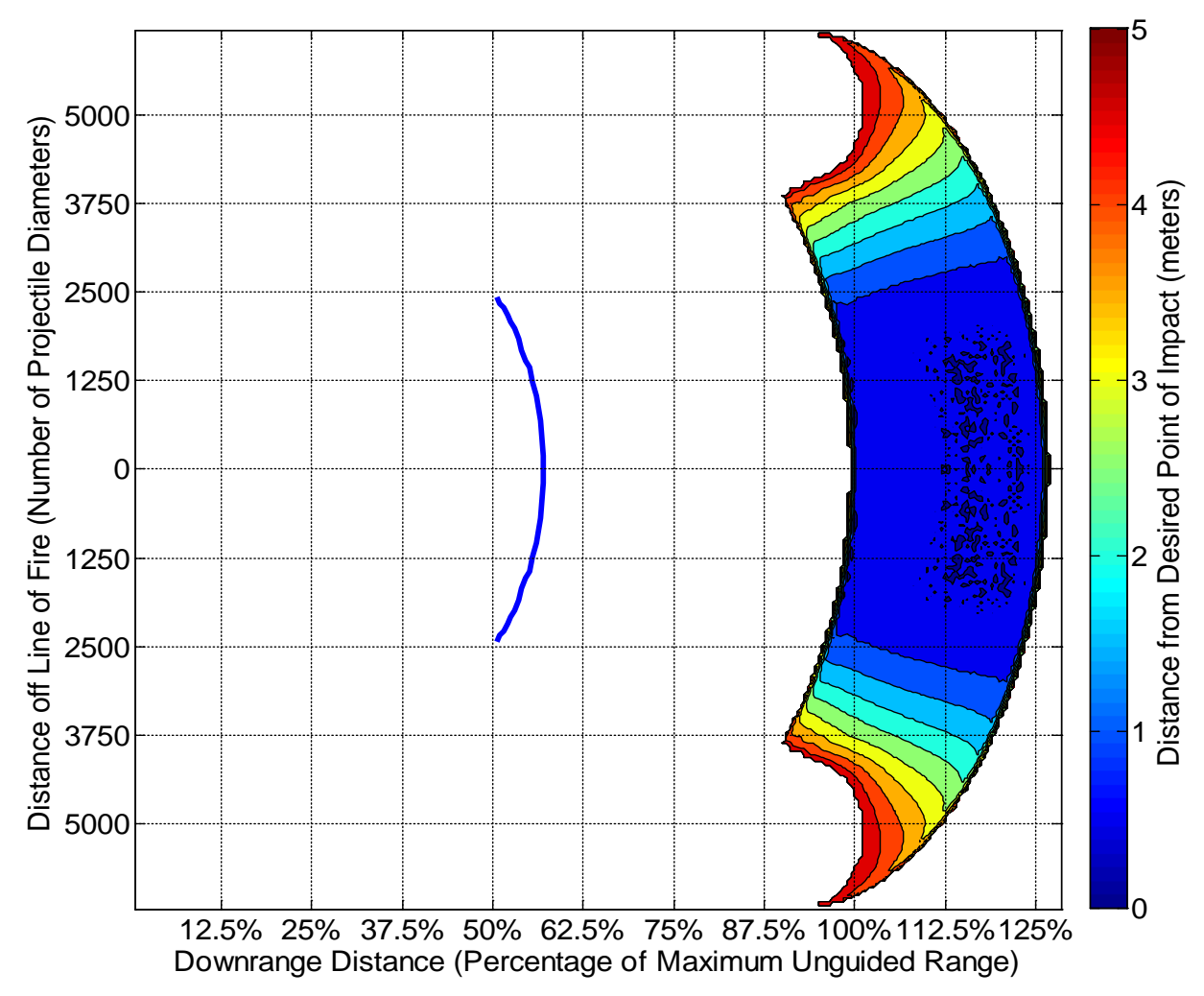

Figure 4-41: Targeting Landing Locations 
From the simulation it was seen that the range was extended $25.8 \%$ along the line of fire and was also able to turn up to 6250 projectile diameters off the line of fire. In Figure 4-41 it was seen the accuracy decreased towards the outer target areas. A greater error could occur in the outer ranges of desired impact points since the drag and lift data estimation calculated in Section 4.5.4 were different than the actual values. These differences could occur from vertical tail contributions that were not applied when estimation the drag and lift coefficients.

When considering a projectile like a $40 \mathrm{~mm} \mathrm{HP}$ it was important to know that the impact velocity has not been greatly changed from that of the original non-guided munition. The kinetic energy of the projectile upon impact causes a particular fragmentation pattern. By decreasing the kinetic energy in which the projectile strikes the ground, the fragmentation pattern changes from the designed pattern. Figure 4-42 shows the impact velocity for the projectile during the same simulation test as Figure 4-41, where the colorbar depicts the velocity as a percentage of the impact velocity for the unguided simulation.

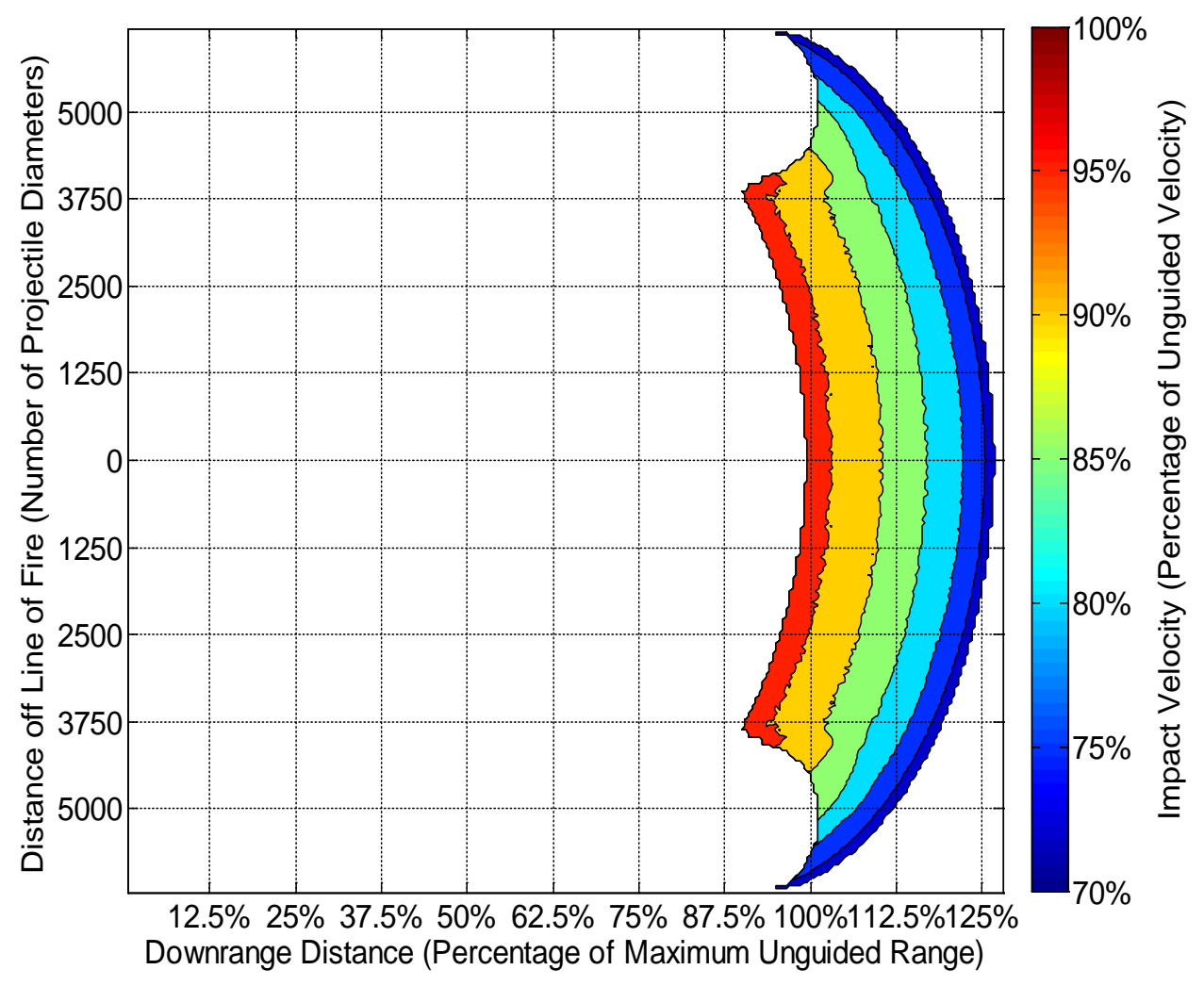

Figure 4-42: Final Velocity of the Projectile 
The amount of tail control required for each desired impact location was also an important characteristic of the guided flight to be shown. By knowing the areas in which maximum deflection magnitude was necessary the desired impact point area could be narrowed for a gust situation. If the projectile takes maximum tail deflection to get to a specific impact point in a gust free environment, it is not realistic during an actual launch since any gust it needed to control against would cause the projectile to not be able to achieve its point of impact. In Figure 4-43, the tail deflection average magnitude shows the areas where the most tail deflection was required to hit its desired point of impact. The deflection magnitude was calculated as the average magnitudes of the vertical and horizontal tail deflections. The colorbar of Figure 4-43 shows the magnitude of tail deflection, in degrees, required for each desired point of impact.

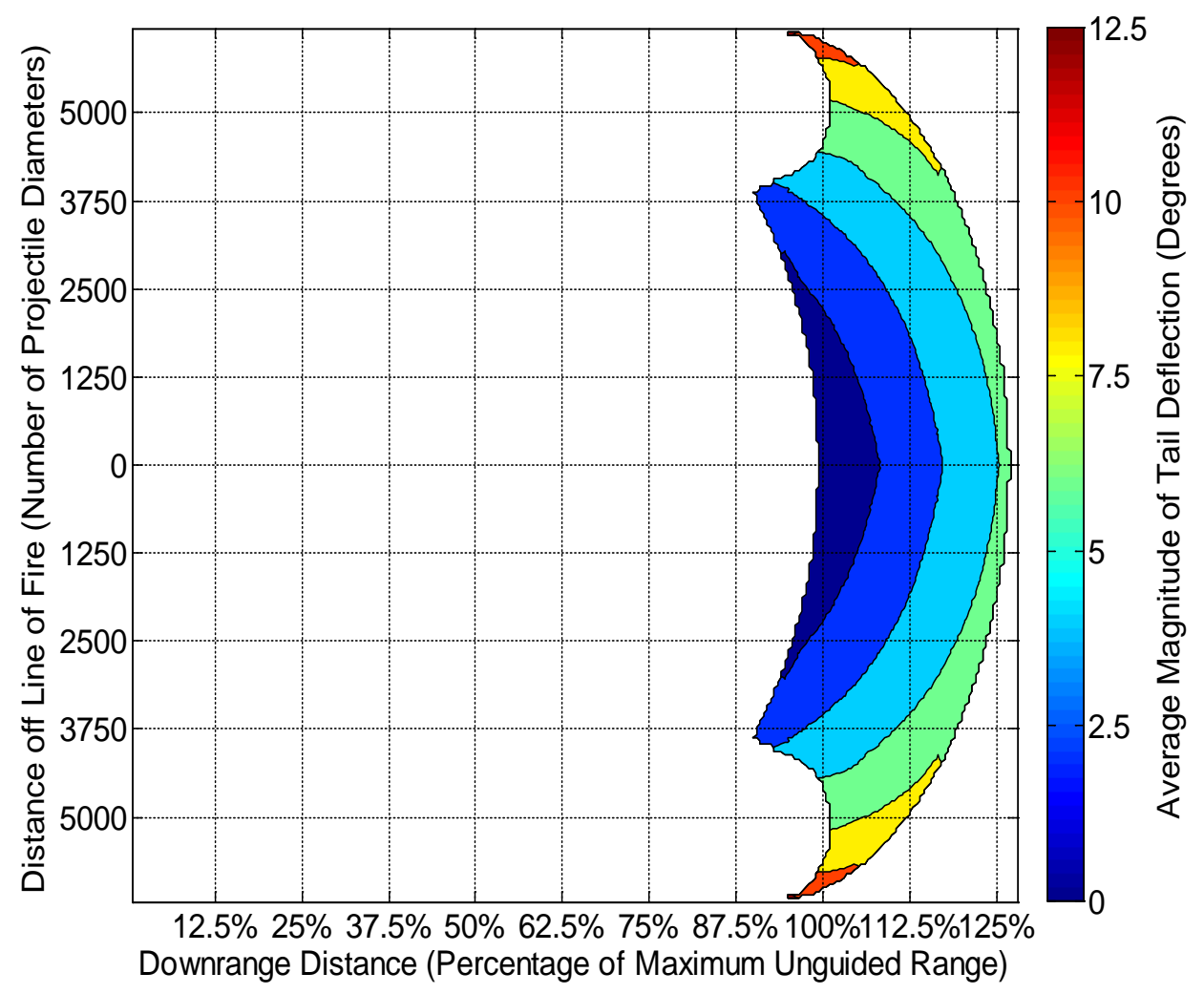

Figure 4-43: Magnitude of Average Tail Deflections

The M781 projectile was modified with a cruciform tail section as described in Section 3.4 then modeled using an aerodynamic build-up. Each of the four tails had an adjustable angle of attack and was modeled with a servo delay. With the use of a de-spin and roll-leveling controller the tails were deflected to steadily de-spin and roll-level the HP in 5\% of its flight time. Once the HP was roll-leveled, guidance maneuvering was begun. A heading controller was created to maneuver the HP to a particular heading 
angle or distance from the line of fire using its two vertical tails. Longitudinal control was achieved with a controller which deflected the HP's horizontal tails to 10 degrees then back to zero as the HP's ballistic trajectory crossed its desired downrange distance and altitude. The three controllers combined were simulated and results showed that the HP could be de-spun, roll-leveled and guided to 0.57 meters of its desired point of impact. When the HP was not spinning at launch the effect of the longitudinal and heading controllers were simulated to determine an area of achievable impact points. Simulation results show that the HP could be guided to impact points up to 25\% beyond the range of the baseline M781 and 6250 projectile diameters off the line of fire.

\subsection{Disturbance Rejection of Controllers}

An understanding of disturbances encountered, such as a side gust during guidance, is critical to ensure that the desired flight was still achieved. Disturbance rejection for guidance control was analyzed by adding a cross wind vector to the freestream velocity vector in the simulation.

Two cases of wind were investigated with multiple wind velocities to determine change in distance offset to the desired point of impact from the wind free case. The first case consists of a two second wind gust occurring between two and four seconds of flight time. The wind gust velocities over a two second gust were chosen to be two, five and ten meters per second. The gust duration was chosen from an average gust time found in (Beljaars 1987). The three velocity values were chosen to show how increasing velocity effected guidance. Also a constant sustained velocity wind case was investigated for two, five and ten meters per second sustained wind throughout the entire flight. The wind was simulated to be blowing normal to the line of fire from left to right. The wind velocity in this case was applied normal to the line of fire and needed to be transformed from earth fixed to body coordinate system using the horizontal wind model block in the aerospace toolbox of Simulink. 
Three points of impact were chosen as the desired locations for the wind gust and sustained wind cases. The desired impact points were compiled in Table 4-9. Location 1, 105\% max unguided range and 5000 projectile diameters left, was chosen because it is an achievable impact location which was on the far left end of the impact grid and to reach this location the projectile would be going into the direction of the wind. Location 2, 105\% max unguided range and 5000 projectile diameters right, was chosen because it was an achievable impact location on the far right of the impact grid and in the same direction as the wind. Determining if the HP could hold on the line of fire and reach a point with no offset was shown by choosing Location 3, 125\% max unguided range. The ground tracks for each case were shown in Figure 4-44 through Figure 4-49 to show how the projectile deviated from its trajectory with and without wind while being guided to the desired impact location. The percentage difference for each case with wind was calculated using equation (4-10), where offset with wind $\left(\mathrm{Off}_{\text {Wind }}\right)$ was compared with the windless impact offset ( Off $\left._{\text {NoWind }}\right)$.

Table 4-9: Desired Impact Locations for Disturbance Rejection

\begin{tabular}{|c|c|c|}
\hline $\begin{array}{c}\text { Location } \\
\text { Number }\end{array}$ & $\begin{array}{c}\text { Desired Down Range } \\
\text { Distance }\end{array}$ & $\begin{array}{c}\text { Desired Location off Line of } \\
\text { Fire }\end{array}$ \\
\hline 1 & $105 \%$ Max Unguided Range & 5000 Projectile Diameters Left \\
\hline 2 & $105 \%$ Max Unguided Range & 5000 Projectile Diameters Right \\
\hline 3 & 125\% Max Unguided Range & On-line \\
\hline
\end{tabular}

$$
\left(\frac{O f f_{\text {Wind }}-O f f_{\text {NoWind }}}{O f f_{\text {NoWind }}}\right) \times 100 \%
$$




\subsubsection{Two Second Wind Gust}

The case in which a two second wind gust was added to the model was simulated first to predict the performance due to different gust velocities. The first simulation in this case was used to predict the performance of the HP when the desired impact location required the HP to fly into the wind gust. The first location desired was for the HP to land at 105\% maximum unguided range downrange and 5000 projectile diameters left off the line of fire. When the HP was simulated without wind gust it was able to land 104 projectile diameters off of the desired point of impact. As the wind velocity was increased the projectile was simulated to land up to $18 \%$ farther from the desired location, Figure 4-44.

Table 4-10: Impact offset due to Wind Gust to Desired Location 1

\begin{tabular}{|c|c|c|}
\hline $\begin{array}{c}\text { Wind Speed } \\
(\mathrm{m} / \mathrm{s})\end{array}$ & $\begin{array}{c}\text { Distance Off Desired } \\
\text { Impact } \\
\text { (projectile diameters) }\end{array}$ & $\begin{array}{c}\text { \% Difference } \\
\text { from } 0 \mathrm{~m} / \mathrm{s}\end{array}$ \\
\hline 0 & 104 & $3 \%$ \\
\hline 2 & 107.5 & $8 \%$ \\
\hline 5 & 112.5 & $18 \%$ \\
\hline 10 & 122.5 & \\
\hline
\end{tabular}

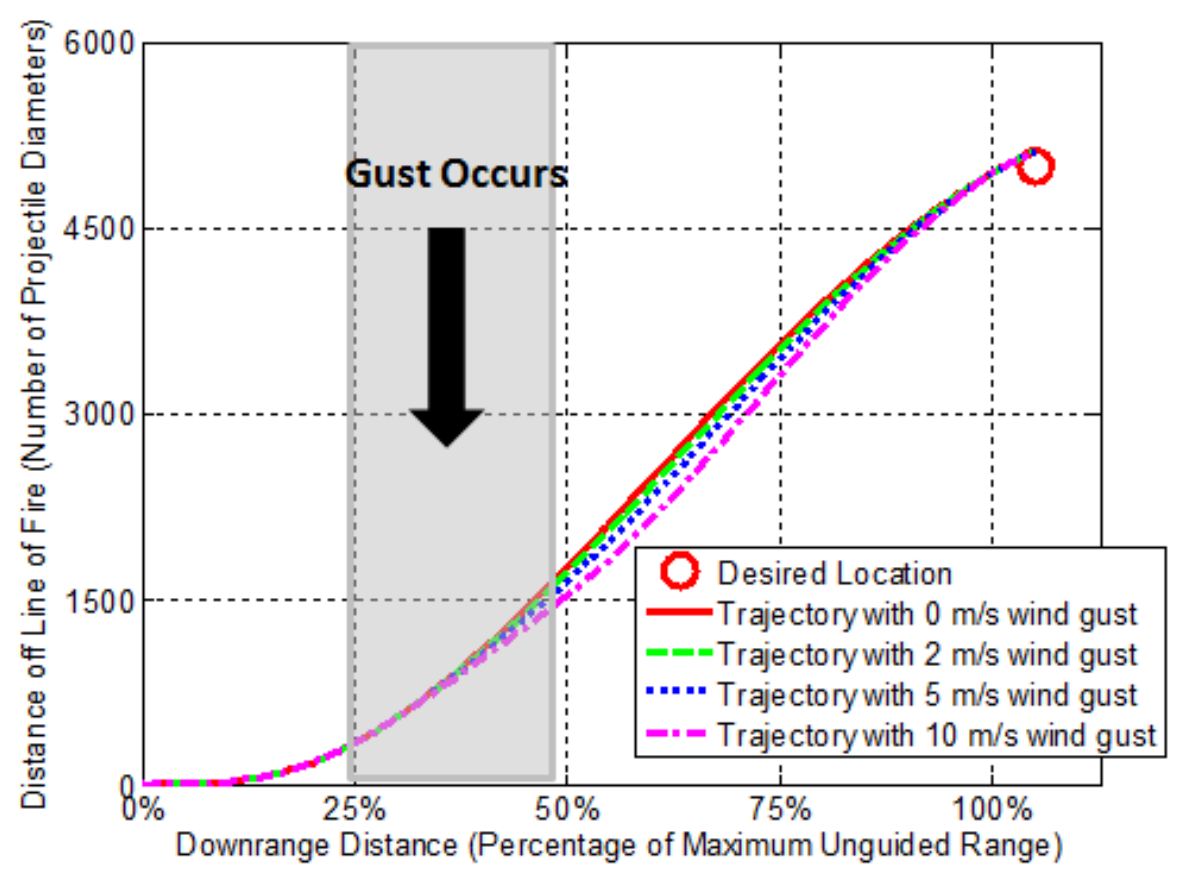

Figure 4-44: Ground Track of HP with Wind Gust to Desired Location 1 
The second simulation in this case was used to predict the performance of the HP when the desired impact location required the HP to fly with the wind gust. The second location desired was for the HP to land at $105 \%$ maximum unguided range downrange and 5000 projectile diameters right off the line of fire. When the HP was simulated without wind gust it was able to land 104 projectile diameters off of the desired point of impact. As the wind velocity was increased the projectile was simulated to land up to $10 \%$ closer to the desired location, Figure 4-45.

Table 4-11: Impact offset due to Wind Gust to Desired Location 2

\begin{tabular}{|c|c|c|}
\hline $\begin{array}{c}\text { Wind Speed } \\
(\mathrm{m} / \mathrm{s})\end{array}$ & $\begin{array}{c}\text { Distance Off Desired } \\
\text { Impact } \\
\text { (projectile diameters) }\end{array}$ & $\begin{array}{c}\text { \% Difference } \\
\text { from } 0 \mathrm{~m} / \mathrm{s}\end{array}$ \\
\hline 0 & 104 & $-3 \%$ \\
\hline 2 & 101 & $-7 \%$ \\
\hline 5 & 96.5 & $-10 \%$ \\
\hline 10 & 93.5 & \\
\hline
\end{tabular}

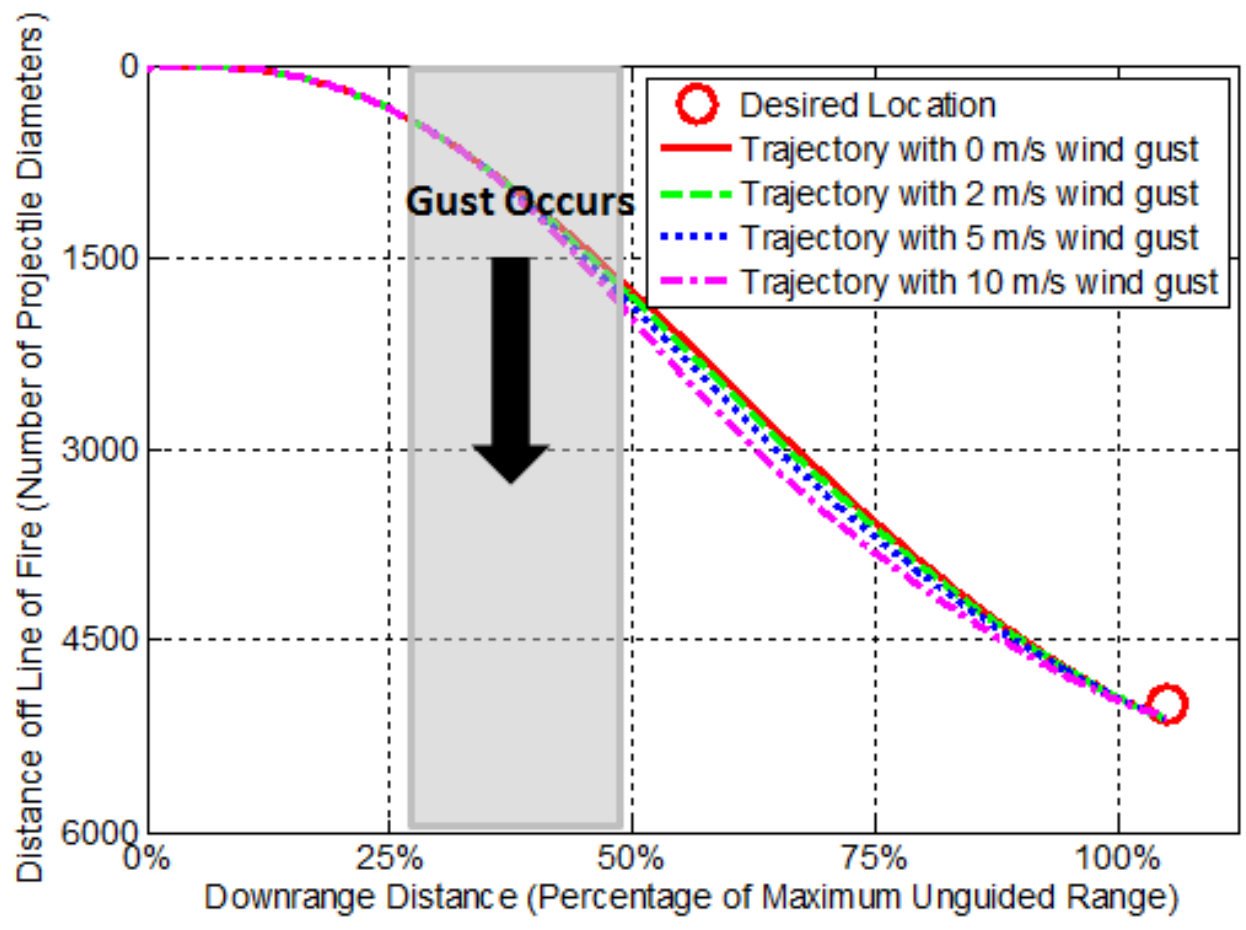

Figure 4-45: Ground Track of HP with Wind Gust to Desired Location 2 
The final simulation in this case was used to predict the performance of the HP when the desired impact location required the HP remain on the line of fire. The third location desired was for the HP to land at $125 \%$ maximum unguided range downrange on the line of fire. When the HP was simulated without wind gust it was able to land 14.3 projectile diameters off of the desired point of impact. As the wind velocity was increased the projectile was simulated to land up to $44 \%$ farther from the desired location, Figure 4-46.

Table 4-12: Impact offset due to Wind Gust to Desired Location 3

\begin{tabular}{|c|c|c|}
\hline $\begin{array}{c}\text { Wind Speed } \\
(\mathrm{m} / \mathrm{s})\end{array}$ & $\begin{array}{c}\text { Distance Off Desired } \\
\text { Impact } \\
\text { (projectile diameters) }\end{array}$ & $\begin{array}{c}\text { \% Difference } \\
\text { from } 0 \mathrm{~m} / \mathrm{s}\end{array}$ \\
\hline 0 & 14.3 & $14 \%$ \\
\hline 2 & 16.3 & $35 \%$ \\
\hline 5 & 19.3 & $44 \%$ \\
\hline 10 & 20.5 & \\
\hline
\end{tabular}

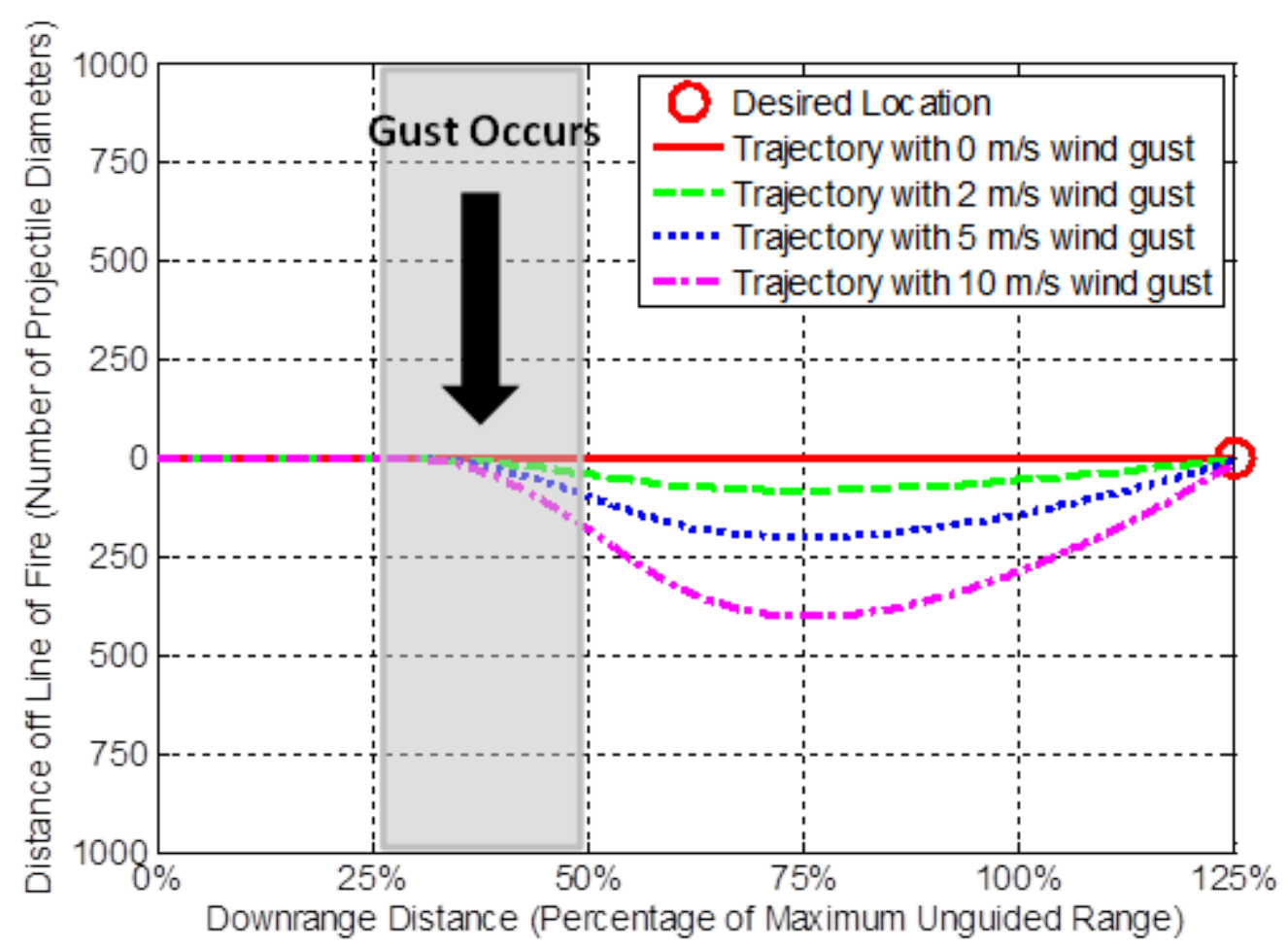

Figure 4-46: Ground Track of HP with Wind Gust to Desired Location 3 


\subsubsection{Sustained Cross Wind}

The second case of sustained wind was added to the model and used to predict the performance of the HP due to different sustained wind velocities. The first simulation in this case was used to predict the performance of the HP when the desired impact location required the HP to fly into the sustained wind. The first location desired was for the HP to land at 105\% maximum unguided range downrange and 5000 projectile diameters left off the line of fire. When the HP was simulated without wind it was able to land 104 projectile diameters off of the desired point of impact. As the wind velocity was increased it was seen that with wind of two meters per second the projectile was simulated to land up to $62 \%$ closer to the desired location. However, as the wind velocity was increased up to ten meters per second the HP was simulated to land 961\% farther from desired, Figure 4-47.

Table 4-13: Impact offset due to Sustained Wind to Desired Location 1

\begin{tabular}{|c|c|c|}
\hline $\begin{array}{c}\text { Wind Speed } \\
(\mathrm{m} / \mathrm{s})\end{array}$ & $\begin{array}{c}\text { Distance Off Desired Impact } \\
\text { (projectile diameters) }\end{array}$ & $\begin{array}{c}\text { \% Difference } \\
\text { from 0 m/s }\end{array}$ \\
\hline 0 & 104 & $-62 \%$ \\
\hline 2 & 39.3 & $64 \%$ \\
\hline 5 & 171 & $961 \%$ \\
\hline 10 & 1104 & \\
\hline
\end{tabular}

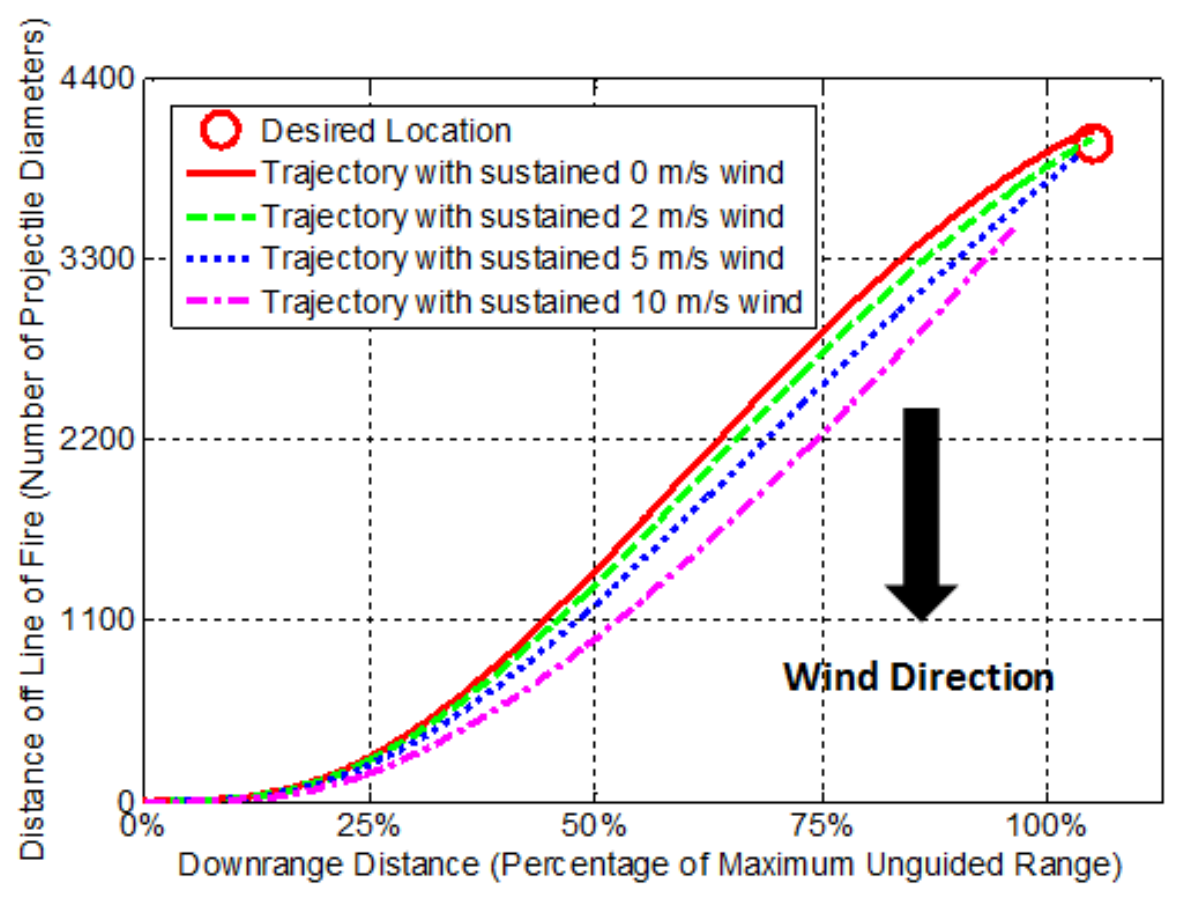

Figure 4-47: Ground Track of HP with Sustained Wind to Desired Location 1 
The second simulation in this case was used to predict the performance of the HP when the desired impact location required the HP to fly in the same direction as the sustained wind. The second location desired was for the HP to land at 105\% maximum unguided range downrange and 5000 projectile diameters right off the line of fire. When the HP was simulated without wind it was able to land 104 projectile diameters off of the desired point of impact. As the wind velocity was increased the projectile was simulated to land up to 552\% farther from the desired location, Figure 4-48.

Table 4-14: Impact offset due to Sustained Wind to Desired Location 2

\begin{tabular}{|c|c|c|}
\hline $\begin{array}{c}\text { Wind Speed } \\
(\mathrm{m} / \mathrm{s})\end{array}$ & $\begin{array}{c}\text { Distance Off Desired } \\
\text { Impact } \\
\text { (projectile diameters) }\end{array}$ & $\begin{array}{c}\text { \% Difference } \\
\text { from } 0 \mathrm{~m} / \mathrm{s}\end{array}$ \\
\hline 0 & 104 & $86 \%$ \\
\hline 2 & 193.3 & $239 \%$ \\
\hline 5 & 353 & $552 \%$ \\
\hline 10 & 678.3 & \\
\hline
\end{tabular}

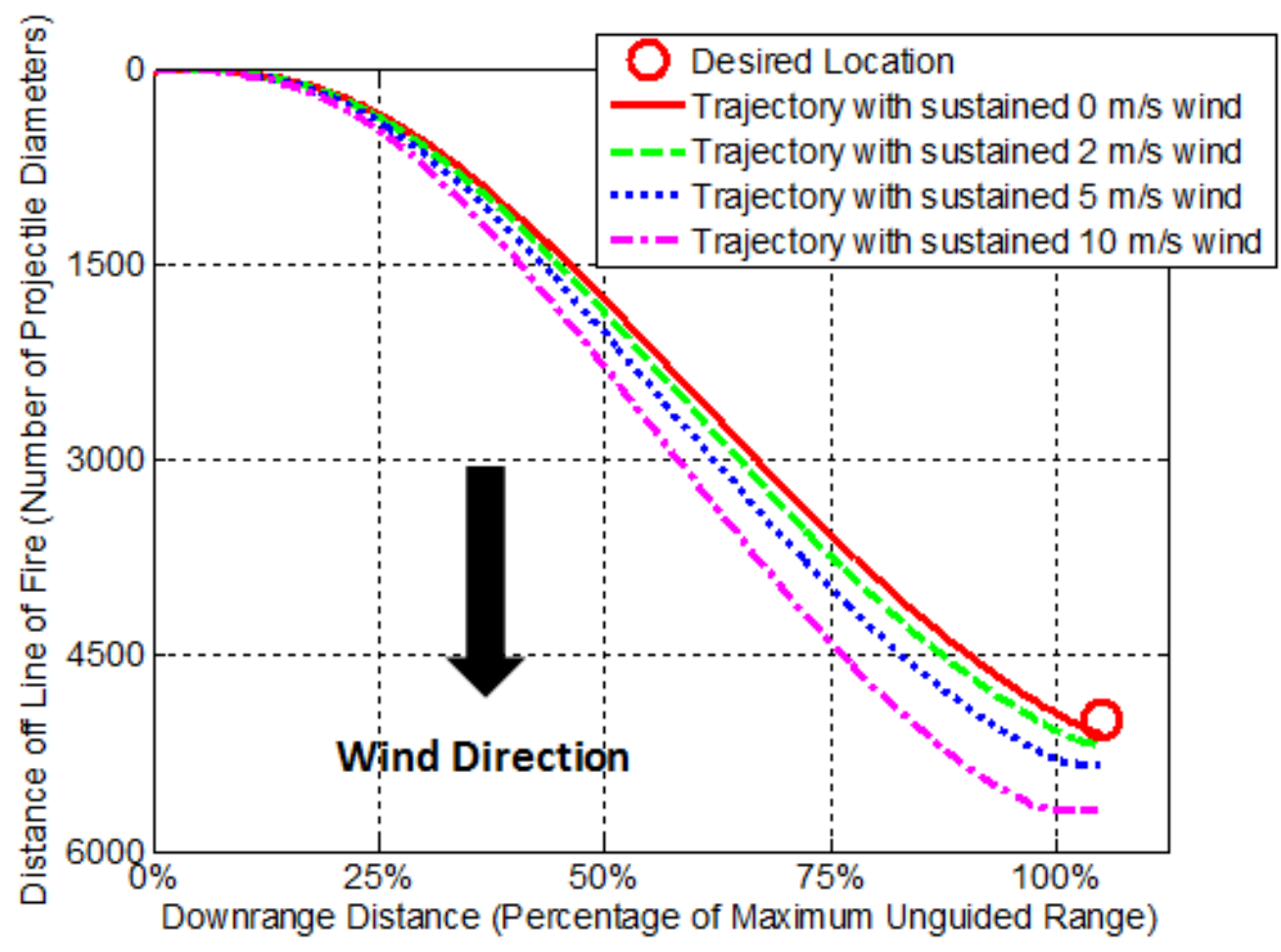

Figure 4-48: Ground Track of HP with Sustained Wind to Desired Location 2 
The final simulation in this case was used to predict the performance of the HP when the desired impact location required the HP to remain on the line of fire. The third location desired was for the HP to land at $125 \%$ maximum unguided range downrange and on the line of fire. When the HP was simulated without wind it was able to land 14.3 projectile diameters off of the desired point of impact. As the wind velocity was increased the projectile was simulated to land up to $6433 \%$ farther from the desired location, Figure 4-49.

Table 4-15: Impact offset due to Sustained Wind to Desired Location 3

\begin{tabular}{|c|c|c|}
\hline $\begin{array}{c}\text { Wind Speed } \\
(\mathrm{m} / \mathrm{s})\end{array}$ & $\begin{array}{c}\text { Distance Off Desired } \\
\text { Impact } \\
\text { (projectile diameters) }\end{array}$ & $\begin{array}{c}\text { \% Difference } \\
\text { from } 0 \mathrm{~m} / \mathrm{s}\end{array}$ \\
\hline 0 & 14.3 & $679 \%$ \\
\hline 2 & 111 & $2218 \%$ \\
\hline 5 & 330.3 & $6433 \%$ \\
\hline 10 & 931 & \\
\hline
\end{tabular}

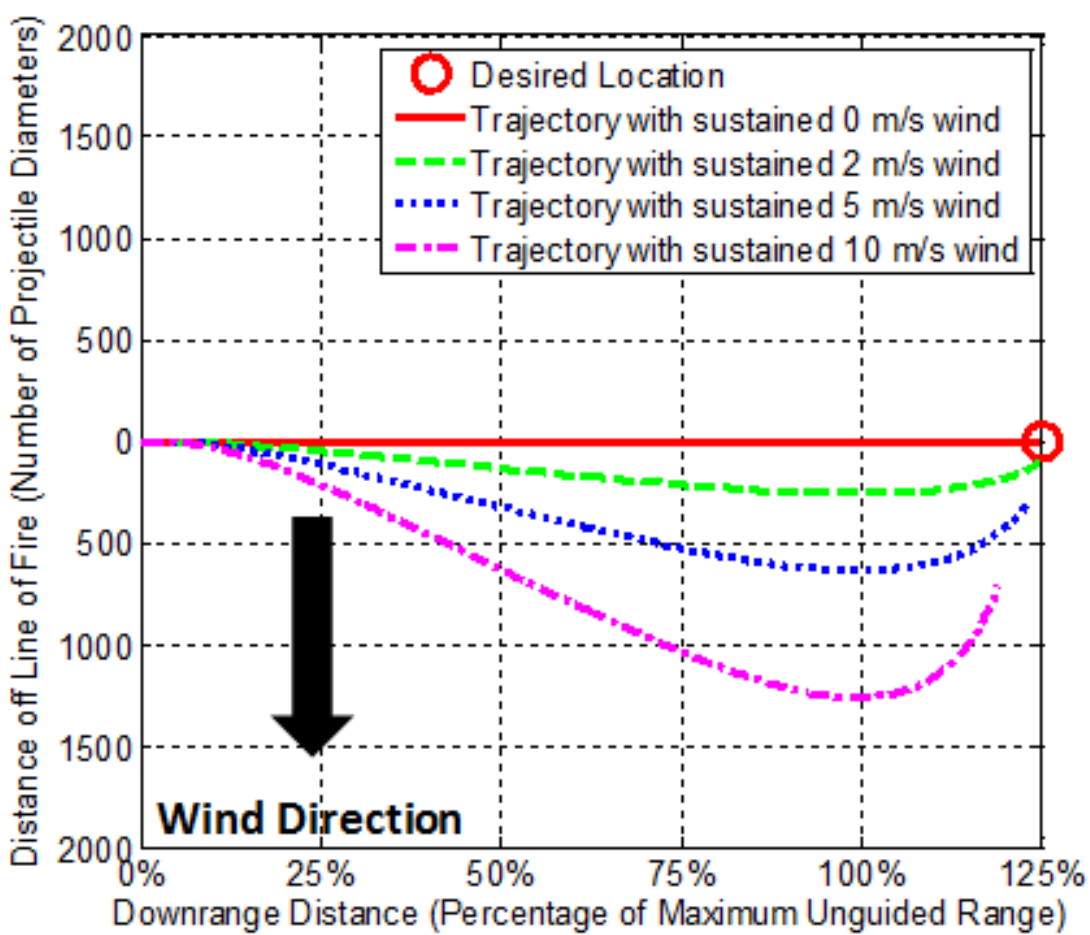

Figure 4-49: Ground Track of HP with Sustained Wind to Desired Location 3 
Results from the wind disturbance simulations showed that the accuracy was reduced when wind occurred for each desired location and both wind cases except the gust case for the desired location 2. This case resulted in the HP being closer to the desired point of impact because the gust helped to push the HP towards the desired impact point. Even though accuracy was decreased to guidance controller was still able to maneuver the HP towards the desired location. Viewing the ground tracks of Figure 4-47 and Figure 4-49, it was seen that the HP did not miss the desired point of impact by being off line but it ended up being short in most cases. This was because the HP did not have a propulsion system and when flying into the wind the projectile had a greater amount of drag, reducing range. 


\section{Chapter 5 Conclusions and Future Work}

\subsection{Summary}

In this thesis, a method was sought to use deflectable control surfaces in combination with a guidance system to maneuver a HP to a desired impact point, while maintaining stability and using the body as a lifting surface. This method was then used to adequately size tail surfaces on a M781 $40 \mathrm{~mm}$ round to test stability could be ensured. The M781 is a spin-stabilized projectile that needs to be roll-level before guidance could be employed so that the body could be pitched up and used as a lifting surface. Pitch, roll and yaw controllers were thus required to deflect the local angle of attack of the tail surfaces to de-spin and roll-level the HP before maneuvering it to a desired impact point.

The process of creating a control method was begun by collecting aerodynamic data and specifications from a $40 \mathrm{~mm}$ M781. A model was then created with the use of Simulink's aerospace toolbox to solve the equations of motion. Forces and moments were estimated due to aerodynamic coefficients. Then a build-up of aerodynamic coefficients was generated for the M781 equipped with tail surfaces. The tail surface size was determined to meet the de-spin requirement of being roll-leveled in less than 2 seconds and to ensure the HP was statically and dynamically stable. Tail control surfaces were chosen for this HP because of their tendency to stabilize a statically unstable projectile, by shifting the center of pressure aft of the center of gravity. A cruciform tail section with each tail having a chord of 20 $\mathrm{mm}$ and a span of $30 \mathrm{~mm}$ was required to have the projectile de-spin and roll-level in 1.64 seconds. The tail surface area also ensured that the HP was statically and dynamically stable.

A roll controller was developed next to deflect the tail surfaces to de-spin and roll-level the HP.

The roll controller was developed with two PID controllers; one was used to steadily reduce the roll rate and the second to roll-level the projectile. Each PID controller was tuned through an iterative process. An adaptive transition point between the two controllers was determined to reduce time required for the projectile to become roll-leveled.

An important step in this method was to determine technique to be used for guidance. Guidance was divided into two parts: longitudinal and heading control. Heading control was achieved through a PID feedback control system. Feedback control for heading maneuvers was used to deflect the vertical tails so the HP reached a desired distance off the line of fire or a specified heading azimuth. The PID gain values were determined for this controller by using the built in tuning function in Simulink. With the use of the heading controller, the HP was able to be maneuvered up to 6250 projectile diameters, about 33 degrees, off the line of fire and land within 125 projectile diameters of the desired impact point. A longitudinal controller was developed to deflect the horizontal tails to a maximum deflection of 10 degrees, nosing the body up. The HP was nosed up so that the body could be used as a lifting surface 
extending range. Next, the controller was used to deflect the horizontal tails back to zero degrees when the estimated trajectory of the projectile crossed the desired impact point. The longitudinal component of guidance was able to achieve an increase in range of $25.8 \%$ as compared to a standard unguided M781. By following the method described, a control system was determined for a specified tail surface geometry applied to a $40 \mathrm{~mm}$ M781 for stability and guidance.

The contribution of this work to the field of engineering was the use of a dual mode controller to de-spin and roll-level a HP and the use of decoupled heading and longitudinal control. Decoupled control was important so that the body could be used as a lifting surface for range extension and the tails could be used to maneuver the HP to a desired point of impact.

\subsection{Future Work}

In extension to the research conducted for the Hybrid Projectile project on the $40 \mathrm{~mm}$ munition a few additional tasks are recommended. First, the projectile designed in this study needs to be fabricated and flight tested to determine if the controllers designed in simulation will work in a real flight scenario. Further aerodynamic data should be investigated, through steady-state CFD and static experimental tests, for the combined geometry of both body and tails. Transient experimental tests could be used to verify the simulation results by using a wind tunnel and a spinning rig to test the de-spin controller. The wind tunnel could also be useful with load cell data to determine if forces and moments created by the tail surfaces are adequate for guidance.

Further development can also be done on the verification model of Section 3.3 in which this HP could have deflectable tails applied similar to the HP in Figure 5-1. This HP can be equipped with the Px4 autopilot and used to deflect the control surfaces. Adding control capability to the autopilot requires that the control algorithms from Matlab be discretized. Sensors from the autopilot can be used determine the states of the projectile and the autopilot also has output capabilities. Simulink can then be used to create a source code which can be loaded onto autopilot and used to deflect the control surfaces, guiding the HP. 


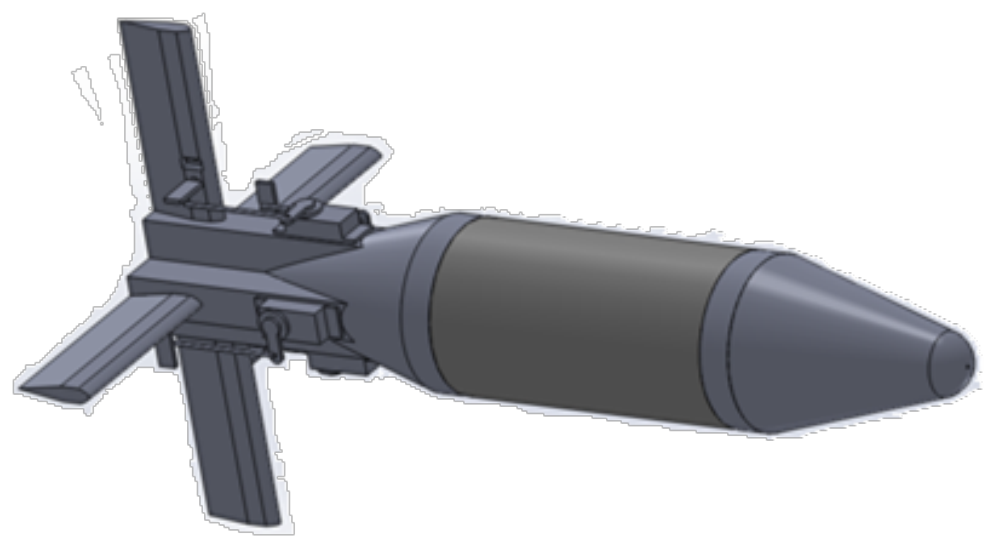

Figure 5-1: Example concept of Tail modification for a HP

Next, the stowable wing designs which have been investigated for the $40 \mathrm{~mm}$ HP could be applied for further range extension. With the tail surfaces designed the range was only increased by 25.8\%, but could be extended more with larger lifting surfaces. From a simple lift to drag ratio comparison the lift to drag ratio of the HP with the tails deflected was approximately 1.7:1 resulting in a glide slope of 30 degrees. However, with wings added the total lift to drag ratio could be closer to 4:1 with a glide slope of 14 degrees. If the wings are deployed at the same pitch angle as the tails were deflected the range could be extended at least 50\% beyond the standard unguided $40 \mathrm{~mm}$ M781 (Hamburg 2010). Wings also can add instability to the HP which can be corrected through the use of the method described in this thesis. Having a HP with wings and control surfaces allows for the projectile to be guided and achieve further range extension. 


\section{References}

ALS Technologies. "M781 Product Data Sheet". Bull Shoals, AR, Jun. 2009.

$<$ http://static.alstechnologies.com/specs/MEI/M781.pdf>.

Barrowman, James. Calculating the Center of Pressure of a Model Rocket. Technical Information Report - 33. Phoenix, Arizona: Centuri Engineering Company, n.d.

Beljaars, A. C. M. . "The Influence of Sampling and Filtering on Measured Wind Gusts." Journal of Atmospheric and Oceanic Technology (1987): 613-626. Web.

Carlucci, Donald E. and Sidney S. Jacobson. Ballistics: Theory and Design of Guns and Ammunition. Boca Raton, FL: CRC/Taylor \& Francis Group, 2008. Print.

Christiansen, Reed Siefert. Design of an Autopilot for Small Unmanned Aerial Vehicles. MS Thesis, Brigham Young University, 2004.

Cooke, Gary W. "40mm Low-Velocity Grenades." 25 Feb. 2010. <http://www.inetres.com/gp/military/infantry/grenade/40mm_ammo.html\#M781>.

Dupuis, Alain. "Aeroballistics Range Tests of a $40 \mathrm{~mm}$ Projectile." 23rd International Symposium on Ballistics. Tarragona, Spain, (2007). 705-712.

Exploration, Stellar. Stellar-Exploration. 4 June 2011. 2013. <http://www.stellarexploration.com/\#!40mm-guided-grenade>.

FAS Military Analysis Network. "M203 40mm Grenade Launcher." 22 February 2000. Federation of American Scientists. 22 March 2014. <http://www.fas.org/man/dod-101/sys/land/m203.htm>.

"Flight Equations with Drag." 28 July 2011. National Aeronaitics and Space Administration. 29 June 2013. <http://www.grc.nasa.gov/WWW/k-12/airplane/flteqs.html>.

Fresconi, F, et al. "Flight mechanics of a novel guided spin-stabilized concept." Proc. IMechE Vol. 226 Part G: J. Aerospace Engineering (2011): 327-340.

Fresoni, F and P Plostins. "Control mechanism strategies for spin-stabilized projectiles." Proc. IMechE Vol. 224 Part G: J. Aerospace Engineering (2011): 979-991.

Gioia, Christopher J. Hybrid Projectile Body Angle Estimation for Selectable Range Increase. MS Thesis, West Virginia University, 2013.

Gkritzapis, Dimitrios N.; Margaris, Dionissios P.; Panagiotopoulos, Elias E.; Kaimakamis, George, et al. "Prediction of the Impact Point for Spin and Fin Stabilized Projectiles." WSEAS Transactions on Information Science and Applications Volume 5. Issue 12 (2008): 1667-1676.

Gregorek, Gerald M. Aerodynamic Drag of Model Rockets. Penrose, Colorado: Estes Industries, Inc., 1970.

Haiyang Chao, Yongcan Cao, YangQuan Chen. "Autopilots for Small Fixed-Wing Unmanned Air Vehicles: A Survey." IEEE International Conference on Mechatronics and Automation. Harbin, China, 2007. 3144-3149.

Hamburg, Shanti. Conceptual and Preliminary Design of a Stowable Ruggedized Micro Air Vehicle. MS Thesis, West Virginia University, 2010.

Hasnink, Kelly. "Mortar Guidance Kit (MGK)." 2010 Joint Armaments Conference. 2010. <http://www.dtic.mil/ndia/2010armament/TuesdayCumberlandKellyHanink.pdf>.

Ives, John M. "Armored Cavalry Mortars: Operations and Myths." ARMOR November-December 2001. 22-26.

Jie, Cheng; Xiaoming, Wang; Jiyan, Yu; Wenjin, Yao. "On the Response of Spin Stabilized Projectile to Side Force by Angular Motion and Impact." 27th International Symposium on Ballistics. Freiburg, Germany: DEStech Publications, Inc., 2013. 225-232. 
Langdon, S.D and W.V. Cross. Fixed Wing Stability and Control: Theory and Flight Test Techniques. Defense Technical Information Center, 1977.

Larsen, Erik, et al. Ranger Knowledge: The All-Inclusive Study Guide for Rangers. Manhattan: St. Martins Press, 2013.

Lovas, Andre, et al. DARPA SCORPION Program Transition to Army Lethality ATO Program: A Success Story. Picatinny: ARL, 2009.

Lyon, David H. "Development of a 40mm Nonlethal Cartridge." DT/FL Research Journal (1997).

Manole, L; Logsdon, E; Palathingal, M; Sebasto, A. Gun launched hybrid projectile. United States of America: Patent 8115149. 21 Jul 2009.

Martinez, Marcelo, Nicolas Bruno and Dr. Roy Kelly. "27th International Symposium on Ballisitcs." 27th International Symposium on Ballisitcs. Freiburg, Germany: DEStech Publications. Inc, 2013. 302-313.

Matlab. "MATLAB R2013a (8.1.0.604)." Products and Services Online. The MathWorks Inc., 2014. $<$ http://www.mathworks.com/products/?s_tid=gn_ps>.

McMichael, J; Lovas, A; Plostins, P; Sahu, J; Brown, G; Glezer, A. "Microadaptive Flow Control Applied to a Spinning Projectile." 2nd AIAA Flow Control Conference. Portland, Oregon: AIAA, 2004. 30.

Milinovic, Momcilo; Jerkovic, Damir; Jeremic, Olivera; Kovac, Mitar. "Experimental and Simulation Testing of Flight Spin Stability for Small Caliber Cannon Projectile." Strojniški vestnik - Journal of Mechanical Engineering (2012): 394-402.

MLM International. 40 mm Low Velocity M781 Practice Grenade. Washington Grove, MD, 2005. $<$ http://www.mlmintl.com/40mmM781.pdf>.

Niskanen, Sampo. Development of an Open Source model rocket simulation. MS Thesis, Helsinki University of Technology, 2009.

Phillips, Warren F. Mechanics of Flight 2nd Edition. Hoboken, NJ: John Wiley \& Sons, Inc., 2010.

PX4 Autopilot. n.d. 24 September 2013. <https://pixhawk.ethz.ch/px4/modules/px4fmu>.

Ramezani, Roozbeh Falah. Non-linear Modeling and Control of Unmanned Aerial Vehicle. MS Thesis, American University of Sharjah, 2012.

Raymer, Daniel P. Aircraft Design: A Conceptual Approach. Reston, Virginia: American Institute of Aeronautics and Astronautics, Inc., 1999. Print.

Roskam, Jan. Aircraft Flight Dynamics and Automatic Flight Controls. Lawrence, KS: DARcorporation, 2003. Print

Roskam, Jan. Methods for Estimating Stability and Control Derivatives of Conventional Subsonic Planes. Lawrence, Kansas: University of Kansas, 1971. Print.

"Torayca." 26 November 2002. T300 Data Sheet. 27 March 2014. $<$ http://www.toraycfa.com/pdfs/t300datasheet.pdf $>$.

Vogel, William L. Numerical Flight Testing of a Tube-launched Transformable Micro Air Vehicle. MS Thesis, West Virginia University, 2012.

White, Frank M. Viscous Fluid Flow Third Edition. New York, NY: The McGraw-Hill Companies, Inc., 2006. Print.

Wilhelm, Jay P.; Jackson, Edward R; Browning, Patrick; Huebsch, Wade; Mucino, Victor; Gautam, Mridul. "Flight Simulation of a hybrid projectile to estimate the impact of launch angle on range extension." ASME 2012 International Mechanical Engineering Congress \& Exposition. Houston, 2012. 615-620.

Wilson, Michael J. Projectile Navigation and the Application on Magnetometers. Ann Arbor: ProQuest Information and Learning Company, 2007. Print. 


\section{Appendix AAnalyzing Stability of the M781}

M781 Gyroscopic Stability

$$
S_{g}=\frac{I_{x x}^{2} p^{2}}{2 \rho I_{y y} A_{r e f} d V^{2} C_{m_{\alpha}}}
$$

Where $\mathrm{p}$ is the roll rate in radians per second

$$
\boldsymbol{S}_{\boldsymbol{g}}=\frac{\left(5.4412 \times 10^{-5}\right)^{2}(392.7)^{2}}{2(1.2)\left(1.19215 \times 10^{-4}\right)(0.126)(0.04)\left(76^{2}\right)(1.564)}=\mathbf{3 . 5 1}
$$

Gyroscopically Stable

M781 Dynamic Stability

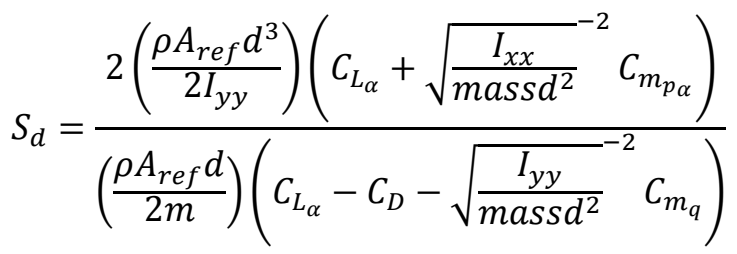

$$
\begin{aligned}
& S_{d}=\frac{2\left(\frac{(1.2)(0.126)\left(.04^{3}\right)}{2\left(1.19215 \times 10^{-4}\right)}\right)\left(2.166+{\sqrt{\frac{5.4412 \times 10^{-5}}{.205\left(.04^{2}\right)}}}^{-2}(-.1325)\right)}{\left(\frac{(1.2)(0.126)(.04)}{2(.205)}\right)\left(2.166-0.2775-{\sqrt{\frac{1.19215 \times 10^{-4}}{.205\left(.04^{2}\right)}}}^{-2}(-5.73)\right)}=6.29
\end{aligned}
$$

Inequality for Dynamic Stability

$$
\begin{gathered}
\frac{1}{S_{g}}>S_{d}\left(2-S_{d}\right) \\
\frac{1}{3.51}>6.29(2-6.29) \\
0.285>-26.98
\end{gathered}
$$

\section{Dynamically Stable}

M781 Static Stability

$$
\begin{gathered}
M=\left(\frac{\rho S d^{3}}{2 I_{y y}}\right) C_{m_{\alpha}} \\
M=\left(\frac{(1.2)(.126) .04^{3}}{2\left(1.19215 \times 10^{-4}\right)}\right) 1.564=0.634
\end{gathered}
$$

Statically Unstable 


\section{Appendix B Hand Calculations for Assumption of Tails Not Affecting Mass Properties}

Calculate the Static Margin (SM) for the baseline $40 \mathrm{~mm}$. Numbers used are cited in text.

$$
\begin{gathered}
\overline{S M}=-\left(\frac{C_{m_{\alpha}}}{C_{L_{\alpha}}}\right)=-\left(\frac{0.0273}{0.0378}\right)=-0.722(\text { Roskam 2003)? } \\
S M=\overline{S M} \ell_{\text {ref }}=-0.722 \times 0.1 \mathrm{~m}=-72.2 \mathrm{~mm}
\end{gathered}
$$

This yields a non-statically stable SM.

$$
S M=X_{A C}-X_{C G}
$$

The CG location for the $40 \mathrm{~mm}$ was found to be $60.41 \%$ back from the nose, meaning:

$$
\begin{gathered}
X_{C G}=\ell_{\text {Total }} 0.6041=86.4 \mathrm{~mm} \text { from nose } \\
\text { where } \ell_{\text {Total }}=\ell_{\text {Tail }}+\frac{1}{2} c_{r}=143 \mathrm{~mm}
\end{gathered}
$$

The AC can be calculated as:

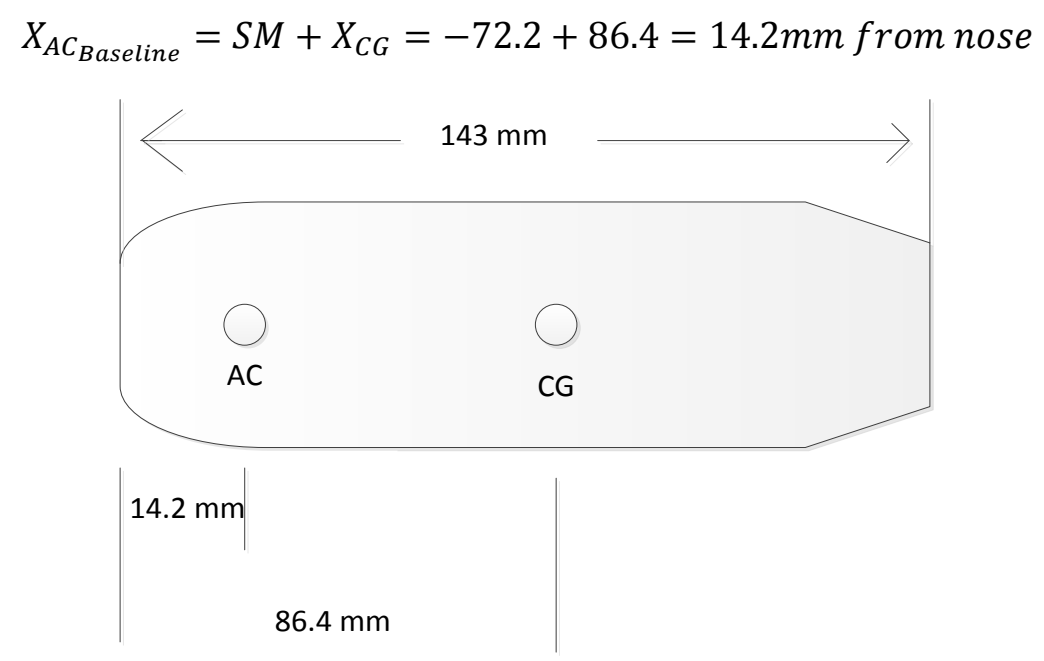

Find the Difference in CG location due to tails being added.

Assumption: Tails are made of Carbon Fiber Sheeting, $1 / 16^{\text {th }}$ inch thick

Density of Carbon Fiber (T300) 1.76g/ $\mathrm{cm}^{3}$ (Torayca 2002)

Total Volume and Mass of Tail Material

$$
\begin{gathered}
\text { Vol }=4(0.159 * 2.0 * 3.0)=3.81 \mathrm{~cm}^{3} \\
\text { Mass }_{\text {Tails }}=\rho_{C F} \mathrm{Vol}=1.76 \times 3.81=6.7 \mathrm{grams}
\end{gathered}
$$

Calculate CG Location with Tails 


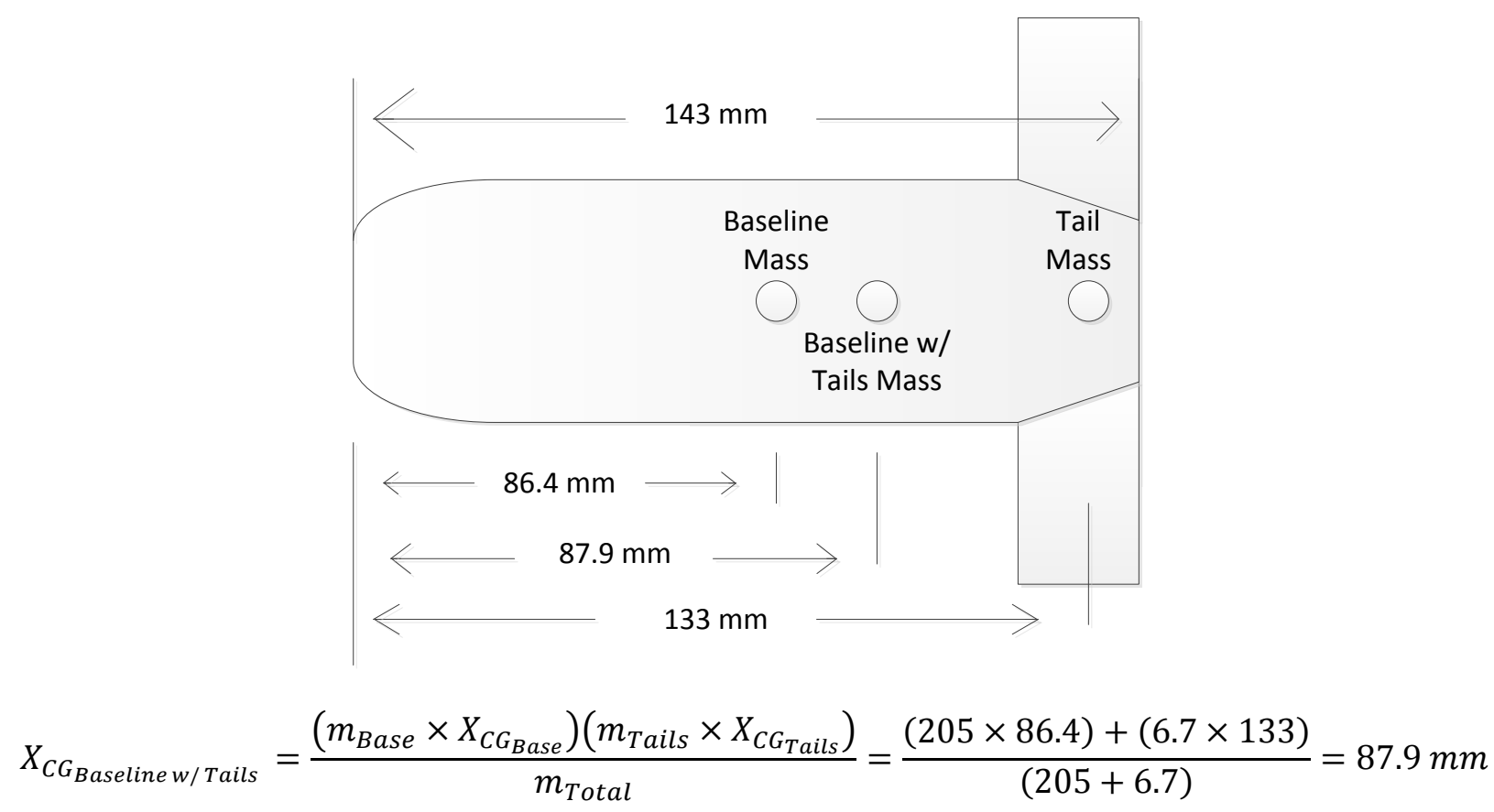

Static Margin for the projectile with Tails

$$
\overline{S M}=-\left(\frac{C_{m_{\alpha}}}{C_{L_{\alpha}}}\right)=-\left(\frac{C_{m_{\alpha_{\text {Body }}}}+C_{m_{\alpha_{\text {Tails }}}}}{C_{L_{\alpha_{\text {Body }}}}+C_{L_{\alpha_{\text {Tails }}}}}\right)=.3196
$$

This yields a -statically stable SM. This was what was also seen from Figure in text.

$$
S M_{\text {Tails }}=.3196 * .1 \mathrm{~m}=31.96 \mathrm{~mm}
$$

Find how much the AC moved by adding tails to the projectile.

$$
X_{A C_{\text {Baseline }} w / \text { Tails }}=S M_{\text {Tails }}+X_{C G_{\text {Tails }}}=32+87.9=119.9 \mathrm{~mm}
$$

The AC shifted $105.7 \mathrm{~mm}$ from adding tails while the CG only moved $1.5 \mathrm{~mm}$. The shift in CG was ignored since its shift was $1.4 \%$ of the magnitude in the AC shift.

Calculate the effect of Tail Mass on the Moment of Inertia.

$$
\begin{gathered}
I_{x x_{M 781}}=5.4412 \times 10^{-5} \mathrm{~kg} \mathrm{~m}^{2} \\
I_{x x_{M 781 \text { w Tails }}}=I_{x x_{M 781}}+m_{\text {Tails }} r_{\text {Tails }}^{2}
\end{gathered}
$$

$\mathrm{r}_{\text {Tails }}$ is the moment arm for the center of mass about the $\mathrm{x}$ axis of the tails.

$$
\begin{gathered}
r_{\text {Tails }}=\frac{\text { dia }}{2}+\frac{\text { span }_{\text {Tails }}}{2}=0.02 \mathrm{~m}+0.015 \mathrm{~m}=0.035 \mathrm{~m} \\
\left.I_{x x_{M 781} \underline{w}_{\text {Tails }}}=5.4412 \times 10^{-5} \mathrm{~kg} \mathrm{~m}^{2}+0.0067 \mathrm{~kg}(0.035 \mathrm{~m})^{2}=6.262 \times 10^{-5} \text { (13\% increase }\right)
\end{gathered}
$$

With the mass of the tails considered the de-spin time was only effected by $2 \%$ of the flight time. So the assumption was made that mass would be ignored. 


\section{Appendix C CG and AC Calculation of Verification Model}

Calculating CG on Test Projectile

\begin{tabular}{|c|c|c|c|}
\hline Component & $\begin{array}{c}\text { Mass } \\
\text { (grams) }\end{array}$ & $\begin{array}{l}\text { Distance From Nose } \\
\text { to CG of component ( } \mathrm{mm} \text { ) }\end{array}$ & Distance x Mass (g mm) \\
\hline Body & 32.7 & 99.5 & 3253.65 \\
\hline Nose & 23.5 & 40.1 & 942.35 \\
\hline Nose Weight & 27 & 35 & 945 \\
\hline Tail Section & 37.2 & 173 & 6435.6 \\
\hline Autopilot & 12 & 99 & 1188 \\
\hline Batteries & 18 & 73.5 & 1323 \\
\hline GPS & 7.6 & 99.5 & 756.2 \\
\hline Voltage Regulator & 6 & 147 & 882 \\
\hline \multirow[t]{3}{*}{ Total } & 164 & & 15725.8 \\
\hline & & $X_{\mathrm{CG}}(\mathrm{mm})$ & 95.88902439 \\
\hline & & $X_{C G}$ (in) & 3.775158441 \\
\hline
\end{tabular}

Calculate Nose Normal Force Coefficient and Center of Pressure

\begin{tabular}{|r|r|l|}
\hline Nose & & \\
\hline Dimensions & & \\
\hline Type & Conical & inches \\
\hline Cone Angle & 15.64225 & deg \\
\hline Normal Force Coeff & & \\
\hline $\mathbf{C}_{\mathbf{N} \alpha \text { Nose }}$ & 2 & \\
\hline Center of Pressure & & \\
\hline $\mathbf{X}_{\mathbf{N}}$ & 2 & \\
\hline
\end{tabular}

$$
\begin{gathered}
C_{N_{\alpha \text { Nose }}}=2 \text {, for all nose shape } \\
X_{\text {Nose }}=\left(\frac{2}{3}\right) L_{\text {Nose }}
\end{gathered}
$$

... Other Nose Shapes have a different multiplying factor, found in (Barrowman n.d.). 
Body Normal Force Coefficient

\begin{tabular}{|c|c|c|c|}
\hline Cylindrical Body & & & \\
\hline \multicolumn{4}{|l|}{ Dimensions } \\
\hline Length & 3.629921 & inches & \\
\hline Diameter & 1.68 & inches & \\
\hline Inner Diameter & 1.44378 & inches & \\
\hline \multicolumn{4}{|l|}{ Normal Force Coeff } \\
\hline $\mathrm{C}_{\mathrm{N} \alpha \text { Body }}$ & 0 & (At sma & all AoA, <10deg) \\
\hline
\end{tabular}

Calculate Boat Tail Normal Force Coefficient and Center of Pressure

$$
\begin{aligned}
& \begin{array}{|l|l|l|}
\hline \text { Boat Tail } & & \\
\hline \text { Dimensions } & & \\
\hline \text { Length } & 2 & \text { inches } \\
\hline
\end{array} \\
& \text { Front Diameter } 1.68 \text { inches } \\
& \begin{array}{lr|r|}
\hline \text { Rear Diameter } & 0.5 \text { inches } \\
\hline
\end{array} \\
& \begin{array}{l|l}
\text { Cone Angle } & 16.43606 \mathrm{deg} \\
\hline
\end{array} \\
& \begin{array}{l|l|}
\text { Length of Full Cone } 3.006855 & \text { inches } \\
\hline
\end{array} \\
& \text { Normal Force Coeff } \\
& \begin{array}{|r|r|l|}
\hline \mathrm{C}_{\mathrm{N} \alpha \mathrm{BT}} & -1.82285 & \\
\hline \text { Center of Pressure } & & \\
\hline \mathrm{X}_{\mathrm{BT}} & 7.449493 & \text { inches } \\
\hline
\end{array} \\
& \left.C_{N_{\alpha \text { Boat Tail }}}=2\left[\left(\frac{\text { dia }_{\text {Rear }}}{\text { dia }}\right)_{\text {Body }}\right)^{2}-\left(\frac{\text { dia }_{\text {Front }}}{\text { dia }_{\text {Body }}}\right)^{2}\right] \\
& X_{\text {Boat Tail }}=L_{\text {Nose }}+L_{\text {Body }}+\frac{L_{\text {Boat Tail }}}{3}\left[1+\frac{1-\frac{\text { dia }_{\text {Front }}}{\text { dia }_{\text {Rear }}}}{1-\left(\frac{\text { dia }_{\text {Front }}}{\text { dia }_{\text {Rear }}}\right)^{2}}\right]
\end{aligned}
$$

Given Cylindrical Tail Normal Force Coefficient

\begin{tabular}{|r|r|l|l|}
\hline Cylindrical Body (Tail) & & \\
\hline Length & 2 & inches & \\
\hline Diameter & 0.5 & inches & \\
\hline Normal Force Coeff & & & \\
\hline $\mathrm{C}_{\mathrm{N} \alpha \text { Body }}$ & \multicolumn{3}{|l|}{0} \\
(At small AoA, <10deg) \\
\hline
\end{tabular}


Calculate Fins Normal Force Coefficient and Center of Pressure

\begin{tabular}{|r|r|l|}
\hline Fins & & \\
\hline Dimensions & & \\
\hline m & -2 & inches \\
\hline tip chord & 4 & inches \\
\hline root chord & 2 & inches \\
\hline span & 0.599213 & inches \\
\hline Number of Tails & 4 & \\
\hline Length & 1.165785 & inches \\
\hline Normal Force Coeff & & \\
\hline $\mathrm{C}_{\mathrm{N} \alpha \text { Tail }}$ & 7.174792 & \\
\hline Center of Pressure & & \\
\hline $\mathrm{X}_{\text {fins }}$ & 8.296588 & inches \\
\hline
\end{tabular}

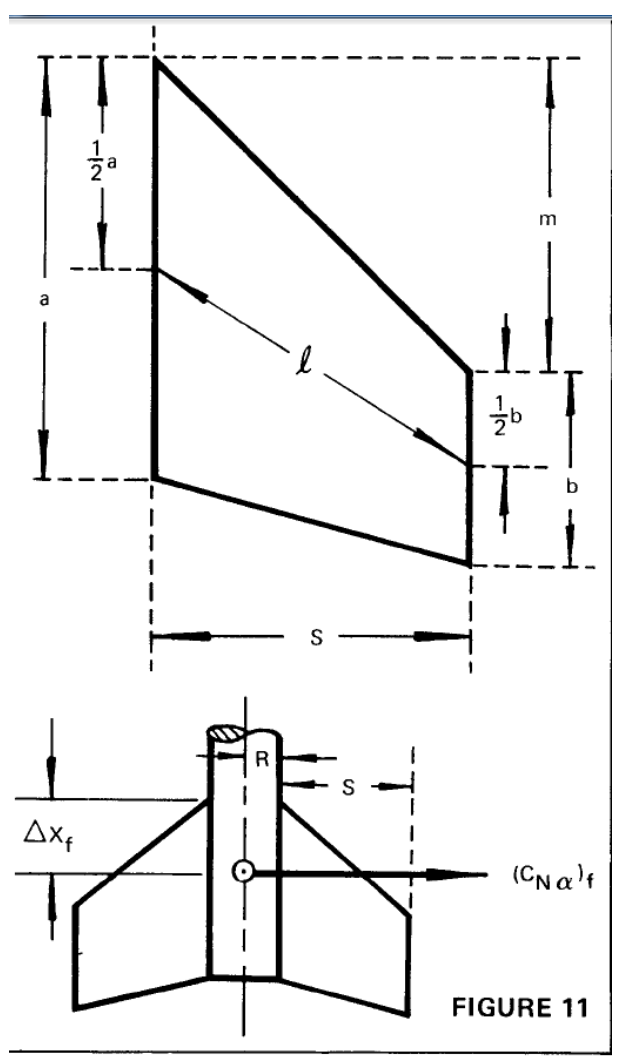

Figure C-1: Sketch to Determine Tail Dimensions (Barrowman n.d.)

$$
\begin{aligned}
& C_{N_{\alpha \text { Fin }}}=\frac{\left.4 N\left(\frac{\text { span }}{\text { dia }}\right)_{\text {Body }}\right)^{2}}{1+\sqrt{1+\left(\frac{2 l}{a+b}\right)^{2}}} \\
& X_{\text {Fin }}=L_{\text {Nose }}+L_{\text {Body }}+L_{\text {Boat Tail }}+\frac{m(a+2 b)}{3(a+b)}+\frac{1}{6}\left(a+b-\frac{a b}{a+b}\right)
\end{aligned}
$$




\section{Appendix D Aerodynamic Build-up for Test Projectile}

\section{Lift Coefficient calculation Test Projectile}

$$
C_{L_{\alpha}} \approx C_{N_{\alpha}}=7.35 \frac{\pi}{180}=\mathbf{0 . 1 2 8}
$$

Pitching Moment Coefficient calculation Test Projectile

$$
\begin{gathered}
S M=\left(X_{A C}-X_{C G}\right)=(6.79 \text { in }-3.78 \text { in }) \times\left(\frac{0.0254 m}{1 \text { in }}\right)=0.076454 m \\
\overline{S M}=\frac{S M}{\ell_{\text {ref }}}=\frac{0.076454 m}{0.1 m}=0.76454 m \\
C_{\boldsymbol{m}_{\alpha}}=-\overline{S M} C_{N_{\alpha}}=-0.76454 \times 0.128=-\mathbf{0 . 0 9 8} \ldots(\text { Stable })(\text { Roskam 2003) }
\end{gathered}
$$

Drag Coefficient calculation Test Projectile (Gregorek 1970)

$$
\begin{gathered}
C_{D_{\text {Total }}}=C_{D_{\text {Nose }}}+C_{D_{\text {Base }}}+C_{D_{\text {Body }}}+C_{D_{\text {Tail }}} \\
\left(\frac{\text { Length }}{\text { Diameter }}\right)_{\text {Test Projectile }}=\frac{9.12 \text { in }}{1.68 \text { in }}=5.43(\text { Needed for Fig. 28)(Gregorek 1970) } \\
C_{D_{\text {Nose }}}+C_{D_{\text {Body }}}=0.12(\text { Fig. 28) } \\
C_{D_{\text {Tail }}}=.04(\text { Fig. 38) } \\
C_{D_{\text {Base }}}=\frac{0.029}{\sqrt{C_{D_{\text {Nose }}}+C_{D_{\text {Body }}}}}=\frac{0.029}{\sqrt{0.12}}=.08(\text { Eq. 9) (Gregorek 1970)? } \\
C_{D_{\text {Total }}}=0.12+0.04+0.08=\mathbf{0 . 2 4}
\end{gathered}
$$




\section{Appendix E Px4 Autopilot Setup}

The Px4 Autopilot/Flight Management Unit was used as the autopilot to determine the attitude, location and velocity of the projectile as well as be used to deflect the control surfaces (PX4 Autopilot n.d.). A GPS antenna was connected to the autopilot. This autopilot was activated in standalone mode, meaning no input other than power was required to begin collecting data. A SD card holds the code required to do this as well as it holds the flight data to be viewed, at a later time. The process that was required, to load code on the SD card, was as follows:

1. Download Python Version 3.2.2

2. Make a folder in the SD card labeled "etc"

a. Create a file named "rc"

i. In this file using word pad create a command script

attitude est ekf start

mpu6000 start

hmc5883 start

ms5611 start

segway start -p mpu6000

sdlog2 start -r 100 -e

3. To run "rc" on autopilot connect autopilot to power with SD card inserted

4. When the GPS LED stops flashing the autopilot is finally receiving GPS data to log

5. Disconnect from power after flight is completed

6. Remove SD card from autopilot and insert into SD reader

7. Open last .sess folder to get to the log file

8. To reduce data from the log, copy the log file and Matlab script to the same location in python

9. Run Matlab script to create an excel data file and plot the results 


\section{Appendix F Calculate Estimated Delta Distance Sub-system}

function delta_dist=

FlightAngle(current_distance,current_altitude,desired_distance,desired_altitu de, x_vel,z_vel, current_pitch)

$\mathrm{CL}==\%$ (Vector of lift coeff. from tail un-deflection);

$C D==\%$ (Vector of drag coeff. from tail un-deflection);

$\mathrm{Vx}=\mathrm{x} \_\mathrm{vel}$;

$\mathrm{Vz}=-\mathrm{z} \_\mathrm{vel}$;

Pitch=current_pitch;

Alpha $=\%$ (Vector of AoA from tail un-deflection);

$z=0$;

$\mathrm{x}=\mathrm{\theta}$;

rho=1.2;

$\mathrm{A}=\mathrm{pi}^{*} .02^{\wedge} 2$;

mass $=.19937$;

$\mathrm{g}=9.81$;

V_total=sqrt $\left(\mathrm{Vx}^{\wedge} 2+\mathrm{V} z^{\wedge} 2\right)$;

final_altitude=-current_altitude ;

final_distance $=0$;

time $=0$;

$i=1$;

while final_altitude>=desired_altitude delta_t=.005;

time=time+delta_t;

$A x=\left(\right.$ sind $($ abs $(P i t c h)){ }^{*} C L(i) * .5 *$ rho*V_total^ $2{ }^{*} A-$

$\operatorname{cosd}(\operatorname{abs}(P i t c h)){ }^{*} C D(i) * \cdot 5^{*}$ rho*V_total^ $\left.2^{\star} A\right) /$ mass;

$\mathrm{Az}=-$

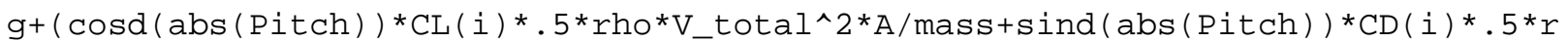

ho*V_total^2*A)/mass;

$V z=V z+A z *$ delta_t;

$\mathrm{Vx}=\mathrm{Vx}+\mathrm{Ax} \mathrm{x}^{*}$ delta_t;

$z=z+V z^{*}$ delta_t;

$\mathrm{x}=\mathrm{x}+\mathrm{Vx}{ }^{*}$ delta_t;

Pitch=atand $(V z / V x)+A l p h a(i)$;

final_distance $=x+$ current_distance ;

final_altitude $=z$-current_altitude;

V_total=sqrt $\left(V x^{\wedge} 2+V z^{\wedge} 2\right)$;

$i=i+1$;

if i>length $(\mathrm{CL})$

end $i=$ length $(\mathrm{CL})$;

end

delta_dist=desired_distance-final_distance; 


\section{Appendix G Projectile Motion with Drag}

The following equations were used to estimate the distance and altitude of the projectile to compare with results from the roll controller section. In these equations all variables will remain the same except for the drag coefficient. By adding tails to the projectile the drag coefficient was increased. The drag coefficient of the projectile by itself is 0.2775 and becomes 0.2982 with tails added. Results of each trajectory are shown in the following figure. Using the equations the range was calculated to decrease $1.9 \%$ and the maximum altitude was seen to be reduced by $1.1 \%$.

$$
\begin{gathered}
V_{t}=\sqrt{\frac{2 M_{p} g}{C_{d} A_{\text {ref }} \rho}} \\
V_{y}=V_{t} \frac{V_{y_{0}}-V_{t} \tan \left(t g / V_{t}\right)}{V_{t}-V_{o} \tan \left(t g / V_{t}\right)} \\
V_{x}=\frac{V_{t}^{2} * V_{x_{0}}}{V_{t}^{2}+g V_{x_{0}} t} \\
y=\frac{V_{t}^{2}}{2 g} \ln \left(\frac{V_{0}^{2}+V_{t}^{2}}{V_{y}^{2}+V_{t}^{2}}\right) \\
x=\frac{V_{t}^{2}}{g} \ln \left(\frac{V_{t}^{2}+g V_{x_{0}} t}{V_{t}^{2}}\right)
\end{gathered}
$$

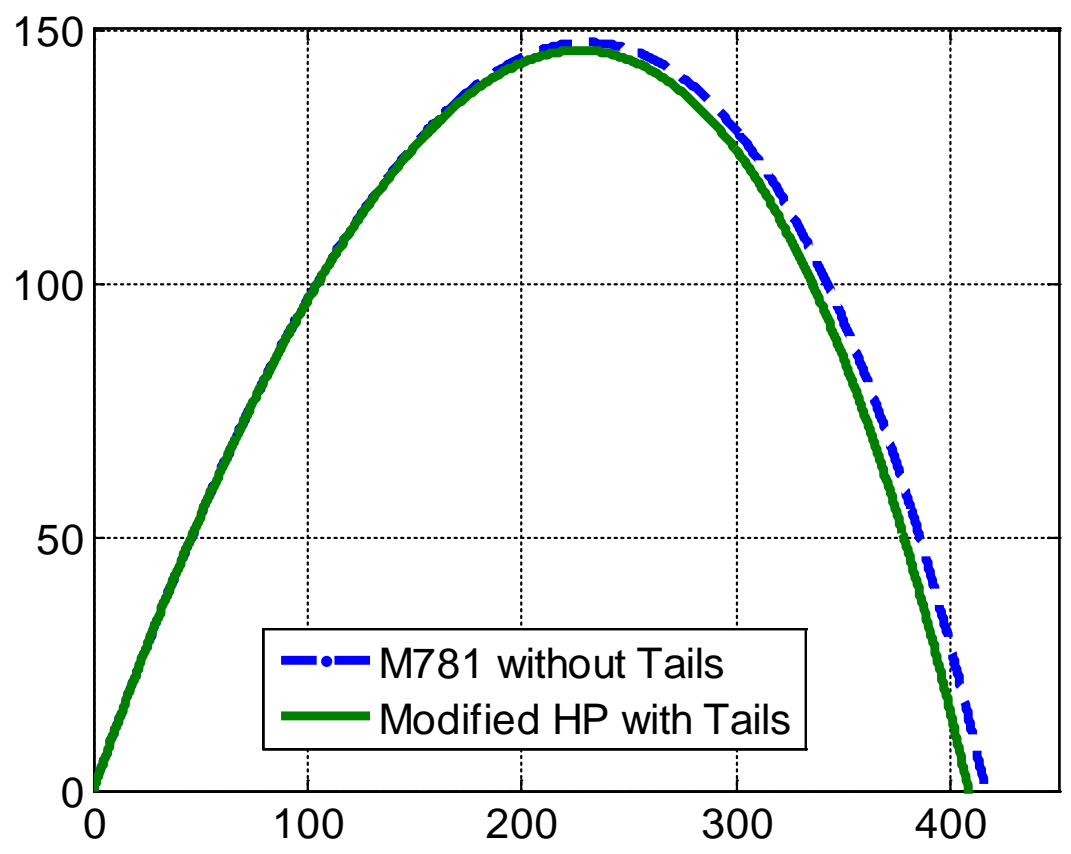

